\title{
PERIODS AND MOTIVES IN THE SPECTRAL ACTION OF ROBERTSON-WALKER SPACETIMES
}

\author{
FARZAD FATHIZADEH AND MATILDE MARCOLLI
}

\begin{abstract}
We show that, when considering the scaling factor as an affine variable, the coefficients of the asymptotic expansion of the spectral action on a (Euclidean) Robertson-Walker spacetime are periods of mixed Tate motives, involving relative motives of complements of unions of hyperplanes and quadric hypersurfaces and divisors given by unions of coordinate hyperplanes.
\end{abstract}

\section{Contents}

1. Introduction

1.1. The spectral model of gravity

2. Robertson-Walker metric and the Dirac operator

2.1. Pseudodifferential symbol

3. Heat expansion and the Wodzicki residue

4. The $a_{2}$ term and quadric surfaces in $\mathbb{P}^{3}$

5. The $a_{4}$ term and quadric hypersurfaces in $\mathbb{P}^{5}$

6. The $a_{2 n}$ term

7. The motives

7.1. Pencils of quadrics

1

7.2. Motives of quadrics

7.3. Grothendieck classes

7.4. Pencils of quadrics in $\mathbb{P}^{3}$

7.5. Pencils of quadrics in $\mathbb{P}^{5}$

7.6. The Grothendieck class of $\mathbb{P}^{2 n-1} \backslash Z_{\alpha, 2 n}$ over $\mathbb{K}=\mathbb{Q}(\sqrt{-1})$

7.7. The motive of $Z_{\alpha, 2 n}$ over $\mathbb{Q}$

Appendix A: Full expression for $b_{-6} \quad 33$

Acknowledgement

References

\section{InTRODUCTION}

Over the past decade, Grothendieck's theory of motives has come to play an increasingly important role in theoretical physics. While the existence of a relation between motives and periods of algebraic varieties and computations in high-energy physics might have seemed surprising and unexpected, the existence of underlying 
motivic structures in quantum field theory has now been widely established, see for instance [2], 3], [7], [14]. Typically, periods and motives occur in quantum field theory in the perturbative approach, through the asymptotic expansion in Feynman diagrams, where in the terms of the asymptotic expansion the renormalized Feynman integrals are identified with periods of certain hypersurface complements. The nature of the motive of the hypersurface constraints the class of numbers that can occur as periods. Similarly, a large body of recent work on amplitudes in $N=4$ Supersymmetric Yang-Mills has uncovered another setting where the connection to periods and motives plays an important role, see [1], [11, [12].

In this paper, we present another surprising instance of the occurrences of periods and motives in theoretical physics, this time in a model of (modified) gravity based on the spectral action functional of [5]. The situation is somewhat similar to the one seen in the quantum field theory setting, with some important differences. As in the QFT framework, we deal with an asymptotic expansion, which in our case is given by the large energy expansion of the spectral action functional. We show in this paper that, in the case of (Euclidean) Robertson-Walker spacetimes, the terms of the asymptotic expansion of the spectral action functional can be expressed as periods of mixed Tate motives, given by complements of quadric hypersurfaces. An important difference, with respect to the case of a scalar massless quantum field theory of [2], is that here we need to consider only one quadric hypersurface for each term of the expansion, whereas in the quantum field theory case one has to deal with the much more complicated motive of a union of quadric hypersurfaces, associated to the edges of the Feynman graph. On the other hand, the algebraic differential form that is integrated on a semi-algebraic set in the hypersurface complement is much more complicated in the spectral action case considered here, than in the quantum field theory case: the terms in the algebraic differential form arise from the computation, via pseudo-differential calculus, of a parametrix for the square of the Dirac operator on the Robertson-Walker spacetime, after a suitable change of variables in the integral. While the explicit expression of the differential form, even for the simplest cases of the coefficients $a_{2}$ and $a_{4}$ can take up several pages, the structure of the terms can be understood, as we explain in the following sections, and the domain of definition is, in the case of the $a_{2 n}$ term, the complement of a union of two hyperplanes and a quadric hypersurfaces defined by a family of quadrics $Q_{\alpha, 2 n}$ in an affine space $\mathbb{A}^{2 n+3}$.

In Section 2 we describe our choice of coordinates, and the resulting form of the pseudodifferential symbol of the square of the Dirac operator on a (Euclidean) Robertson-Walker metric. In Section 3, we describe briefly how the Seeley-DeWitt coefficients of the heat kernel expansion can be computed in terms of Wodzicki residues, by taking products with auxiliary tori with flat metrics. Section 4 gives the explicit computation of the $a_{2}$ term, showing that, before integrating in the time variable, and treating the scaling factor as an affine parameter $\alpha \in \mathbb{A}^{1} \backslash\{0\}=\mathbb{G}_{m}$, one can write the resulting integral as a period obtained by integrating an algebraic (over $\mathbb{Q}$ ) differential form over a $\mathbb{Q}$-semi-algebraic set. The differential form is defined on the complement in $\mathbb{A}^{5}$ of a union of two hyperplanes and the quadric determined by the 
vanishing of the quadratic form $Q_{\alpha, 2}=u_{1}^{2}+\alpha^{-2}\left(u_{2}^{2}+u_{3}^{2}+u_{4}^{2}\right)$. The $\mathbb{Q}$-semialgebraic set in this hypersurface complement has boundary contained in a divisor given by a union of coordinate hyperplanes. Although the boundary divisor and the hypersurface intersect nontrivially, all the integrals are convergent and we do not have a renormalization problem, unlike what happens in the quantum field theory setting. In Section 5 we prove an analogous result for the $a_{4}$ term, with the very lengthy full expression of the $a_{4}$ term given in the appendix. In Section 6, using an inductive argument and the result of the previous cases, we prove that the terms $a_{2 n}$ can all be identified (prior to time-integration) with periods of motives of complements of quadric hypersurfaces obtained from a family of quadrics

$$
Q_{\alpha, 2 n}=u_{1}^{2}+\alpha^{-2}\left(u_{2}^{2}+u_{3}^{2}+u_{4}^{2}\right)+u_{5}^{2}+\cdots+u_{2 n+2}^{2} .
$$

The algebraic differential forms depend on $2 n$ auxiliary affine parameters $\alpha_{1}, \ldots, \alpha_{2 n}$, which correspond to the time derivatives of the scaling factor of the RobertsonWalker metric. In Section 7 we analyze more explicitly the motive, showing that, over a quadratic field extension $\mathbb{Q}(\sqrt{-1})$ where the quadrics become isotropic, it is a mixed Tate motive, while over $\mathbb{Q}$ it is a form of a Tate motive in the sense of [16], [18], [19]. We compute explicitly, by a simple inductive argument, the class in the Grothendieck ring of the relevant hypersurface complement.

1.1. The spectral model of gravity. The spectral action functional, introduced in [5] is a regularized trace of the Dirac operator $D$ given by

$$
\mathcal{S}(\Lambda)=\operatorname{Tr}(f(D / \Lambda))=\sum_{\lambda \in \operatorname{Spec}(D)} \operatorname{Mult}(\lambda) f(\lambda / \Lambda),
$$

where the test function $f$ is a smooth even rapidly decaying function, which should be thought of as a smooth approximation to a cutoff function. The parameter $\Lambda>0$ is an energy scale. One of the main advantages of this action functional is that it is not only defined for smooth compact Riemannian spin manifolds, but also for a more general class of geometric objects that include the noncommutative analogs of Riemannian manifolds, finitely summable spectral triples, see [6]. In particular, the spectral action functional applied to almost commutative geometries (products of manifolds and finite noncommutative spaces) is used as a method to generate particle physics models with varying possible matter sectors depending on the finite geometry and with matter coupled to gravity, see [17] for a recent overview. It was shown in [5] that, in the case of commutative and almost commutative geometries, the spectral action functional has an asymptotic expansion for large energy $\Lambda$,

$$
\operatorname{Tr}(f(D / \Lambda)) \sim \sum_{\beta \in \Sigma_{S T}^{+}} f_{\beta} \Lambda^{\beta} f|D|^{-\beta}+f(0) \zeta_{D}(0)+\cdots,
$$

where the coefficients depend on momenta $f_{\beta}=\int_{0}^{\infty} f(v) v^{\beta-1} d v$ and Taylor coefficients of the test function $f$ and on residues

$$
f|D|^{-\beta}=\frac{1}{2} \operatorname{Res}_{s=\beta} \zeta_{D}(s)
$$


at poles of the zeta function $\zeta_{D}(s)$ of the Dirac operator. The leading terms of the asymptotic expansion recover the usual local terms of an action functional for gravity, the Einstein-Hilbert action with cosmological term, with additional modified gravity terms given by Weyl conformal gravity and Gauss-Bonnet gravity. In the case of an almost commutative geometry the leading terms of the asymptotic expansion also determine the Lagrangian of the resulting particle physics model. The spectral action on ordinary manifold, as an action functional of modified gravity, was applied to cosmological models, see [15] for an overview. In the manifold case, the Mellin transform relation between zeta function and trace of the heat kernel expresses the coefficients of the spectral action expansion in terms of the Seeley-DeWitt coefficients $a_{2 n}$ of the heat kernel expansion,

$$
\operatorname{Tr}\left(e^{-t D^{2}}\right) \sim_{t \rightarrow 0+} \quad t^{-m / 2} \sum_{n=0}^{\infty} a_{2 n}\left(D^{2}\right) t^{n} .
$$

Pseudodifferential calculus techniques and the parametrix method can then be applied to the computation of the symbol and the Seeley-DeWitt coefficients. The resulting computations can easily become intractable, but a computationally more efficient method introduced in [8], based on Wodzicki residues and products by auxiliary flat tori can be applied to make the problem more easily tractable.

In the case of the (Euclidean) Robertson-Walker spacetimes, it was conjectured in [4] and proved in [10] that all the terms in the expansion of the spectral action are polynomials with rational coefficients in the scaling factor and its derivatives. This rationality result suggests the existence of an underlying arithmetic structure. In the case of the Bianchi IX metrics, a similar rationality result was proved in [8] and the underlying arithmetic structure was analyzed in [9] for the Bianchi IX gravitational instantons, in terms of modular forms. Here we consider the case of the RobertsonWalker spacetimes and we look for arithmetic structures in the expansion of the spectral action in terms of periods and motives. A similar motivic analysis of the Bianchi IX case will be carried out in forthcoming work.

\section{Robertson-Walker metric and the Dirac operator}

We consider the Robertson-Walker metric with the expansion factor $a(t)$,

$$
d s^{2}=d t^{2}+a(t)^{2} d \sigma^{2},
$$

where $d \sigma^{2}$ is the round metric on the 3-dimensional sphere $\mathbb{S}^{3}$. Using the Hopf coordinates for $\mathbb{S}^{3}$, we use the local chart

$$
\begin{gathered}
x=\left(t, \eta, \phi_{1}, \phi_{2}\right) \mapsto\left(t, \sin \eta \cos \phi_{1}, \sin \eta \sin \phi_{2}, \cos \eta \cos \phi_{1}, \cos \eta \sin \phi_{2}\right), \\
0<\eta<\frac{\pi}{2}, \quad 0<\phi_{1}<2 \pi, \quad 0<\phi_{2}<2 \pi .
\end{gathered}
$$

In this coordinate system, the Robertson-Walker metric is written as

$$
d s^{2}=d t^{2}+a(t)^{2}\left(d \eta^{2}+\sin ^{2}(\eta) d \phi_{1}^{2}+\cos ^{2}(\eta) d \phi_{2}^{2}\right)
$$


or alternatively we write:

$$
\left(g_{\mu \nu}\right)=\left(\begin{array}{cccc}
1 & 0 & 0 & 0 \\
0 & a(t)^{2} & 0 & 0 \\
0 & 0 & a(t)^{2} \sin ^{2}(\eta) & 0 \\
0 & 0 & 0 & a(t)^{2} \cos ^{2}(\eta)
\end{array}\right)
$$

with

$$
\left(g^{\mu \nu}\right)=\left(g_{\mu \nu}\right)^{-1}=\left(\begin{array}{cccc}
1 & 0 & 0 & 0 \\
0 & \frac{1}{a(t)^{2}} & 0 & 0 \\
0 & 0 & \frac{\csc ^{2}(\eta)}{a(t)^{2}} & 0 \\
0 & 0 & 0 & \frac{\sec ^{2}(\eta)}{a(t)^{2}}
\end{array}\right)
$$

2.1. Pseudodifferential symbol. One can write the local expression for the Dirac operator $D$, as in $\S 2$ of [10], and one finds that the pseudodifferential symbol $\sigma_{D}(x, \xi)$ of $\mathrm{D}$ is given by the sum $q_{1}(x, \xi)+q_{0}(x, \xi)$, where, using the notation $\xi=\left(\xi_{1}, \xi_{2}, \xi_{3}, \xi_{4}\right) \in$ $\mathbb{R}^{4}$ for an element of the cotangent fibre $T_{x}^{*} M \simeq \mathbb{R}^{4}$ at the point $x=\left(t, \eta, \phi_{1}, \phi_{2}\right)$, we have

$$
\begin{gathered}
q_{1}(x, \xi)=\left(\begin{array}{cccc}
0 & 0 & \frac{i \sec (\eta) \xi_{4}}{a(t)}-\xi_{1} & \frac{i \xi_{2}}{a(t)}+\frac{\csc (\eta) \xi_{3}}{a(t)} \\
0 & 0 & \frac{i \xi_{2}}{a(t)}-\frac{\csc (\eta) \xi_{3}}{a(t)} & -\xi_{1}-\frac{i \sec (\eta) \xi_{4}}{a(t)} \\
-\xi_{1}-\frac{i \sec (\eta) \xi_{4}}{a(t)} & -\frac{i \xi_{2}}{a(t)}-\frac{\csc (\eta) \xi_{3}}{a(t)} & 0 & 0 \\
\frac{\csc (\eta) \xi_{3}}{a(t)}-\frac{i \xi_{2}}{a(t)} & \frac{i \sec (\eta) \xi_{4}}{a(t)}-\xi_{1} & 0 & 0
\end{array}\right), \\
q_{0}(\xi)=\left(\begin{array}{cccc}
0 & 0 & \frac{3 i a^{\prime}(t)}{2 a(t)} & \frac{\cot (\eta)-\tan (\eta)}{2 a(t)} \\
0 & 0 & \frac{\cot (\eta)-\tan (\eta)}{2 a(t)} & \frac{3 i a^{\prime}(t)}{2 a(t)} \\
\frac{3 i a^{\prime}(t)}{2 a(t)} & \frac{\tan (\eta)-\cot (\eta)}{2 a(t)} & 0 & 0
\end{array}\right) .
\end{gathered}
$$

These matrices can be used to find the pseudodifferential symbol of the square of the Dirac operator:

$$
\sigma_{D^{2}}(x, \xi)=p_{2}(x, \xi)+p_{1}(x, \xi)+p_{0}(x, \xi),
$$

where, denoting the $4 \times 4$ identity matrix by $I_{4 \times 4}$, we have:

$$
\begin{gathered}
p_{2}(x, \xi)=q_{1}(x, \xi) q_{1}(x, \xi)=\left(\sum g^{\mu \nu} \xi_{\mu} \xi_{\nu}\right) I_{4 \times 4} \\
=\left(\xi_{1}^{2}+\frac{\xi_{2}^{2}}{a(t)^{2}}+\frac{\csc ^{2}(\eta) \xi_{3}^{2}}{a(t)^{2}}+\frac{\sec ^{2}(\eta) \xi_{4}^{2}}{a(t)^{2}}\right) I_{4 \times 4}, \\
p_{1}(x, \xi)=q_{0}(x, \xi) q_{1}(x, \xi)+q_{1}(x, \xi) q_{0}(x, \xi)+\sum_{j=1}^{4}-i \frac{\partial q_{1}}{\partial \xi_{j}}(x, \xi) \frac{\partial q_{1}}{\partial x_{j}}(x, \xi),
\end{gathered}
$$




$$
p_{0}(x, \xi)=q_{0}(x, \xi) q_{0}(x, \xi)+\sum_{j=1}^{4}-i \frac{\partial q_{1}}{\partial \xi_{j}}(x, \xi) \frac{\partial q_{0}}{\partial x_{j}}(x, \xi)
$$

\section{HeAt eXPANSiOn And the Wodzicki RESIDUE}

It is in general computationally difficult to obtain explicit expressions for the Seeley-DeWitt coefficients of the heat kernel expansions, even for nicely homogeneous and isotropic metrics like the Friedmann-Robertson-Walker case. A computationally more efficient method was introduced in [8], based on products with auxiliary flat tori and Wodzicki residues. We apply it here to calculate the coefficients $a_{2 n}$ that appear in the small time heat kernel expansion

$$
\operatorname{Trace}\left(e^{-s D^{2}}\right) \sim_{s \rightarrow 0^{+}} a_{0} s^{-2}+a_{2} s^{-1}+a_{4}+a_{6} s+a_{8} s^{2}+\cdots .
$$

In fact, it is proved in [8] that, for any non-negative even integer $r$, we have

$$
a_{2+r}=\frac{1}{2^{5} \pi^{4+r / 2}} \operatorname{Res}\left(\Delta^{-1}\right)
$$

where

$$
\Delta=D^{2} \otimes 1+1 \otimes \Delta_{\mathbb{T}^{r}}
$$

in which $\Delta_{\mathbb{T}^{r}}$ is the flat Laplacian on the $r$-dimensional torus $\mathbb{T}^{r}=(\mathbb{R} / \mathbb{Z})^{r}$. Here, the linear functional Res defined on the algebra of classical pseudodifferential operators is the Wodzicki residue, which is defined as follows. Assume that the dimension of the manifold is $m$, and that the symbol of a classical pseudodifferential operator is given in a local chart $U$ by

$$
\sigma(x, \xi) \sim \sum_{j=0}^{\infty} \sigma_{d-j}(x, \xi) \quad(\xi \rightarrow \infty)
$$

where each $\sigma_{d-j}: U \times\left(\mathbb{R}^{m} \backslash\{0\}\right) \rightarrow M_{r}(\mathbb{C})$ is positively homogeneous of order $d-j$ in $\xi$. Then one needs to consider the 1-density defined by

$$
\operatorname{wres}_{x} P_{\sigma}=\left(\int_{|\xi|=1} \operatorname{tr}\left(\sigma_{-m}(x, \xi)\right)\left|\sigma_{\xi, m-1}\right|\right)\left|d x^{0} \wedge d x^{1} \wedge \cdots \wedge d x^{m-1}\right|,
$$

in which $\sigma_{\xi, m-1}$ is the volume form of the unit sphere $|\xi|=1$ in the cotangent fibre $\mathbb{R}^{m} \simeq T_{x}^{*} M:$

$$
\sigma_{\xi, m-1}=\sum_{j=1}^{m}(-1)^{j-1} \xi_{j} d \xi_{1} \wedge \cdots \wedge \widehat{d}_{j} \wedge \cdots \wedge d \xi_{m}
$$

The Wodzicki residue of the pseudodifferential operator $P_{\sigma}$ associated with the symbol $\sigma$ is by definition the integral of the above 1-density associated to $\sigma$ :

$$
\operatorname{Res}\left(P_{\sigma}\right)=\int_{M} \operatorname{wres}_{x} P_{\sigma} \text {. }
$$

One can find a detailed discussion of the Wodzicki residue in Chapter 7 of [13] and references therein. 


\section{THE $a_{2}$ TERM AND QUADRIC SURFACES IN $\mathbb{P}^{3}$}

We will refer here to the form of the $a_{2}$ term before performing the integration in the time variable $t$. As we show below, this is the part that is naturally expressible in terms of periods of motives.

Theorem 4.1. The form $b_{-4}(x, \xi)$ derived from $\operatorname{tr}\left(\sigma_{-4}(x, \xi)\right)$ computing the $a_{2}$ term of the heat kernel expansion of $D^{2}$ is a rational differential form

$$
\Omega=f \widetilde{\sigma}_{3},
$$

in affine coordinates $\left(u_{0}, u_{1}, u_{2}, u_{3}, u_{4}\right) \in \mathbb{A}^{5}, \alpha \in \mathbb{G}_{m}$, and $\left(\alpha_{1}, \alpha_{2}\right) \in \mathbb{A}^{2}$ where the functions

$$
f\left(u_{0}, u_{1}, u_{2}, u_{3}, u_{4}, \alpha, \alpha_{1}, \alpha_{2}\right)=f_{\left(\alpha_{1}, \alpha_{2}\right)}\left(u_{0}, u_{1}, u_{2}, u_{3}, u_{4}, \alpha\right)
$$

are $\mathbb{Q}$-linear combinations of rational functions of the form

$$
\frac{P\left(u_{0}, u_{1}, u_{2}, u_{3}, u_{4}, \alpha, \alpha_{1}, \alpha_{2}\right)}{\alpha^{2 r} u_{0}^{k}\left(1-u_{0}\right)^{m}\left(u_{1}^{2}+\alpha^{-2}\left(u_{2}^{2}+u_{3}^{2}+u_{4}^{2}\right)\right)^{\ell}},
$$

where

$$
P\left(u_{0}, u_{1}, u_{2}, u_{3}, u_{4}, \alpha, \alpha_{1}, \alpha_{2}\right)=P_{\left(\alpha_{1}, \alpha_{2}\right)}\left(u_{0}, u_{1}, u_{2}, u_{3}, u_{4}, \alpha\right)
$$

are polynomials in $\mathbb{Q}\left[u_{0}, u_{1}, u_{2}, u_{3}, u_{4}, \alpha, \alpha_{1}, \alpha_{2}\right]$ and where $r, k, m$ and $\ell$ are nonnegative integers, and with $\widetilde{\sigma}_{3}=\widetilde{\sigma}_{3}\left(u_{0}, u_{1}, u_{2}, u_{3}, u_{4}\right)$ the algebraic differential form

$\frac{1}{2}\left(u_{1} d u_{0} d u_{2} d u_{3} d u_{4}-u_{2} d u_{0} d u_{1} d u_{3} d u_{4}+u_{3} d u_{0} d u_{1} d u_{2} d u_{4}-u_{4} d u_{0} d u_{1} d u_{2} d u_{3}\right)$.

The forms $\Omega^{\alpha}=\Omega_{\left(\alpha_{1}, \alpha_{2}\right)}^{\alpha}$ obtained by restricting the above to a fixed value of $\alpha \in$ $\mathbb{A}^{1} \backslash\{0\}$ are a two parameter family of algebraic differential forms on the algebraic variety over $\mathbb{Q}$ given by the complement in $\mathbb{A}^{5}$ of the union of two affine hyperplanes $H_{0}=\left\{u_{0}=0\right\}$ and $H_{1}=\left\{u_{0}=1\right\}$ and the hypersurface $\widehat{C Z}_{\alpha}$ defined by the vanishing of the quadratic form

$$
Q_{\alpha, 2}=u_{1}^{2}+\alpha^{-2}\left(u_{2}^{2}+u_{3}^{2}+u_{4}^{2}\right) .
$$

Proof. We use the formula (3.2) in the special case of $r=0$ to calculate the term $a_{2}$ appearing in the heat kernel expansion (3.1). In this case we have

$$
a_{2}=\frac{1}{2^{5} \pi^{4}} \operatorname{Res}\left(\left(D^{2}\right)^{-1}\right)
$$

where $\left(D^{2}\right)^{-1}$ denotes the parametrix of $D^{2}$. In order to use the formula (3.3), since the dimension of the manifold is 4 , we need to calculate the term $\sigma_{-4}(x, \xi)$ that is homogeneous of order -4 in the expansion of the symbol of $\left(D^{2}\right)^{-1}$. By performing symbolic calculations we find that:

$$
\operatorname{tr}\left(\sigma_{-4}(x, \xi)\right)=
$$




$$
\begin{aligned}
& \frac{32 \cot ^{2}(\eta) \xi_{3}^{4} \csc ^{4}(\eta)}{a(t)^{6}\left(\xi_{1}^{2}+\frac{\xi_{2}^{2}}{a(t)^{2}}+\frac{\csc ^{2}(\eta) \xi_{3}^{2}}{a(t)^{2}}+\frac{\sec ^{2}(\eta) \xi_{4}^{2}}{a(t)^{2}}\right)_{4}}+\frac{32 \xi_{2}^{2} \xi_{3}^{2} \csc ^{4}(\eta)}{a(t)^{6}\left(\xi_{1}^{2}+\frac{\xi_{2}^{2}}{a(t)^{2}}+\frac{\csc ^{2}(\eta) \xi_{3}^{2}}{a(t)^{2}}+\frac{\sec ^{2}(\eta) \xi_{4}^{2}}{a(t)^{2}}\right)_{4}}+ \\
& \frac{32 \xi_{3}^{4} a^{\prime}(t)^{2} \csc ^{4}(\eta)}{a(t)^{6}\left(\xi_{1}^{2}+\frac{\xi_{2}^{2}}{a(t)^{2}}+\frac{\csc ^{2}(\eta) \xi_{3}^{2}}{a(t)^{2}}+\frac{\sec ^{2}(\eta) \xi_{4}^{2}}{a(t)^{2}}\right)^{4}}-\frac{8 \xi_{3}^{2} \csc ^{4}(\eta)}{a(t)^{4}\left(\xi_{1}^{2}+\frac{\xi_{2}^{2}}{a(t)^{2}}+\frac{\csc ^{2}(\eta) \xi_{3}^{2}}{a(t)^{2}}+\frac{\sec ^{2}(\eta) \xi_{4}^{2}}{a(t)^{2}}\right)^{3}}-
\end{aligned}
$$

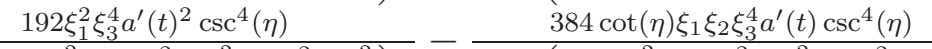

$$
\begin{aligned}
& \overline{a(t)^{6}\left(\xi_{1}^{2}+\frac{\xi_{2}^{2}}{a(t)^{2}}+\frac{\csc ^{2}(\eta) \xi_{3}^{2}}{a(t)^{2}}+\frac{\sec ^{2}(\eta) \xi_{4}^{2}}{a(t)^{2}}\right)_{5}}-\frac{\xi_{2}^{2}}{a(t)^{7}\left(\xi_{1}^{2}+\frac{\xi_{2}^{2}}{a(t)^{2}}+\frac{\csc ^{2}(\eta) \xi_{3}^{2}}{a(t)^{2}}+\frac{\sec ^{2}(\eta) \xi_{4}^{2}}{a(t)^{2}}\right)_{5}}-
\end{aligned}
$$

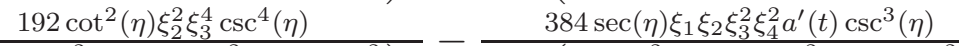

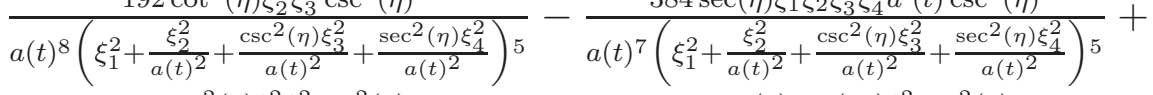

$$
\begin{aligned}
& \frac{64 \cot ^{2}(\eta) \xi_{2}^{2} \xi_{3}^{2} \csc ^{2}(\eta)}{a(t)^{6}\left(\xi_{1}^{2}+\frac{\xi_{2}^{2}}{a(t)^{2}}+\frac{\csc ^{2}(\eta) \xi_{3}^{2}}{a(t)^{2}}+\frac{\sec ^{2}(\eta) \xi_{4}^{2}}{a(t)^{2}}\right)^{4}}+\frac{16 \cot (\eta) \cot (2 \eta) \xi_{3}^{2} \csc ^{2}(\eta)}{a(t)^{4}\left(\xi_{1}^{2}+\frac{\xi_{2}^{2}}{a(t)^{2}}+\frac{\csc ^{2}(\eta) \xi_{3}^{2}}{a(t)^{2}}+\frac{\sec ^{2}(\eta) \xi_{4}^{2}}{a(t)^{2}}\right)^{3}}+ \\
& \frac{384 \sec ^{2}(\eta) \xi_{2}^{2} \xi_{3}^{2} \xi_{4}^{2} \csc ^{2}(\eta)}{a(t)^{8}\left(\xi_{1}^{2}+\frac{\xi_{2}^{2}}{a(t)^{2}}+\frac{\csc ^{2}(\eta) \xi_{3}^{2}}{a(t)^{2}}+\frac{\sec ^{2}(\eta) \xi_{4}^{2}}{a(t)^{2}}\right)^{5}}+\frac{64 \xi_{2}^{2} \xi_{3}^{2} a^{\prime}(t)^{2} \csc ^{2}(\eta)}{a(t)^{6}\left(\xi_{1}^{2}+\frac{\xi_{2}^{2}}{a(t)^{2}}+\frac{\csc ^{2}(\eta) \xi_{3}^{2}}{a(t)^{2}}+\frac{\sec ^{2}(\eta) \xi_{4}^{2}}{a(t)^{2}}\right)_{4}}+ \\
& \frac{4 \xi_{3}^{2} a^{\prime}(t)^{2} \csc ^{2}(\eta)}{a(t)^{4}\left(\xi_{1}^{2}+\frac{\xi_{2}^{2}}{a(t)^{2}}+\frac{\csc ^{2}(\eta) \xi_{3}^{2}}{a(t)^{2}}+\frac{\sec ^{2}(\eta) \xi_{4}^{2}}{a(t)^{2}}\right)^{3}}+\frac{64 \sec ^{2}(\eta) \xi_{3}^{2} \xi_{4}^{2} a^{\prime}(t)^{2} \csc ^{2}(\eta)}{a(t)^{6}\left(\xi_{1}^{2}+\frac{\xi_{2}^{2}}{a(t)^{2}}+\frac{\csc ^{2}(\eta) \xi_{3}^{2}}{a(t)^{2}}+\frac{\sec ^{2}(\eta) \xi_{4}^{2}}{a(t)^{2}}\right)_{4}}+ \\
& \frac{48 \cot (\eta) \xi_{1} \xi_{2} \xi_{3}^{2} a^{\prime}(t) \csc ^{2}(\eta)}{a(t)^{5}\left(\xi_{1}^{2}+\frac{\xi_{2}^{2}}{a(t)^{2}}+\frac{\csc ^{2}(\eta) \xi_{3}^{2}}{a(t)^{2}}+\frac{\sec ^{2}(\eta) \xi_{4}^{2}}{a(t)^{2}}\right)^{4}}+\frac{8 \xi_{3}^{2} a^{\prime \prime}(t) \csc ^{2}(\eta)}{a(t)^{3}\left(\xi_{1}^{2}+\frac{\xi_{2}^{2}}{a(t)^{2}}+\frac{\csc ^{2}(\eta) \xi_{3}^{2}}{a(t)^{2}}+\frac{\sec ^{2}(\eta) \xi_{4}^{2}}{a(t)^{2}}\right)^{3}}- \\
& \frac{\csc ^{2}(\eta)}{a(t)^{2}\left(\xi_{1}^{2}+\frac{\xi_{2}^{2}}{a(t)^{2}}+\frac{\csc ^{2}(\eta) \xi_{3}^{2}}{a(t)^{2}}+\frac{\sec ^{2}(\eta) \xi_{4}^{2}}{a(t)^{2}}\right)^{2}}-\frac{12 \cot ^{2}(\eta) \xi_{3}^{2} \csc ^{2}(\eta)}{a(t)^{4}\left(\xi_{1}^{2}+\frac{\xi_{2}^{2}}{a(t)^{2}}+\frac{\csc ^{2}(\eta) \xi_{3}^{2}}{a(t)^{2}}+\frac{\sec ^{2}(\eta) \xi_{4}^{2}}{a(t)^{2}}\right)^{3}}- \\
& \frac{32 \xi_{1}^{2} \xi_{3}^{2} a^{\prime \prime}(t) \csc ^{2}(\eta)}{a(t)^{3}\left(\xi_{1}^{2}+\frac{\xi_{2}^{2}}{a(t)^{2}}+\frac{\csc ^{2}(\eta) \xi_{3}^{2}}{a(t)^{2}}+\frac{\sec ^{2}(\eta) \xi_{4}^{2}}{a(t)^{2}}\right)^{4}}-\frac{48 \xi_{1}^{2} \xi_{3}^{2} a^{\prime}(t)^{2} \csc ^{2}(\eta)}{a(t)^{4}\left(\xi_{1}^{2}+\frac{\xi_{2}^{2}}{a(t)^{2}}+\frac{\csc ^{2}(\eta) \xi_{3}^{2}}{a(t)^{2}}+\frac{\sec ^{2}(\eta) \xi_{4}^{2}}{a(t)^{2}}\right)^{4}}- \\
& \frac{96 \cot (2 \eta) \xi_{1} \xi_{2} \xi_{3}^{2} a^{\prime}(t) \csc ^{2}(\eta)}{a(t)^{5}\left(\xi_{1}^{2}+\frac{\xi_{2}^{2}}{a(t)^{2}}+\frac{\csc ^{2}(\eta) \xi_{3}^{2}}{a(t)^{2}}+\frac{\sec ^{2}(\eta) \xi_{4}^{2}}{a(t)^{2}}\right)^{4}}-\frac{96 \cot (\eta) \cot (2 \eta) \xi_{2}^{2} \xi_{3}^{2} \csc ^{2}(\eta)}{a(t)^{6}\left(\xi_{1}^{2}+\frac{\xi_{2}^{2}}{a(t)^{2}}+\frac{\csc ^{2}(\eta) \xi_{3}^{2}}{a(t)^{2}}+\frac{\sec ^{2}(\eta) \xi_{4}^{2}}{a(t)^{2}}\right)_{4}}- \\
& \frac{64 \sec ^{2}(\eta) \xi_{3}^{2} \xi_{4}^{2} \csc ^{2}(\eta)}{a(t)^{6}\left(\xi_{1}^{2}+\frac{\xi_{2}^{2}}{a(t)^{2}}+\frac{\csc ^{2}(\eta) \xi_{3}^{2}}{a(t)^{2}}+\frac{\sec ^{2}(\eta) \xi_{4}^{2}}{a(t)^{2}}\right)^{4}}-\frac{384 \xi_{1}^{2} \xi_{2}^{2} \xi_{3}^{2} a^{\prime}(t)^{2} \csc ^{2}(\eta)}{a(t)^{6}\left(\xi_{1}^{2}+\frac{\xi_{2}^{2}}{a(t)^{2}}+\frac{\csc ^{2}(\eta) \xi_{3}^{2}}{a(t)^{2}}+\frac{\sec ^{2}(\eta) \xi_{4}^{2}}{a(t)^{2}}\right)_{5}}- \\
& \frac{384 \sec ^{2}(\eta) \xi_{1}^{2} \xi_{3}^{2} \xi_{4}^{2} a^{\prime}(t)^{2} \csc ^{2}(\eta)}{a(t)^{6}\left(\xi_{1}^{2}+\frac{\xi_{2}^{2}}{a(t)^{2}}+\frac{\csc ^{2}(\eta) \xi_{3}^{2}}{a(t)^{2}}+\frac{\sec ^{2}(\eta) \xi_{4}^{2}}{a(t)^{2}}\right)^{5}}-\frac{384 \cot (\eta) \xi_{1} \xi_{2}^{3} \xi_{3}^{2} a^{\prime}(t) \csc ^{2}(\eta)}{a(t)^{7}\left(\xi_{1}^{2}+\frac{\xi_{2}^{2}}{a(t)^{2}}+\frac{\csc ^{2}(\eta) \xi_{3}^{2}}{a(t)^{2}}+\frac{\sec ^{2}(\eta) \xi_{4}^{2}}{a(t)^{2}}\right)^{5}}+ \\
& \frac{384 \sec ^{3}(\eta) \xi_{1} \xi_{2} \xi_{3}^{2} \xi_{4}^{2} a^{\prime}(t) \csc (\eta)}{a(t)^{7}\left(\xi_{1}^{2}+\frac{\xi_{2}^{2}}{a(t)^{2}}+\frac{\csc ^{2}(\eta) \xi_{3}^{2}}{a(t)^{2}}+\frac{\sec ^{2}(\eta) \xi_{4}^{2}}{a(t)^{2}}\right)^{5}}+\frac{32 \csc ^{2}(2 \eta) \xi_{2}^{2}}{a(t)^{4}\left(\xi_{1}^{2}+\frac{\xi_{2}^{2}}{a(t)^{2}}+\frac{\csc ^{2}(\eta) \xi_{3}^{2}}{a(t)^{2}}+\frac{\sec ^{2}(\eta) \xi_{4}^{2}}{a(t)^{2}}\right)^{3}}+ \\
& \frac{32 \sec ^{4}(\eta) \xi_{2}^{2} \xi_{4}^{2}}{a(t)^{6}\left(\xi_{1}^{2}+\frac{\xi_{2}^{2}}{a(t)^{2}}+\frac{\csc ^{2}(\eta) \xi_{3}^{2}}{a(t)^{2}}+\frac{\sec ^{2}(\eta) \xi_{4}^{2}}{a(t)^{2}}\right) 4}+\frac{32 \sec ^{4}(\eta) \xi_{4}^{4} \tan ^{2}(\eta)}{a(t)^{6}\left(\xi_{1}^{2}+\frac{\xi_{2}^{2}}{a(t)^{2}}+\frac{\csc ^{2}(\eta) \xi_{3}^{2}}{a(t)^{2}}+\frac{\sec ^{2}(\eta) \xi_{4}^{2}}{a(t)^{2}}\right)_{4}}+ \\
& \frac{64 \sec ^{2}(\eta) \xi_{2}^{2} \xi_{4}^{2} \tan ^{2}(\eta)}{a(t)^{6}\left(\xi_{1}^{2}+\frac{\xi_{2}^{2}}{a(t)^{2}}+\frac{\csc ^{2}(\eta) \xi_{3}^{2}}{a(t)^{2}}+\frac{\sec ^{2}(\eta) \xi_{4}^{2}}{a(t)^{2}}\right)^{4}}+\frac{32 \xi_{2}^{4} a^{\prime}(t)^{2}}{a(t)^{6}\left(\xi_{1}^{2}+\frac{\xi_{2}^{2}}{a(t)^{2}}+\frac{\csc ^{2}(\eta) \xi_{3}^{2}}{a(t)^{2}}+\frac{\sec ^{2}(\eta) \xi_{4}^{2}}{a(t)^{2}}\right)^{4}}+ \\
& \frac{32 \sec ^{4}(\eta) \xi_{4}^{4} a^{\prime}(t)^{2}}{a(t)^{6}\left(\xi_{1}^{2}+\frac{\xi_{2}^{2}}{a(t)^{2}}+\frac{\csc ^{2}(\eta) \xi_{3}^{2}}{a(t)^{2}}+\frac{\sec ^{2}(\eta) \xi_{4}^{2}}{a(t)^{2}}\right)^{4}}+\frac{4 \xi_{2}^{2} a^{\prime}(t)^{2}}{a(t)^{4}\left(\xi_{1}^{2}+\frac{\xi_{2}^{2}}{a(t)^{2}}+\frac{\csc ^{2}(\eta) \xi_{3}^{2}}{a(t)^{2}}+\frac{\sec ^{2}(\eta) \xi_{4}^{2}}{a(t)^{2}}\right)^{3}}+ \\
& \frac{4 \sec ^{2}(\eta) \xi_{4}^{2} a^{\prime}(t)^{2}}{a(t)^{4}\left(\xi_{1}^{2}+\frac{\xi_{2}^{2}}{a(t)^{2}}+\frac{\csc ^{2}(\eta) \xi_{3}^{2}}{a(t)^{2}}+\frac{\sec ^{2}(\eta) \xi_{4}^{2}}{a(t)^{2}}\right)^{3}}+\frac{64 \sec ^{2}(\eta) \xi_{2}^{2} \xi_{4}^{2} a^{\prime}(t)^{2}}{a(t)^{6}\left(\xi_{1}^{2}+\frac{\xi_{2}^{2}}{a(t)^{2}}+\frac{\csc ^{2}(\eta) \xi_{3}^{2}}{a(t)^{2}}+\frac{\sec ^{2}(\eta) \xi_{4}^{2}}{a(t)^{2}}\right)^{4}}+ \\
& \frac{3 a^{\prime}(t)^{2}}{a(t)^{2}\left(\xi_{1}^{2}+\frac{\xi_{2}^{2}}{a(t)^{2}}+\frac{\csc ^{2}(\eta) \xi_{3}^{2}}{a(t)^{2}}+\frac{\sec ^{2}(\eta) \xi_{4}^{2}}{a(t)^{2}}\right)^{2}}+\frac{96 \cot (2 \eta) \sec ^{2}(\eta) \xi_{2}^{2} \xi_{4}^{2} \tan (\eta)}{a(t)^{6}\left(\xi_{1}^{2}+\frac{\xi_{2}^{2}}{a(t)^{2}}+\frac{\csc ^{2}(\eta) \xi_{3}^{2}}{a(t)^{2}}+\frac{\sec ^{2}(\eta) \xi_{4}^{2}}{a(t)^{2}}\right)_{4}}+ \\
& \frac{384 \sec ^{4}(\eta) \xi_{1} \xi_{2} \xi_{4}^{4} \tan (\eta) a^{\prime}(t)}{a(t)^{7}\left(\xi_{1}^{2}+\frac{\xi_{2}^{2}}{a(t)^{2}}+\frac{\csc ^{2}(\eta) \xi_{3}^{2}}{a(t)^{2}}+\frac{\sec ^{2}(\eta) \xi_{4}^{2}}{a(t)^{2}}\right)_{5}}+\frac{384 \sec ^{2}(\eta) \xi_{1} \xi_{2}^{3} \xi_{4}^{2} \tan (\eta) a^{\prime}(t)}{a(t)^{7}\left(\xi_{1}^{2}+\frac{\xi_{2}^{2}}{a(t)^{2}}+\frac{\csc ^{2}(\eta) \xi_{3}^{2}}{a(t)^{2}}+\frac{\sec ^{2}(\eta) \xi_{4}^{2}}{a(t)^{2}}\right)_{5}}+
\end{aligned}
$$




$$
\begin{aligned}
& \frac{8 \xi_{2}^{2} a^{\prime \prime}(t)}{a(t)^{3}\left(\xi_{1}^{2}+\frac{\xi_{2}^{2}}{a(t)^{2}}+\frac{\csc ^{2}(\eta) \xi_{3}^{2}}{a(t)^{2}}+\frac{\sec ^{2}(\eta) \xi_{4}^{2}}{a(t)^{2}}\right)^{3}}+\frac{8 \sec ^{2}(\eta) \xi_{4}^{2} a^{\prime \prime}(t)}{a(t)^{3}\left(\xi_{1}^{2}+\frac{\xi_{2}^{2}}{a(t)^{2}}+\frac{\csc ^{2}(\eta) \xi_{3}^{2}}{a(t)^{2}}+\frac{\sec ^{2}(\eta) \xi_{4}^{2}}{a(t)^{2}}\right)^{3}}+ \\
& \frac{6 a^{\prime \prime}(t)}{a(t)\left(\xi_{1}^{2}+\frac{\xi_{2}^{2}}{a(t)^{2}}+\frac{\csc ^{2}(\eta) \xi_{3}^{2}}{a(t)^{2}}+\frac{\sec ^{2}(\eta) \xi_{4}^{2}}{a(t)^{2}}\right)^{2}}-\frac{\sec ^{2}(\eta)}{a(t)^{2}\left(\xi_{1}^{2}+\frac{\xi_{2}^{2}}{a(t)^{2}}+\frac{\csc ^{2}(\eta) \xi_{3}^{2}}{a(t)^{2}}+\frac{\sec ^{2}(\eta) \xi_{4}^{2}}{a(t)^{2}}\right)^{2}}- \\
& \frac{4}{a(t)^{2}\left(\xi_{1}^{2}+\frac{\xi_{2}^{2}}{a(t)^{2}}+\frac{\csc ^{2}(\eta) \xi_{3}^{2}}{a(t)^{2}}+\frac{\sec ^{2}(\eta) \xi_{4}^{2}}{a(t)^{2}}\right)^{2}}-\frac{24 \xi_{1}^{2} a^{\prime \prime}(t)}{a(t)\left(\xi_{1}^{2}+\frac{\xi_{2}^{2}}{a(t)^{2}}+\frac{\csc ^{2}(\eta) \xi_{3}^{2}}{a(t)^{2}}+\frac{\sec ^{2}(\eta) \xi_{4}^{2}}{a(t)^{2}}\right)^{3}}- \\
& \frac{12 \xi_{1}^{2} a^{\prime}(t)^{2}}{a(t)^{2}\left(\xi_{1}^{2}+\frac{\xi_{2}^{2}}{a(t)^{2}}+\frac{\csc ^{2}(\eta) \xi_{3}^{2}}{a(t)^{2}}+\frac{\sec ^{2}(\eta) \xi_{4}^{2}}{a(t)^{2}}\right)^{3}}-\frac{16 \cot (2 \eta) \xi_{1} \xi_{2} a^{\prime}(t)}{a(t)^{3}\left(\xi_{1}^{2}+\frac{\xi_{2}^{2}}{a(t)^{2}}+\frac{\csc ^{2}(\eta) \xi_{3}^{2}}{a(t)^{2}}+\frac{\sec ^{2}(\eta) \xi_{4}^{2}}{a(t)^{2}}\right)^{3}}- \\
& \frac{16 \cot ^{2}(2 \eta) \xi_{2}^{2}}{a(t)^{4}\left(\xi_{1}^{2}+\frac{\xi_{2}^{2}}{a(t)^{2}}+\frac{\csc ^{2}(\eta) \xi_{3}^{2}}{a(t)^{2}}+\frac{\sec ^{2}(\eta) \xi_{4}^{2}}{a(t)^{2}}\right)^{3}}-\frac{8 \sec ^{4}(\eta) \xi_{4}^{2}}{a(t)^{4}\left(\xi_{1}^{2}+\frac{\xi_{2}^{2}}{a(t)^{2}}+\frac{\csc ^{2}(\eta) \xi_{3}^{2}}{a(t)^{2}}+\frac{\sec ^{2}(\eta) \xi_{4}^{2}}{a(t)^{2}}\right)^{3}}- \\
& \frac{12 \sec ^{2}(\eta) \xi_{4}^{2} \tan ^{2}(\eta)}{a(t)^{4}\left(\xi_{1}^{2}+\frac{\xi_{2}^{2}}{a(t)^{2}}+\frac{\csc ^{2}(\eta) \xi_{3}^{2}}{a(t)^{2}}+\frac{\sec ^{2}(\eta) \xi_{4}^{2}}{a(t)^{2}}\right)^{3}}-\frac{16 \cot (2 \eta) \sec ^{2}(\eta) \xi_{4}^{2} \tan (\eta)}{a(t)^{4}\left(\xi_{1}^{2}+\frac{\xi_{2}^{2}}{a(t)^{2}}+\frac{\csc ^{2}(\eta) \xi_{3}^{2}}{a(t)^{2}}+\frac{\sec ^{2}(\eta) \xi_{4}^{2}}{a(t)^{2}}\right)^{3}}- \\
& \frac{32 \xi_{1}^{2} \xi_{2}^{2} a^{\prime \prime}(t)}{a(t)^{3}\left(\xi_{1}^{2}+\frac{\xi_{2}^{2}}{a(t)^{2}}+\frac{\csc ^{2}(\eta) \xi_{3}^{2}}{a(t)^{2}}+\frac{\sec ^{2}(\eta) \xi_{4}^{2}}{a(t)^{2}}\right) 4}-\frac{32 \sec ^{2}(\eta) \xi_{1}^{2} \xi_{4}^{2} a^{\prime \prime}(t)}{a(t)^{3}\left(\xi_{1}^{2}+\frac{\xi_{2}^{2}}{a(t)^{2}}+\frac{\csc ^{2}(\eta) \xi_{3}^{2}}{a(t)^{2}}+\frac{\sec ^{2}(\eta) \xi_{4}^{2}}{a(t)^{2}}\right)_{4}}- \\
& \frac{48 \xi_{1}^{2} \xi_{2}^{2} a^{\prime}(t)^{2}}{a(t)^{4}\left(\xi_{1}^{2}+\frac{\xi_{2}^{2}}{a(t)^{2}}+\frac{\csc ^{2}(\eta) \xi_{3}^{2}}{a(t)^{2}}+\frac{\sec ^{2}(\eta) \xi_{4}^{2}}{a(t)^{2}}\right)^{4}}-\frac{48 \sec ^{2}(\eta) \xi_{1}^{2} \xi_{4}^{2} a^{\prime}(t)^{2}}{a(t)^{4}\left(\xi_{1}^{2}+\frac{\xi_{2}^{2}}{a(t)^{2}}+\frac{\csc ^{2}(\eta) \xi_{3}^{2}}{a(t)^{2}}+\frac{\sec ^{2}(\eta) \xi_{4}^{2}}{a(t)^{2}}\right)^{4}}- \\
& \frac{96 \cot (2 \eta) \xi_{1} \xi_{2}^{3} a^{\prime}(t)}{a(t)^{5}\left(\xi_{1}^{2}+\frac{\xi_{2}^{2}}{a(t)^{2}}+\frac{\csc ^{2}(\eta) \xi_{3}^{2}}{a(t)^{2}}+\frac{\sec ^{2}(\eta) \xi_{4}^{2}}{a(t)^{2}}\right)^{4}}-\frac{96 \cot (2 \eta) \sec ^{2}(\eta) \xi_{1} \xi_{2} \xi_{4}^{2} a^{\prime}(t)}{a(t)^{5}\left(\xi_{1}^{2}+\frac{\xi_{2}^{2}}{a(t)^{2}}+\frac{\csc ^{2}(\eta) \xi_{3}^{2}}{a(t)^{2}}+\frac{\sec ^{2}(\eta) \xi_{4}^{2}}{a(t)^{2}}\right)^{4}}- \\
& \frac{48 \sec ^{2}(\eta) \xi_{1} \xi_{2} \xi_{4}^{2} \tan (\eta) a^{\prime}(t)}{a(t)^{5}\left(\xi_{1}^{2}+\frac{\xi_{2}^{2}}{a(t)^{2}}+\frac{\csc ^{2}(\eta) \xi_{3}^{2}}{a(t)^{2}}+\frac{\sec ^{2}(\eta) \xi_{4}^{2}}{a(t)^{2}}\right)^{4}}-\frac{192 \xi_{1}^{2} \xi_{2}^{4} a^{\prime}(t)^{2}}{a(t)^{6}\left(\xi_{1}^{2}+\frac{\xi_{2}^{2}}{a(t)^{2}}+\frac{\csc ^{2}(\eta) \xi_{3}^{2}}{a(t)^{2}}+\frac{\sec ^{2}(\eta) \xi_{4}^{2}}{a(t)^{2}}\right)_{5}}- \\
& \frac{192 \sec ^{4}(\eta) \xi_{1}^{2} \xi_{4}^{4} a^{\prime}(t)^{2}}{a(t)^{6}\left(\xi_{1}^{2}+\frac{\xi_{2}^{2}}{a(t)^{2}}+\frac{\csc ^{2}(\eta) \xi_{3}^{2}}{a(t)^{2}}+\frac{\sec ^{2}(\eta) \xi_{4}^{2}}{a(t)^{2}}\right)^{5}}-\frac{384 \sec ^{2}(\eta) \xi_{1}^{2} \xi_{2}^{2} \xi_{4}^{2} a^{\prime}(t)^{2}}{a(t)^{6}\left(\xi_{1}^{2}+\frac{\xi_{2}^{2}}{a(t)^{2}}+\frac{\csc ^{2}(\eta) \xi_{3}^{2}}{a(t)^{2}}+\frac{\sec ^{2}(\eta) \xi_{4}^{2}}{a(t)^{2}}\right)^{5}}- \\
& \frac{192 \sec ^{4}(\eta) \xi_{2}^{2} \xi_{4}^{4} \tan ^{2}(\eta)}{a(t)^{8}\left(\xi_{1}^{2}+\frac{\xi_{2}^{2}}{a(t)^{2}}+\frac{\csc ^{2}(\eta) \xi_{3}^{2}}{a(t)^{2}}+\frac{\sec ^{2}(\eta) \xi_{4}^{2}}{a(t)^{2}}\right)_{5}^{5}} .
\end{aligned}
$$

One can see easily that each term in this expression that has an odd power of $\xi_{j}$ in its numerator will finally integrate to 0 when we perform the integration of the 1-density, using formulas (3.3) and (3.4). Therefore we can reduce to considering only the following expression in our calculation, which is obtained from the above expression for $\operatorname{tr}\left(\sigma_{-4}(x, \xi)\right)$ after eliminating any term that has an odd exponent in the numerator:

$$
\begin{gathered}
b_{-4}(x, \xi)=b_{-4}(t, \eta, \xi)= \\
\frac{32 \cot ^{2}(\eta) \xi_{3}^{4} \csc ^{4}(\eta)}{a(t)^{6}\left(\xi_{1}^{2}+\frac{\xi_{2}^{2}}{a(t)^{2}}+\frac{\csc ^{2}(\eta) \xi_{3}^{2}}{a(t)^{2}}+\frac{\sec ^{2}(\eta) \xi_{4}^{2}}{a(t)^{2}}\right) 4}+\frac{32 \xi_{2}^{2} \xi_{3}^{2} \csc ^{4}(\eta)}{a(t)^{6}\left(\xi_{1}^{2}+\frac{\xi_{2}^{2}}{a(t)^{2}}+\frac{\csc ^{2}(\eta) \xi_{3}^{2}}{a(t)^{2}}+\frac{\sec ^{2}(\eta) \xi_{4}^{2}}{a(t)^{2}}\right)_{4}}+ \\
\frac{32 \xi_{3}^{4} a^{\prime}(t)^{2} \csc ^{4}(\eta)}{a(t)^{6}\left(\xi_{1}^{2}+\frac{\xi_{2}^{2}}{a(t)^{2}}+\frac{\csc ^{2}(\eta) \xi_{3}^{2}}{a(t)^{2}}+\frac{\sec ^{2}(\eta) \xi_{4}^{2}}{a(t)^{2}}\right)_{4}}-\frac{8 \xi_{3}^{2} \csc ^{4}(\eta)}{a(t)^{4}\left(\xi_{1}^{2}+\frac{\xi_{2}^{2}}{a(t)^{2}}+\frac{\csc ^{2}(\eta) \xi_{3}^{2}}{a(t)^{2}}+\frac{\sec ^{2}(\eta) \xi_{4}^{2}}{a(t)^{2}}\right)_{3}}- \\
\frac{192 \xi_{1}^{2} \xi_{3}^{4} a^{\prime}(t)^{2} \csc ^{4}(\eta)}{a(t)^{6}\left(\xi_{1}^{2}+\frac{\xi_{2}^{2}}{a(t)^{2}}+\frac{\csc ^{2}(\eta) \xi_{3}^{2}}{a(t)^{2}}+\frac{\sec ^{2}(\eta) \xi_{4}^{2}}{a(t)^{2}}\right)_{5}}-\frac{192 \cot ^{2}(\eta) \xi_{2}^{2} \xi_{3}^{4} \csc ^{4}(\eta)}{a(t)^{8}\left(\xi_{1}^{2}+\frac{\xi_{2}^{2}}{a(t)^{2}}+\frac{\csc ^{2}(\eta) \xi_{3}^{2}}{a(t)^{2}}+\frac{\sec ^{2}(\eta) \xi_{4}^{2}}{a(t)^{2}}\right)_{5}}+ \\
\frac{64 \cot ^{2}(\eta) \xi_{2}^{2} \xi_{3}^{2} \csc ^{2}(\eta)}{a(t)^{6}\left(\xi_{1}^{2}+\frac{\xi_{2}^{2}}{a(t)^{2}}+\frac{\csc ^{2}(\eta) \xi_{3}^{2}}{a(t)^{2}}+\frac{\sec ^{2}(\eta) \xi_{4}^{2}}{a(t)^{2}}\right)_{4}}+\frac{16 \cot (\eta) \cot (2 \eta) \xi_{3}^{2} \csc ^{2}(\eta)}{a(t)^{4}\left(\xi_{1}^{2}+\frac{\xi_{2}^{2}}{a(t)^{2}}+\frac{\csc ^{2}(\eta) \xi_{3}^{2}}{a(t)^{2}}+\frac{\sec ^{2}(\eta) \xi_{4}^{2}}{a(t)^{2}}\right)_{3}}+
\end{gathered}
$$




$$
\begin{aligned}
& \frac{384 \sec ^{2}(\eta) \xi_{2}^{2} \xi_{3}^{2} \xi_{4}^{2} \csc ^{2}(\eta)}{a(t)^{8}\left(\xi_{1}^{2}+\frac{\xi_{2}^{2}}{a(t)^{2}}+\frac{\csc ^{2}(\eta) \xi_{3}^{2}}{a(t)^{2}}+\frac{\sec ^{2}(\eta) \xi_{4}^{2}}{a(t)^{2}}\right)_{5}}+\frac{64 \xi_{2}^{2} \xi_{3}^{2} a^{\prime}(t)^{2} \csc ^{2}(\eta)}{a(t)^{6}\left(\xi_{1}^{2}+\frac{\xi_{2}^{2}}{a(t)^{2}}+\frac{\csc ^{2}(\eta) \xi_{3}^{2}}{a(t)^{2}}+\frac{\sec ^{2}(\eta) \xi_{4}^{2}}{a(t)^{2}}\right)^{4}}+ \\
& \frac{4 \xi_{3}^{2} a^{\prime}(t)^{2} \csc ^{2}(\eta)}{a(t)^{4}\left(\xi_{1}^{2}+\frac{\xi_{2}^{2}}{a(t)^{2}}+\frac{\csc ^{2}(\eta) \xi_{3}^{2}}{a(t)^{2}}+\frac{\sec ^{2}(\eta) \xi_{4}^{2}}{a(t)^{2}}\right)^{3}}+\frac{64 \sec ^{2}(\eta) \xi_{3}^{2} \xi_{4}^{2} a^{\prime}(t)^{2} \csc ^{2}(\eta)}{a(t)^{6}\left(\xi_{1}^{2}+\frac{\xi_{2}^{2}}{a(t)^{2}}+\frac{\csc ^{2}(\eta) \xi_{3}^{2}}{a(t)^{2}}+\frac{\sec ^{2}(\eta) \xi_{4}^{2}}{a(t)^{2}}\right)_{4}}+ \\
& \frac{8 \xi_{3}^{2} a^{\prime \prime}(t) \csc ^{2}(\eta)}{a(t)^{3}\left(\xi_{1}^{2}+\frac{\xi_{2}^{2}}{a(t)^{2}}+\frac{\csc ^{2}(\eta) \xi_{3}^{2}}{a(t)^{2}}+\frac{\sec ^{2}(\eta) \xi_{4}^{2}}{a(t)^{2}}\right)^{3}}-\frac{\csc ^{2}(\eta)}{a(t)^{2}\left(\xi_{1}^{2}+\frac{\xi_{2}^{2}}{a(t)^{2}}+\frac{\csc ^{2}(\eta) \xi_{3}^{2}}{a(t)^{2}}+\frac{\sec ^{2}(\eta) \xi_{4}^{2}}{a(t)^{2}}\right)^{2}}- \\
& 12 \cot ^{2}(\eta) \xi_{3}^{2} \csc ^{2}(\eta)-\frac{32 \xi_{1}^{2} \xi_{3}^{2} a^{\prime \prime}(t) \csc ^{2}(\eta)}{-} \\
& \overline{a(t)^{4}\left(\xi_{1}^{2}+\frac{\xi_{2}^{2}}{a(t)^{2}}+\frac{\csc ^{2}(\eta) \xi_{3}^{2}}{a(t)^{2}}+\frac{\sec ^{2}(\eta) \xi_{4}^{2}}{a(t)^{2}}\right)^{3}}-\overline{a(t)^{3}\left(\xi_{1}^{2}+\frac{\xi_{2}^{2}}{a(t)^{2}}+\frac{\csc ^{2}(\eta) \xi_{3}^{2}}{a(t)^{2}}+\frac{\sec ^{2}(\eta) \xi_{4}^{2}}{a(t)^{2}}\right)_{4}} \\
& 48 \xi_{1}^{2} \xi_{3}^{2} a^{\prime}(t)^{2} \csc ^{2}(\eta) \quad 96 \cot (\eta) \cot (2 \eta) \xi_{2}^{2} \xi_{3}^{2} \csc ^{2}(\eta) \\
& \overline{a(t)^{4}\left(\xi_{1}^{2}+\frac{\xi_{2}^{2}}{a(t)^{2}}+\frac{\csc ^{2}(\eta) \xi_{3}^{2}}{a(t)^{2}}+\frac{\sec ^{2}(\eta) \xi_{4}^{2}}{a(t)^{2}}\right)^{4}}-\overline{a(t)^{6}\left(\xi_{1}^{2}+\frac{\xi_{2}^{2}}{a(t)^{2}}+\frac{\csc ^{2}(\eta) \xi_{3}^{2}}{a(t)^{2}}+\frac{\sec ^{2}(\eta) \xi_{4}^{2}}{a(t)^{2}}\right)^{4}}
\end{aligned}
$$

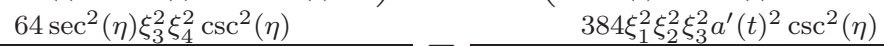

$$
\begin{aligned}
& \overline{a(t)^{6}\left(\xi_{1}^{2}+\frac{\xi_{2}^{2}}{a(t)^{2}}+\frac{\csc ^{2}(\eta) \xi_{3}^{2}}{a(t)^{2}}+\frac{\sec ^{2}(\eta) \xi_{4}^{2}}{a(t)^{2}}\right)^{4}}-\frac{\left.\xi_{1}^{2}+\frac{\xi_{2}^{2}}{a(t)^{2}}+\frac{\csc ^{2}(\eta) \xi_{3}^{2}}{a(t)^{2}}+\frac{\sec ^{2}(\eta) \xi_{4}^{2}}{a(t)^{2}}\right)_{5}}{-} \\
& \frac{384 \sec ^{2}(\eta) \xi_{1}^{2} \xi_{3}^{2} \xi_{4}^{2} a^{\prime}(t)^{2} \csc ^{2}(\eta)}{a(t)^{6}\left(\xi_{1}^{2}+\frac{\xi_{2}^{2}}{a(t)^{2}}+\frac{\csc ^{2}(\eta) \xi_{3}^{2}}{a(t)^{2}}+\frac{\sec ^{2}(\eta) \xi_{4}^{2}}{a(t)^{2}}\right)^{5}}+\frac{32 \csc ^{2}(2 \eta) \xi_{2}^{2}}{a(t)^{4}\left(\xi_{1}^{2}+\frac{\xi_{2}^{2}}{a(t)^{2}}+\frac{\csc ^{2}(\eta) \xi_{3}^{2}}{a(t)^{2}}+\frac{\sec ^{2}(\eta) \xi_{4}^{2}}{a(t)^{2}}\right)_{3}}+ \\
& \frac{32 \sec ^{4}(\eta) \xi_{2}^{2} \xi_{4}^{2}}{a(t)^{6}\left(\xi_{1}^{2}+\frac{\xi_{2}^{2}}{a(t)^{2}}+\frac{\csc ^{2}(\eta) \xi_{3}^{2}}{a(t)^{2}}+\frac{\sec ^{2}(\eta) \xi_{4}^{2}}{a(t)^{2}}\right)_{4}}+\frac{32 \sec ^{4}(\eta) \xi_{4}^{4} \tan ^{2}(\eta)}{a(t)^{6}\left(\xi_{1}^{2}+\frac{\xi_{2}^{2}}{a(t)^{2}}+\frac{\csc ^{2}(\eta) \xi_{3}^{2}}{a(t)^{2}}+\frac{\sec ^{2}(\eta) \xi_{4}^{2}}{a(t)^{2}}\right)_{4}}+ \\
& \frac{64 \sec ^{2}(\eta) \xi_{2}^{2} \xi_{4}^{2} \tan ^{2}(\eta)}{a(t)^{6}\left(\xi_{1}^{2}+\frac{\xi_{2}^{2}}{a(t)^{2}}+\frac{\csc ^{2}(\eta) \xi_{3}^{2}}{a(t)^{2}}+\frac{\sec ^{2}(\eta) \xi_{4}^{2}}{a(t)^{2}}\right)^{4}}+\frac{32 \xi_{2}^{4} a^{\prime}(t)^{2}}{a(t)^{6}\left(\xi_{1}^{2}+\frac{\xi_{2}^{2}}{a(t)^{2}}+\frac{\csc ^{2}(\eta) \xi_{3}^{2}}{a(t)^{2}}+\frac{\sec ^{2}(\eta) \xi_{4}^{2}}{a(t)^{2}}\right)_{4}}+ \\
& \frac{32 \sec ^{4}(\eta) \xi_{4}^{4} a^{\prime}(t)^{2}}{a(t)^{6}\left(\xi_{1}^{2}+\frac{\xi_{2}^{2}}{a(t)^{2}}+\frac{\csc ^{2}(\eta) \xi_{3}^{2}}{a(t)^{2}}+\frac{\sec ^{2}(\eta) \xi_{4}^{2}}{a(t)^{2}}\right)^{4}}+\frac{4 \xi_{2}^{2} a^{\prime}(t)^{2}}{a(t)^{4}\left(\xi_{1}^{2}+\frac{\xi_{2}^{2}}{a(t)^{2}}+\frac{\csc ^{2}(\eta) \xi_{3}^{2}}{a(t)^{2}}+\frac{\sec ^{2}(\eta) \xi_{4}^{2}}{a(t)^{2}}\right)_{3}}+ \\
& \frac{4 \sec ^{2}(\eta) \xi_{4}^{2} a^{\prime}(t)^{2}}{a(t)^{4}\left(\xi_{1}^{2}+\frac{\xi_{2}^{2}}{a(t)^{2}}+\frac{\csc ^{2}(\eta) \xi_{3}^{2}}{a(t)^{2}}+\frac{\sec ^{2}(\eta) \xi_{4}^{2}}{a(t)^{2}}\right)^{3}}+\frac{64 \sec ^{2}(\eta) \xi_{2}^{2} \xi_{4}^{2} a^{\prime}(t)^{2}}{a(t)^{6}\left(\xi_{1}^{2}+\frac{\xi_{2}^{2}}{a(t)^{2}}+\frac{\csc ^{2}(\eta) \xi_{3}^{2}}{a(t)^{2}}+\frac{\sec ^{2}(\eta) \xi_{4}^{2}}{a(t)^{2}}\right)^{4}}+ \\
& \frac{3 a^{\prime}(t)^{2}}{a(t)^{2}\left(\xi_{1}^{2}+\frac{\xi_{2}^{2}}{a(t)^{2}}+\frac{\csc ^{2}(\eta) \xi_{3}^{2}}{a(t)^{2}}+\frac{\sec ^{2}(\eta) \xi_{4}^{2}}{a(t)^{2}}\right)^{2}}+\frac{96 \cot (2 \eta) \sec ^{2}(\eta) \xi_{2}^{2} \xi_{4}^{2} \tan (\eta)}{a(t)^{6}\left(\xi_{1}^{2}+\frac{\xi_{2}^{2}}{a(t)^{2}}+\frac{\csc ^{2}(\eta) \xi_{3}^{2}}{a(t)^{2}}+\frac{\sec ^{2}(\eta) \xi_{4}^{2}}{a(t)^{2}}\right)^{4}}+ \\
& \frac{8 \xi_{2}^{2} a^{\prime \prime}(t)}{a(t)^{3}\left(\xi_{1}^{2}+\frac{\xi_{2}^{2}}{a(t)^{2}}+\frac{\csc ^{2}(\eta) \xi_{3}^{2}}{a(t)^{2}}+\frac{\sec ^{2}(\eta) \xi_{4}^{2}}{a(t)^{2}}\right)^{3}}+\frac{8 \sec ^{2}(\eta) \xi_{4}^{2} a^{\prime \prime}(t)}{a(t)^{3}\left(\xi_{1}^{2}+\frac{\xi_{2}^{2}}{a(t)^{2}}+\frac{\csc ^{2}(\eta) \xi_{3}^{2}}{a(t)^{2}}+\frac{\sec ^{2}(\eta) \xi_{4}^{2}}{a(t)^{2}}\right)^{3}}+ \\
& \frac{6 a^{\prime \prime}(t)}{a(t)\left(\xi_{1}^{2}+\frac{\xi_{2}^{2}}{a(t)^{2}}+\frac{\csc ^{2}(\eta) \xi_{3}^{2}}{a(t)^{2}}+\frac{\sec ^{2}(\eta) \xi_{4}^{2}}{a(t)^{2}}\right)^{2}}-\frac{\sec ^{2}(\eta)}{a(t)^{2}\left(\xi_{1}^{2}+\frac{\xi_{2}^{2}}{a(t)^{2}}+\frac{\csc ^{2}(\eta) \xi_{3}^{2}}{a(t)^{2}}+\frac{\sec ^{2}(\eta) \xi_{4}^{2}}{a(t)^{2}}\right)^{2}}- \\
& \frac{4}{a(t)^{2}\left(\xi_{1}^{2}+\frac{\xi_{2}^{2}}{a(t)^{2}}+\frac{\csc ^{2}(\eta) \xi_{3}^{2}}{a(t)^{2}}+\frac{\sec ^{2}(\eta) \xi_{4}^{2}}{a(t)^{2}}\right)^{2}}-\frac{24 \xi_{1}^{2} a^{\prime \prime}(t)}{a(t)\left(\xi_{1}^{2}+\frac{\xi_{2}^{2}}{a(t)^{2}}+\frac{\csc ^{2}(\eta) \xi_{3}^{2}}{a(t)^{2}}+\frac{\sec ^{2}(\eta) \xi_{4}^{2}}{a(t)^{2}}\right)^{3}}- \\
& \frac{12 \xi_{1}^{2} a^{\prime}(t)^{2}}{a(t)^{2}\left(\xi_{1}^{2}+\frac{\xi_{2}^{2}}{a(t)^{2}}+\frac{\csc ^{2}(\eta) \xi_{3}^{2}}{a(t)^{2}}+\frac{\sec ^{2}(\eta) \xi_{4}^{2}}{a(t)^{2}}\right)^{3}}-\frac{16 \cot ^{2}(2 \eta) \xi_{2}^{2}}{a(t)^{4}\left(\xi_{1}^{2}+\frac{\xi_{2}^{2}}{a(t)^{2}}+\frac{\csc ^{2}(\eta) \xi_{3}^{2}}{a(t)^{2}}+\frac{\sec ^{2}(\eta) \xi_{4}^{2}}{a(t)^{2}}\right)^{3}}- \\
& \frac{8 \sec ^{4}(\eta) \xi_{4}^{2}}{a(t)^{4}\left(\xi_{1}^{2}+\frac{\xi_{2}^{2}}{a(t)^{2}}+\frac{\csc ^{2}(\eta) \xi_{3}^{2}}{a(t)^{2}}+\frac{\sec ^{2}(\eta) \xi_{4}^{2}}{a(t)^{2}}\right)^{3}}-\frac{12 \sec ^{2}(\eta) \xi_{4}^{2} \tan ^{2}(\eta)}{a(t)^{4}\left(\xi_{1}^{2}+\frac{\xi_{2}^{2}}{a(t)^{2}}+\frac{\csc ^{2}(\eta) \xi_{3}^{2}}{a(t)^{2}}+\frac{\sec ^{2}(\eta) \xi_{4}^{2}}{a(t)^{2}}\right)^{3}}- \\
& \frac{16 \cot (2 \eta) \sec ^{2}(\eta) \xi_{4}^{2} \tan (\eta)}{a(t)^{4}\left(\xi_{1}^{2}+\frac{\xi_{2}^{2}}{a(t)^{2}}+\frac{\csc ^{2}(\eta) \xi_{3}^{2}}{a(t)^{2}}+\frac{\sec ^{2}(\eta) \xi_{4}^{2}}{a(t)^{2}}\right)^{3}}-\frac{32 \xi_{1}^{2} \xi_{2}^{2} a^{\prime \prime}(t)}{a(t)^{3}\left(\xi_{1}^{2}+\frac{\xi_{2}^{2}}{a(t)^{2}}+\frac{\csc ^{2}(\eta) \xi_{3}^{2}}{a(t)^{2}}+\frac{\sec ^{2}(\eta) \xi_{4}^{2}}{a(t)^{2}}\right)^{4}}- \\
& \frac{32 \sec ^{2}(\eta) \xi_{1}^{2} \xi_{4}^{2} a^{\prime \prime}(t)}{a(t)^{3}\left(\xi_{1}^{2}+\frac{\xi_{2}^{2}}{a(t)^{2}}+\frac{\csc ^{2}(\eta) \xi_{3}^{2}}{a(t)^{2}}+\frac{\sec ^{2}(\eta) \xi_{4}^{2}}{a(t)^{2}}\right)^{4}}-\frac{48 \xi_{1}^{2} \xi_{2}^{2} a^{\prime}(t)^{2}}{a(t)^{4}\left(\xi_{1}^{2}+\frac{\xi_{2}^{2}}{a(t)^{2}}+\frac{\csc ^{2}(\eta) \xi_{3}^{2}}{a(t)^{2}}+\frac{\sec ^{2}(\eta) \xi_{4}^{2}}{a(t)^{2}}\right)^{4}}- \\
& \frac{48 \sec ^{2}(\eta) \xi_{1}^{2} \xi_{4}^{2} a^{\prime}(t)^{2}}{a(t)^{4}\left(\xi_{1}^{2}+\frac{\xi_{2}^{2}}{a(t)^{2}}+\frac{\csc ^{2}(\eta) \xi_{3}^{2}}{a(t)^{2}}+\frac{\sec ^{2}(\eta) \xi_{4}^{2}}{a(t)^{2}}\right)^{4}}-\frac{192 \xi_{1}^{2} \xi_{2}^{4} a^{\prime}(t)^{2}}{a(t)^{6}\left(\xi_{1}^{2}+\frac{\xi_{2}^{2}}{a(t)^{2}}+\frac{\csc ^{2}(\eta) \xi_{3}^{2}}{a(t)^{2}}+\frac{\sec ^{2}(\eta) \xi_{4}^{2}}{a(t)^{2}}\right)^{5}}-
\end{aligned}
$$




$$
\begin{gathered}
\frac{192 \sec ^{4}(\eta) \xi_{1}^{2} \xi_{4}^{4} a^{\prime}(t)^{2}}{a(t)^{6}\left(\xi_{1}^{2}+\frac{\xi_{2}^{2}}{a(t)^{2}}+\frac{\csc ^{2}(\eta) \xi_{3}^{2}}{a(t)^{2}}+\frac{\sec ^{2}(\eta) \xi_{4}^{2}}{a(t)^{2}}\right)_{5}}-\frac{384 \sec ^{2}(\eta) \xi_{1}^{2} \xi_{2}^{2} \xi_{4}^{2} a^{\prime}(t)^{2}}{a(t)^{6}\left(\xi_{1}^{2}+\frac{\xi_{2}^{2}}{a(t)^{2}}+\frac{\csc ^{2}(\eta) \xi_{3}^{2}}{a(t)^{2}}+\frac{\sec ^{2}(\eta) \xi_{4}^{2}}{a(t)^{2}}\right)^{5}}- \\
\frac{192 \sec ^{4}(\eta) \xi_{2}^{2} \xi_{4}^{4} \tan ^{2}(\eta)}{a(t)^{8}\left(\xi_{1}^{2}+\frac{\xi_{2}^{2}}{a(t)^{2}}+\frac{\csc ^{2}(\eta) \xi_{3}^{2}}{a(t)^{2}}+\frac{\sec ^{2}(\eta) \xi_{4}^{2}}{a(t)^{2}}\right)_{5}} .
\end{gathered}
$$

Note that $b_{-4}$ as well as $\sigma_{-4}$ has no dependence on $\phi_{1}$ and $\phi_{2}$.

To express the expression in (4.2) as a rational function, we introduce the change of coordinates

$$
\begin{array}{ccc}
u_{0}=\sin ^{2}(\eta), & u_{1}=\xi_{1}, & u_{2}=\xi_{2}, \\
u_{3}=\csc (\eta) \xi_{3}, & u_{4}=\sec (\eta) \xi_{4}, &
\end{array}
$$

and we write $\alpha$ for the term $a(t)$, considering it as an affine variable, momentarily forgetting the time dependence, since we are omitting the time integration. The trigonometric expressions appearing in $b_{4}$ transform to the following expressions in the new coordinates:

$$
\begin{gathered}
\xi_{1}^{2}+\frac{\xi_{2}^{2}}{a(t)^{2}}+\frac{\xi_{3}^{2} \csc ^{2}(\eta)}{a(t)^{2}}+\frac{\xi_{4}^{2} \sec ^{2}(\eta)}{a(t)^{2}}=u_{1}^{2}+\frac{1}{a(t)^{2}}\left(u_{2}^{2}+u_{3}^{2}+u_{4}^{2}\right), \\
\cot ^{2}(\eta)=\frac{1-u_{0}}{u_{0}} \\
\csc ^{2}(\eta)=\frac{1}{u_{0}} \\
\sec ^{2}(\eta)=\frac{1}{1-u_{0}}, \\
\cot (\eta) \cot (2 \eta)=\frac{\cot ^{2}(\eta)}{2}-\frac{1}{2}, \\
\csc (2 \eta)=\frac{1}{4} \csc ^{2}(\eta) \sec ^{2}(\eta), \\
\tan ^{2}(\eta)=\sec ^{2}(\eta)-1, \\
\tan ^{2}(\eta) \cot (2 \eta)=\frac{1}{2}-\frac{\tan ^{2}(\eta)}{2}, \\
\tan ^{2}(\eta) \\
8 \quad \frac{\cot ^{2}(\eta)}{8}+\frac{1}{8} \csc ^{2}(\eta) \sec ^{2}(\eta)-\frac{3}{4}
\end{gathered}
$$

Moreover, the exponents of the variables $\xi_{j}$ in the expression (4.2) for $b_{-4}$ are even positive integers.

Thus, it is clear that with the change of variables (4.3) the expression above becomes a $\mathbb{Q}$-linear combination of rational functions with numerators that are rational polynomials in the variables $\left(u_{0}, u_{1}, u_{2}, u_{3}, u_{4}, \alpha\right)$ and with denominators that are all of the form $\alpha^{2 r} u_{0}^{k}\left(1-u_{0}\right)^{m}\left(u_{1}^{2}+\alpha^{-2}\left(u_{2}^{2}+u_{3}^{2}+u_{4}^{2}\right)\right)^{\ell}$, with $r, k, m, \ell \in \mathbb{Z}_{\geq 0}$.

We also have

$$
\sigma_{\xi, 3}=\sum_{j=1}^{4}(-1)^{j-1} \xi_{j} d \xi_{1} \wedge \cdots \wedge \widehat{d} \xi_{j} \wedge \cdots \wedge d \xi_{4}
$$


With the change of variables (4.3) we obtain

$$
\begin{aligned}
d \eta & =\frac{1}{2 \sin (\eta) \cos (\eta)} d u_{0}=\frac{1}{2} \csc (\eta) \sec (\eta) d u_{0} \\
d \xi_{1} & =d u_{1}, \\
d \xi_{2} & =d u_{2}, \\
d \xi_{3} & =\cos (\eta) u_{3} d \eta+\sin (\eta) d u_{3}, \\
d \xi_{4} & =-\sin (\eta) u_{4} d \eta+\cos (\eta) d u_{4},
\end{aligned}
$$

which yields

$$
\begin{aligned}
\tilde{\sigma}_{3}:= & d \eta \wedge \sigma_{\xi, 3} \\
= & \sum_{j=1}^{4}(-1)^{j-1} \xi_{j} d \eta \wedge d \xi_{1} \wedge \cdots \wedge \widehat{d} \xi_{j} \wedge \cdots \wedge d \xi_{4} \\
= & \sin (\eta) \cos (\eta)\left(u_{1} d \eta d u_{2} d u_{3} d u_{4}-u_{2} d \eta d u_{1} d u_{3} d u_{4}\right. \\
& \left.+u_{3} d \eta d u_{1} d u_{2} d u_{4}-u_{4} d \eta d u_{1} d u_{2} d u_{3}\right) \\
= & \frac{1}{2}\left(u_{1} d u_{0} d u_{2} d u_{3} d u_{4}-u_{2} d u_{0} d u_{1} d u_{3} d u_{4}+u_{3} d u_{0} d u_{1} d u_{2} d u_{4}\right. \\
&
\end{aligned}
$$

Thus we obtain an algebraic differential form $\Omega$, defined over $\mathbb{Q}$, whose singular locus is defined by the vanishing of the quadratic form

$$
u_{1}^{2}+\alpha^{-2}\left(u_{2}^{2}+u_{3}^{2}+u_{4}^{2}\right),
$$

where we assume $\alpha \in \mathbb{G}_{m}=\mathbb{A}^{1} \backslash\{0\}$. This quadratic form defines, for each fixed value of $\alpha$, a quadric surface $Z_{\alpha}$ in $\mathbb{P}^{3}$. We write $\hat{Z}_{\alpha}$ for the corresponding affine hypersurface in $\mathbb{A}^{4}$, the affine cone over $Z_{\alpha}$. We also denote by $C Z_{\alpha}$ the projective cone of $Z_{\alpha}$ in $\mathbb{P}^{4}$ and by $\widehat{C Z}_{\alpha}$ its affine cone in $\mathbb{A}^{5}$. The latter is a product of the hypersurface $\hat{Z}_{\alpha}$ in $\mathbb{A}^{4}$ with the line $\mathbb{A}^{1}$ of the $u_{0}$-coordinate.

The restriction $\Omega^{\alpha}$ of the form $\Omega$ to a fixed (rational) value of $\alpha$ determines a twoparameter family of rational differential forms, by viewing the two variables $\left(\alpha_{1}, \alpha_{2}\right)$ that appear in the numerators as parameters,

$$
P\left(u_{0}, u_{1}, u_{2}, u_{3}, u_{4}, \alpha, \alpha_{1}, \alpha_{2}\right)=P_{\left(\alpha_{1}, \alpha_{2}\right)}\left(u_{0}, u_{1}, u_{2}, u_{3}, u_{4}, \alpha\right),
$$

with $P_{\left(\alpha_{1}, \alpha_{2}\right)} \in \mathbb{Q}\left[u_{0}, u_{1}, u_{2}, u_{3}, u_{4}, \alpha\right]$ for all $\left(\alpha_{1}, \alpha_{2}\right) \in \mathbb{Q}^{2}$. For any choice of the parameters $\left(\alpha_{1}, \alpha_{2}\right) \in \mathbb{Q}^{2}$, the resulting form $\Omega_{\left(\alpha_{1}, \alpha_{2}\right)}^{\alpha}$ is an algebraic differential form on the same variety $\mathbb{A}^{5} \backslash\left(\widehat{C Z}_{\alpha} \cup H_{0} \cup H_{1}\right)$.

A $\mathbb{Q}$-semialgebraic set is a subset $S$ of some $\mathbb{R}^{n}$ that is of the form

$$
S=\left\{\left(x_{1}, \ldots, x_{n}\right) \in \mathbb{R}^{n}: P\left(x_{1}, \ldots, x_{n}\right) \geq 0\right\},
$$


for some polynomial $P \in \mathbb{Q}\left[x_{1}, \ldots, x_{n}\right]$, or obtained from such sets by taking a finite number of complements, intersections, and unions.

Theorem 4.2. When computed without performing the time integration, the $a_{2}$ term in the heat kernel expansion is a period integral

$$
C \cdot \int_{A} \Omega_{\left(\alpha_{1}, \alpha_{2}\right)}^{\alpha}
$$

with the algebraic differential form of Proposition 4.1, and with domain of integration the $\mathbb{Q}$-semialgebraic set

$$
A_{4}=\left\{\left(u_{0}, u_{1}, u_{2}, u_{3}, u_{4}\right) \in \mathbb{A}^{5}(\mathbb{R}): \begin{array}{l}
u_{1}^{2}+u_{2}^{2}+u_{0} u_{3}^{2}+\left(1-u_{0}\right) u_{4}^{2}=1, \\
0<u_{i}<1, \text { for } i=0,1,2
\end{array}\right\} .
$$

The coefficient $C$ is in $\mathbb{Q}\left[(2 \pi i)^{-1}\right]$. This integral is a period of the mixed motive

$$
\mathfrak{m}\left(\mathbb{A}^{5} \backslash\left(\widehat{C Z}_{\alpha} \cup H_{0} \cup H_{1}\right), \Sigma\right),
$$

where $\widehat{C Z}_{\alpha}$ is the hypersurface in $\mathbb{A}^{5}$ defined by the vanishing of the quadric $Q_{\alpha, 2}$ of (4.1), and $\Sigma=\cup_{i, a} H_{i, a}$ is the divisor given by the union of the hyperplanes $H_{i, a}=$ $\left\{u_{i}=a\right\}$, with $i \in\{0,1,2\}$ and $a \in\{0,1\}$.

Proof. With the notation (4.4) as above, and leaving out an integration with respect to $t$, we have

$$
\begin{aligned}
a_{2} & =\frac{1}{2^{5} \pi^{4}} \int_{0}^{\pi / 2} d \eta \int_{0}^{2 \pi} d \phi_{1} \int_{0}^{2 \pi} d \phi_{2} \int_{\xi_{1}^{2}+\xi_{2}^{2}+\xi_{3}^{2}+\xi_{4}^{2}=1} d^{3} \xi \cdot b_{-4}(t, \eta, \xi) \cdot \sigma_{\xi, 3} \\
& =\frac{1}{2^{3} \pi^{2}} \int_{0}^{\pi / 2} d \eta \int_{\xi_{1}^{2}+\xi_{2}^{2}+\xi_{3}^{2}+\xi_{4}^{2}=1} d^{3} \xi \cdot b_{-4}(t, \eta, \xi) \cdot \sigma_{\xi, 3} \\
& =\frac{1}{2^{3} \pi^{2}} \int_{\left(0, \frac{\pi}{2}\right) \times \mathbb{S}^{3}} b_{-4}(t, \eta, \xi) \sum_{j=1}^{4}(-1)^{j-1} \xi_{j} d \eta \wedge d \xi_{1} \wedge \cdots \wedge \widehat{d \xi}_{j} \wedge \cdots \wedge d \xi_{4} .
\end{aligned}
$$

We use the change of variables (4.3) as in Proposition 4.1 to rewrite both the form and the domain of integration and we obtain, up to a coefficient in $\mathbb{Q}\left[(2 \pi i)^{-1}\right]$, an integral of the form

$$
\int_{A} \Omega_{\left(\alpha_{1}, \alpha_{2}\right)}^{\alpha}
$$

with the form $\Omega_{\left(\alpha_{1}, \alpha_{2}\right)}^{\alpha}$ as obtained in Proposition 4.1. The same change of variables (4.3) transforms the domain of integration given by the set $\left(0, \frac{\pi}{2}\right) \times \mathbb{S}^{3}$ appearing in the integral (4.7), into the set

(4.8) $A_{4}:=\left\{\left(u_{0}, u_{1}, u_{2}, u_{3}, u_{4}\right) \in(0,1)^{3} \times \mathbb{R}^{2}: \quad u_{1}^{2}+u_{2}^{2}+u_{0} u_{3}^{2}+\left(1-u_{0}\right) u_{4}^{2}=1\right\}$,

which is a $\mathbb{Q}$-semialgebraic set. The form $\Omega^{\alpha}$ is defined on the complement in $\mathbb{A}^{5}$ of the union of the hyperplanes $H_{0}$ and $H_{1}$ and the hypersurface $\widehat{C Z}_{\alpha}$ given by the vanishing of the quadric $Q_{\alpha}$ of (4.1). Thus, it is an algebraic differential form on the algebraic variety $\mathbb{A}^{5} \backslash\left(\widehat{C Z}_{\alpha} \cup H_{0} \cup H_{1}\right)$. The domain of integration is not a closed 
cycle: it has a boundary $\partial A$ which is contained in the union of the hyperplanes $H_{i, a}=\left\{u_{i}=a\right\}$, with $i \in\{0,1,2\}$ and $a \in\{0,1\}$. Thus, the period corresponds to the relative motive $\mathfrak{m}\left(\mathbb{A}^{5} \backslash\left(\widehat{C Z}_{\alpha} \cup H_{0} \cup H_{1}\right), \Sigma\right)$, where the divisor is the union of these hyperplanes, $\Sigma=\cup_{i, a} H_{i, a}$.

Remark 4.3. The singular locus $\widehat{C Z}_{\alpha} \cup H_{0} \cup H_{1}$ of the algebraic differential form and the divisor $\Sigma$ containing the boundary of the domain of integration $A_{4}$ have nonempty intersection along $H_{0} \cup H_{1}$. However, unlike the case of quantum field theory where the intersection of the boundary of the domain of integration with the graph hypersurface is the source of infrared divergences, here we know a priori that the integral (4.5) is convergent, and so are all the other analogous integrals for the higher order $a_{2 n}$ terms, as one can see by computing them in the original spherical coordinates. Thus, we do not have a renormalization problem for these integrals.

\section{THE $a_{4}$ TERM AND QUADRIC HYPERSURFACES IN $\mathbb{P}^{5}$}

In order to compute the term $a_{4}$ appearing in the asymptotic expansion (3.1), we use formula (3.2) in the special case when $r$ is set equal to 2. That is, we need to consider the operator

$$
\Delta_{4}=D^{2} \otimes 1+1 \otimes \Delta_{\mathbb{T}^{2}}
$$

in which $\Delta_{\mathbb{T}^{2}}$ is the flat Laplacian on the 2-dimensional torus $\mathbb{T}^{2}=(\mathbb{R} / \mathbb{Z})^{2}$. The formula (3.2) allows us to write

$$
a_{4}=\frac{1}{2^{5} \pi^{5}} \operatorname{Res}\left(\Delta_{4}^{-1}\right) .
$$

Since the operator $\Delta_{4}$ and its parametrix $\Delta_{4}^{-1}$ act on the smooth sections of a vector bundle on a 6 -dimensional manifold, in order to compute $\operatorname{Res}\left(\Delta_{4}^{-1}\right)$, we first need to calculate $\operatorname{tr}\left(\sigma_{-6}\left(\Delta_{4}^{-1}\right)\right)$, where $\sigma_{-6}\left(\Delta_{4}^{-1}\right)$ is the term of order -6 in the expansion of the symbol of $\Delta_{4}^{-1}$.

This can be done by preforming symbolic calculations as explained in [8], which works for a general positive even integer $r$ and the operator

$$
\Delta_{r+2}=D^{2} \otimes 1+1 \otimes \Delta_{\mathbb{T}^{r}},
$$

in which $\Delta_{\mathbb{T}^{r}}$ is the flat Laplacian on the $r$-dimensional torus $\mathbb{T}^{r}=(\mathbb{R} / \mathbb{Z})^{r}$. In order to calculate the homogeneous terms in the expansion of the symbol of $\Delta_{r+2}^{-1}$, one can start by writing

$$
\sigma_{-2}\left(\Delta_{r+2}^{-1}\right)=\left(p_{2}\left(x, \xi_{1}, \xi_{2}, \xi_{3}, \xi_{4}\right)+\left(\xi_{5}^{2}+\cdots+\xi_{4+r}^{2}\right) I_{4 \times 4}\right)^{-1} .
$$

Then, the following formula, for $n>0$, can be used to calculate the next terms recursively:

$$
\sigma_{-2-n}\left(\Delta_{r+2}^{-1}\right)=
$$




$$
-\left(\sum_{\substack{0 \leq j<n, 0 \leq k \leq 2 \\ \alpha \in \mathbb{Z}_{\geq}^{4} \\-2-j-|\alpha|+k=-n}} \frac{(-i)^{|\alpha|}}{\alpha !}\left(\partial_{\xi}^{\alpha} \sigma_{-2-j}\left(\Delta_{r+2}^{-1}\right)\right)\left(\partial_{x}^{\alpha} p_{k}\right)\right) \sigma_{-2}\left(\Delta_{r+2}^{-1}\right) .
$$

Theorem 5.1. The form $b_{-6}=b_{-6}\left(t, \eta, \xi_{1}, \ldots, \xi_{6}\right)$ computing the $a_{4}$ term of the heat kernel expansion of $D^{2}$ is a rational differential form

$$
\Omega=f \widetilde{\sigma}_{5}
$$

in affine coordinates $\left(u_{0}, u_{1}, u_{2}, u_{3}, u_{4}, u_{5}, u_{6}\right) \in \mathbb{A}^{7}, \alpha \in \mathbb{G}_{m}$, and $\left(\alpha_{1}, \alpha_{2}, \alpha_{3}, \alpha_{4}\right) \in$ $\mathbb{A}^{4}$, where the functions

$$
f\left(u_{0}, u_{1}, u_{2}, u_{3}, u_{4}, \alpha, \alpha_{1}, \alpha_{2}, \alpha_{3}, \alpha_{4}\right)=f_{\left(\alpha_{1}, \alpha_{2}, \alpha_{3}, \alpha_{4}\right)}\left(u_{0}, u_{1}, u_{2}, u_{3}, u_{4}, u_{5}, u_{6}, \alpha\right)
$$

are $\mathbb{Q}$-linear combinations of rational functions of the form

$$
\frac{P\left(u_{0}, u_{1}, u_{2}, u_{3}, u_{4}, u_{5}, u_{6}, \alpha, \alpha_{1}, \alpha_{2}, \alpha_{3}, \alpha_{4}\right)}{\alpha^{2 r} u_{0}^{k}\left(1-u_{0}\right)^{m}\left(u_{1}^{2}+\alpha^{-2}\left(u_{2}^{2}+u_{3}^{2}+u_{4}^{2}\right)+u_{5}^{2}+u_{6}^{2}\right)^{\ell}}
$$

where

$$
P\left(u_{0}, u_{1}, u_{2}, u_{3}, u_{4}, u_{5}, u_{6}, \alpha, \alpha_{1}, \alpha_{2}, \alpha_{3}, \alpha_{4}\right)=P_{\left(\alpha_{1}, \alpha_{2}, \alpha_{3}, \alpha_{4}\right)}\left(u_{0}, u_{1}, u_{2}, u_{3}, u_{4}, u_{5}, u_{6}, \alpha\right)
$$

are polynomials in $\mathbb{Q}\left[u_{0}, u_{1}, u_{2}, u_{3}, u_{4}, u_{5}, u_{6} \alpha, \alpha_{1}, \alpha_{2}, \alpha_{3}, \alpha_{4}\right]$ and where $r, k, m$ and $\ell$ are non-negative integers, and with $\widetilde{\sigma}_{5}=\widetilde{\sigma}_{5}\left(u_{0}, u_{1}, u_{2}, u_{3}, u_{4}, u_{5}, u_{6}\right)$ the form

$$
\begin{aligned}
\tilde{\sigma}_{5}= & \frac{1}{2}\left(u_{1} d u_{0} d u_{2} d u_{3} d u_{4} d u_{5} d u_{6}-u_{2} d u_{0} d u_{1} d u_{3} d u_{4} d u_{5} d u_{6}\right. \\
& +u_{3} d u_{0} d u_{1} d u_{2} d u_{4} d u_{5} d u_{6}-u_{4} d u_{0} d u_{1} d u_{2} d u_{3} d u_{5} d u_{6} \\
& \left.+u_{5} d u_{0} d u_{1} d u_{2} d u_{3} d u_{4} d u_{6}-u_{6} d u_{0} d u_{1} d u_{2} d u_{3} d u_{4} d u_{5}\right) .
\end{aligned}
$$

The forms $\Omega^{\alpha}=\Omega_{\left(\alpha_{1}, \alpha_{2}, \alpha_{3}, \alpha_{4}\right)}^{\alpha}$ obtained by restricting the above to a fixed value of $\alpha \in \mathbb{A}^{1} \backslash\{0\}$ are a four-parameter family of algebraic differential forms on the algebraic variety over $\mathbb{Q}$ given by the complement in $\mathbb{A}^{7}$ of the union of the affine hyperplanes $H_{0}=\left\{u_{0}=0\right\}$ and $H_{1}=\left\{u_{0}=1\right\}$ and the hypersurface $\widehat{C Z}_{\alpha}$ defined by the vanishing of the quadratic form

$$
Q_{\alpha, 4}=u_{1}^{2}+\alpha^{-2}\left(u_{2}^{2}+u_{3}^{2}+u_{4}^{2}\right)+u_{5}^{2}+u_{6}^{2} .
$$

Proof. As we explained earlier, for the calculation of the term $a_{4}$, we need to set $r=2$. In this case, after performing the algebraic calculations we find a lengthy expression for $\operatorname{tr}\left(\sigma_{-6}\left(\Delta_{4}^{-1}\right)\right)$. Like the case of $a_{2}$, the expression for $\operatorname{tr}\left(\sigma_{-6}\left(\Delta_{4}^{-1}\right)\right)$ has terms that have odd powers of $\xi_{j}$ in their numerators. Since these terms, following formulas (3.3) and (3.4), will vanish under the necessary integrations for calculating $a_{4}$, we eliminate them from $\operatorname{tr}\left(\sigma_{-6}\left(\Delta_{4}^{-1}\right)\right)$ and denote the reduced expression by $b_{-6}$. Using the notation

$$
\alpha=a(t)
$$




$$
Q_{\alpha, 4}=\xi_{1}^{2}+\frac{\xi_{2}^{2}}{\alpha^{2}}+\frac{\xi_{3}^{2} \csc ^{2}(\eta)}{\alpha^{2}}+\frac{\xi_{4}^{2} \sec ^{2}(\eta)}{\alpha^{2}}+\xi_{5}^{2}+\xi_{6}^{2}
$$

we find that

$$
b_{-6}=b_{-6}\left(t, \eta, \xi_{1}, \ldots, \xi_{6}\right)=
$$

$$
\begin{aligned}
& \frac{2560 \xi_{3}^{8} a^{\prime}(t)^{4} \csc ^{8}(\eta)}{\alpha^{12} Q_{\alpha, 4}^{7}}-\frac{53760 \xi_{1}^{2} \xi_{3}^{8} a^{\prime}(t)^{4} \csc ^{8}(\eta)}{\alpha^{12} Q_{\alpha, 4}^{8}}+\frac{107520 \xi_{1}^{4} \xi_{3}^{8} a^{\prime}(t)^{4} \csc ^{8}(\eta)}{\alpha^{12} Q_{\alpha, 4}^{9}}-\frac{640 \xi_{3}^{6} a^{\prime}(t)^{2} \csc ^{8}(\eta)}{\alpha^{10} Q_{\alpha, 4}^{6}}+ \\
& \frac{5120 \cot ^{2}(\eta) \xi_{3}^{8} a^{\prime}(t)^{2} \csc ^{8}(\eta)}{\alpha^{12} Q_{\alpha, 4}^{7}}+\frac{5760 \xi_{1}^{2} \xi_{3}^{6} a^{\prime}(t)^{2} \csc ^{8}(\eta)}{\alpha^{10} Q_{\alpha, 4}^{7}}+\frac{5120 \xi_{2}^{2} \xi_{3}^{6} a^{\prime}(t)^{2} \csc ^{8}(\eta)}{\alpha^{12} Q_{\alpha, 4}^{7}}+\frac{10240 \xi_{3}^{6} \xi_{4}^{2} a^{\prime}(t)^{2} \csc ^{8}(\eta)}{\alpha^{12} Q_{\alpha, 4}^{7}}- \\
& \frac{53760 \cot ^{2}(\eta) \xi_{1}^{2} \xi_{3}^{8} a^{\prime}(t)^{2} \csc ^{8}(\eta)}{\alpha^{12} Q_{\alpha, 4}^{8}}-\frac{53760 \cot ^{2}(\eta) \xi_{2}^{2} \xi_{3}^{8} a^{\prime}(t)^{2} \csc ^{8}(\eta)}{\alpha^{14} Q_{\alpha, 4}^{8}}-\frac{53760 \xi_{1}^{2} \xi_{2}^{2} \xi_{3}^{6} a^{\prime}(t)^{2} \csc ^{8}(\eta)}{\alpha^{12} Q_{\alpha, 4}^{8}}- \\
& \frac{107520 \xi_{1}^{2} \xi_{3}^{6} \xi_{4}^{2} a^{\prime}(t)^{2} \csc ^{8}(\eta)}{\alpha^{12} Q_{\alpha, 4}^{8}}-\frac{107520 \xi_{2}^{2} \xi_{3}^{6} \xi_{4}^{2} a^{\prime}(t)^{2} \csc ^{8}(\eta)}{\alpha^{14} Q_{\alpha, 4}^{8}}+\frac{645120 \cot ^{2}(\eta) \xi_{1}^{2} \xi_{2}^{2} \xi_{3}^{8} a^{\prime}(t)^{2} \csc ^{8}(\eta)}{\alpha^{14} Q_{\alpha, 4}^{9}}+ \\
& \frac{1290240 \xi_{1}^{2} \xi_{2}^{2} \xi_{3}^{6} \xi_{a^{2}}^{2}(t)^{2} \csc ^{8}(\eta)}{\alpha^{14} Q_{\alpha, 4}^{9}}+\frac{112 \xi_{3}^{4} \csc ^{8}(\eta)}{\alpha^{8} Q_{\alpha, 4}^{5}}-\frac{1664 \cot ^{2}(\eta) \xi_{3}^{6} \csc ^{8}(\eta)}{\alpha^{10} Q_{\alpha, 4}^{6}}-\frac{1664 \xi_{2}^{2} \xi_{3}^{4} \csc ^{8}(\eta)}{\alpha^{10} Q_{\alpha, 4}^{6}}+ \\
& \frac{2560 \cot ^{4}(\eta) \xi_{3}^{8} \csc ^{8}(\eta)}{\alpha^{12} Q^{7}}+\frac{30080 \cot ^{2}(\eta) \xi_{2}^{2} \xi_{3}^{6} \csc ^{8}(\eta)}{\alpha^{12} Q^{7}}+\frac{2560 \xi_{2}^{4} \xi_{3}^{4} \csc ^{8}(\eta)}{\alpha^{12} Q_{\alpha, 4}^{7}}-\frac{10240 \xi_{3}^{6} \xi_{4}^{2} \csc ^{8}(\eta)}{\alpha^{12} Q_{\alpha}^{7}}- \\
& \frac{53760 \cot ^{4}(\eta) \xi_{2}^{2} \xi_{3}^{8} \csc ^{8}(\eta)}{\alpha^{14} Q_{\alpha, 4}^{8}}-\frac{53760 \cot ^{2}(\eta) \xi_{2}^{4} \xi_{3}^{6} \csc ^{8}(\eta)}{\alpha^{14} Q_{\alpha, 4}^{8}}+\frac{215040 \xi_{2}^{2} \xi_{3}^{6} \xi_{4}^{2} \csc ^{8}(\eta)}{\alpha^{14} Q_{\alpha, 4}^{8}}+\frac{107520 \cot ^{4}(\eta) \xi_{2}^{4} \xi_{3}^{8} \csc ^{8}(\eta)}{\alpha^{16} Q_{\alpha, 4}^{9}}- \\
& \frac{430080 \xi_{2}^{4} \xi_{3}^{6} \xi_{4}^{2} \csc ^{8}(\eta)}{\alpha^{16} Q_{\alpha, 4}^{9}}-\frac{2752 \xi_{3}^{6} a^{\prime}(t)^{4} \csc ^{6}(\eta)}{\alpha^{10} Q_{\alpha, 4}^{6}}+\frac{47040 \xi_{1}^{2} \xi_{3}^{6} a^{\prime}(t)^{4} \csc ^{6}(\eta)}{\alpha^{10} Q_{\alpha, 4}^{7}}+\cdots .
\end{aligned}
$$

Since the full expression for $b_{-6}$ is quite lengthy, we have recorded it in Appendix A.

In order to express $b_{-6}$ as a rational function, we introduce the following coordinates:

$$
\begin{gathered}
u_{0}=\sin ^{2}(\eta), \quad u_{1}=\xi_{1}, \quad u_{2}=\xi_{2}, \\
u_{3}=\csc (\eta) \xi_{3}, \quad u_{4}=\sec (\eta) \xi_{4}, \quad u_{5}=\xi_{5}, \quad u_{6}=\xi_{6} .
\end{gathered}
$$

First, let us note that in the new coordinates we have

$$
\begin{aligned}
& \tilde{\sigma}_{5}:=d \eta \wedge \sigma_{\xi, 5} \\
& =\sum_{j=1}^{6}(-1)^{j-1} \xi_{j} d \eta \wedge d \xi_{1} \wedge \cdots \wedge \widehat{d \xi_{j}} \wedge \cdots \wedge d \xi_{6} \\
& =\frac{1}{2}\left(u_{1} d u_{0} d u_{2} d u_{3} d u_{4} d u_{5} d u_{6}-u_{2} d u_{0} d u_{1} d u_{3} d u_{4} d u_{5} d u_{6}\right. \\
& +u_{3} d u_{0} d u_{1} d u_{2} d u_{4} d u_{5} d u_{6}-u_{4} d u_{0} d u_{1} d u_{2} d u_{3} d u_{5} d u_{6} \\
& \left.+u_{5} d u_{0} d u_{1} d u_{2} d u_{3} d u_{4} d u_{6}-u_{6} d u_{0} d u_{1} d u_{2} d u_{3} d u_{4} d u_{5}\right) \text {. }
\end{aligned}
$$

Finally, the rationality of $b_{-6}$ in the new coordinates (5.5) follows from the fact that the exponents of the $\xi_{j}$ appearing in the numerators in the expression of $b_{-6}$ are non-negative even integers, and all trigonometric terms in the expression, which are listed below, transform to the following rational expressions in the $u_{j}$ :

$$
\begin{gathered}
\xi_{1}^{2}+\frac{\xi_{2}^{2}}{a(t)^{2}}+\frac{\xi_{3}^{2} \csc ^{2}(\eta)}{a(t)^{2}}+\frac{\xi_{4}^{2} \sec ^{2}(\eta)}{a(t)^{2}}+\xi_{5}^{2}+\xi_{6}^{2}=u_{1}^{2}+\frac{1}{a(t)^{2}}\left(u_{2}^{2}+u_{3}^{2}+u_{4}^{2}\right)+u_{5}^{2}+u_{6}^{2}, \\
\cot ^{2}(\eta)=\frac{1-u_{0}}{u_{0}},
\end{gathered}
$$




$$
\begin{aligned}
& \csc ^{2}(\eta)=\frac{1}{u_{0}} \\
& \sec ^{2}(\eta)=\frac{1}{1-u_{0}} \\
& \cot ^{3}(\eta) \cot (2 \eta)=\frac{\cot ^{4}(\eta)}{8}-\frac{3 \cot ^{2}(\eta)}{4}+\frac{\csc ^{4}(\eta)}{8}-\frac{\csc ^{2}(\eta)}{4}+\frac{1}{4} \cot ^{2}(\eta) \csc ^{2}(\eta)+\frac{1}{8} \\
& \cot (2 \eta) \csc ^{5}(\eta) \sec (\eta)=\frac{\csc ^{6}(\eta)}{2}-\frac{1}{2} \csc ^{4}(\eta) \sec ^{2}(\eta), \\
& \cot (2 \eta) \csc (\eta) \sec ^{5}(\eta)=\frac{1}{2} \csc ^{2}(\eta) \sec ^{4}(\eta)-\frac{\sec ^{6}(\eta)}{2} \\
& \cot ^{2}(2 \eta)=\frac{\tan ^{2}(\eta)}{8}+\frac{\cot ^{2}(\eta)}{8}+\frac{1}{8} \csc ^{2}(\eta) \sec ^{2}(\eta)-\frac{3}{4}, \\
& \cot (\eta) \cot (2 \eta)=\frac{\cot ^{2}(\eta)}{2}-\frac{1}{2} \\
& \cot (2 \eta) \csc ^{3}(\eta) \sec (\eta)=\frac{\csc ^{4}(\eta)}{2}-\frac{1}{2} \csc ^{2}(\eta) \sec ^{2}(\eta) \\
& \csc ^{2}(2 \eta)=\frac{1}{4} \csc ^{2}(\eta) \sec ^{2}(\eta) \\
& \cot (2 \eta) \csc (\eta) \sec ^{3}(\eta)=\frac{1}{2} \csc ^{2}(\eta) \sec ^{2}(\eta)-\frac{\sec ^{4}(\eta)}{2} \\
& \tan (\eta) \cot (2 \eta)=\frac{1}{2}-\frac{\tan ^{2}(\eta)}{2} \\
& \tan (\eta) \cot ^{3}(2 \eta)= \\
& -\frac{\tan ^{4}(\eta)}{32}+\frac{15 \tan ^{2}(\eta)}{32}+\frac{\cot ^{2}(\eta)}{32}-\frac{1}{32} 3 \sec ^{4}(\eta)+\frac{3}{32} \csc ^{2}(\eta) \sec ^{2}(\eta)-\frac{15}{32} .
\end{aligned}
$$

We note that, in the case of $a_{2}$, the rational expression has $a(t), a^{\prime}(t), a^{\prime \prime}(t)$ appearing in the numerators, while in the case of $a_{4}$ we have $a(t), a^{\prime}(t), a^{\prime \prime}(t), a^{(3)}(t), a^{(4)}(t)$ appearing in the numerators. As in the $a_{2}$ case, we treat these as variables $\left(\alpha_{1}, \ldots, \alpha_{4}\right)$ $\in \mathbb{A}^{4}$. Since the denominators do not depend on the variables $\left(\alpha_{1}, \ldots, \alpha_{4}\right)$, we can regard the forms $\Omega^{\alpha}$ as a four-parameter family of algebraic differential forms defined over the same algebraic variety, given by the complement in the affine space $\mathbb{A}^{7}$ of the union of the hyperplanes $H_{0}$ and $H_{1}$ and the variety $\widehat{C Z}_{\alpha}$ determined by the vanishing of the quadratic form

$$
Q_{\alpha, 4}=u_{1}^{2}+\alpha^{-2}\left(u_{2}^{2}+u_{3}^{2}+u_{4}^{2}\right)+u_{5}^{2}+u_{6}^{2} .
$$

We view $\widehat{C Z}_{\alpha}$ as the affine cone in $\mathbb{A}^{7}$ over the projective cone $C Z_{\alpha}$ in $\mathbb{P}^{6}$ of the quadric $Z_{\alpha}$ in $\mathbb{P}^{5}$ defined by the vanishing of $Q_{\alpha}$. 
Theorem 5.2. When computed without performing the time integration, the $a_{4}$ term in the heat kernel expansion of $D^{2}$ is a period integral

$$
C \cdot \int_{A} \Omega_{\left(\alpha_{1}, \alpha_{2}, \alpha_{3}, \alpha_{4}\right)}^{\alpha}
$$

with the algebraic differential forms $\Omega_{\left(\alpha_{1}, \alpha_{2}, \alpha_{3}, \alpha_{4}\right)}^{\alpha}$ as in Proposition 5.1, and with domain of integration the $\mathbb{Q}$-semialgebraic set

$$
A_{6}=\left\{\left(u_{0}, \ldots, u_{6}\right) \in \mathbb{A}^{7}(\mathbb{R}): \begin{array}{l}
u_{1}^{2}+u_{2}^{2}+u_{0} u_{3}^{2}+\left(1-u_{0}\right) u_{4}^{2}+u_{5}^{2}+u_{6}^{2}=1 \\
0<u_{i}<1, \quad i=0,1,2,5,6
\end{array}\right\}
$$

The coefficient $C$ is in $\mathbb{Q}\left[(2 \pi i)^{-1}\right]$. This integral is a period of the mixed motive

$$
\mathfrak{m}\left(\mathbb{A}^{7} \backslash\left(\widehat{C Z}_{\alpha} \cup H_{0} \cup H_{1}\right), \Sigma\right),
$$

where $\widehat{C Z}_{\alpha}$ is the hypersurface in $\mathbb{A}^{7}$ defined by the vanishing of the quadric $Q_{\alpha}$ of (5.3), and $\Sigma=\cup_{i, a} H_{i, a}$ is the divisor given by the union of the hyperplanes $H_{i, a}=$ $\left\{u_{i}=a\right\}$, with $i \in\{0,1,2,5,6\}$ and $a \in\{0,1\}$.

Proof. The term $a_{4}$ is computed as

$$
a_{4}=\frac{1}{2^{5} \pi^{5}} \operatorname{Res}\left(\Delta_{4}^{-1}\right) .
$$

Therefore, before performing the integration in the time variable $t$, we can write explicitly the expression for the term $a_{4}$ as

$$
\begin{aligned}
& a_{4}=\frac{1}{2^{5} \pi^{5}} \int_{0}^{\pi / 2} d \eta \int_{0}^{2 \pi} d \phi_{1} \int_{0}^{2 \pi} d \phi_{2} \int_{\mathbb{T}^{2}} d^{2} x^{\prime} \int_{\xi_{1}^{2}+\xi_{2}^{2}+\xi_{3}^{2}+\xi_{4}^{2}+\xi_{5}^{2}+\xi_{6}^{2}=1} d^{5} \xi \cdot b_{-6} \cdot \sigma_{\xi, 5} \\
& =\frac{1}{2^{3} \pi^{3}} \int_{0}^{\pi / 2} d \eta \int_{\xi_{1}^{2}+\xi_{2}^{2}+\xi_{3}^{2}+\xi_{4}^{2}+\xi_{5}^{2}+\xi_{6}^{2}=1} d^{5} \xi \cdot b_{-6}\left(t, \eta, \xi_{1}, \ldots, \xi_{6}\right) \cdot \sigma_{\xi, 5} \\
& =\frac{1}{2^{3} \pi^{3}} \int_{\left(0, \frac{\pi}{2}\right) \times \mathbb{S}^{5}} b_{-6}\left(t, \eta, \xi_{1}, \ldots, \xi_{6}\right) \sum_{j=1}^{6}(-1)^{j-1} \xi_{j} d \eta \wedge d \xi_{1} \wedge \cdots \wedge \widehat{d}_{j} \wedge \cdots \wedge d \xi_{6} .
\end{aligned}
$$

As in Proposition 5.1, we perform the change of variables (5.5). In these coordinates the domain of integration $\left(0, \frac{\pi}{2}\right) \times \mathbb{S}^{5}$ in (5.9) transforms to the set

$A_{6}:=\left\{\left(u_{0}, \ldots, u_{6}\right) \in(0,1)^{3} \times \mathbb{R}^{2} \times(0,1)^{2} ; u_{1}^{2}+u_{2}^{2}+u_{0} u_{3}^{2}+\left(1-u_{0}\right) u_{4}^{2}+u_{5}^{2}+u_{6}^{2}=1\right\}$,

which is a $\mathbb{Q}$-semialgebraic set as in (5.7). The boundary $\partial A$ of the domain of integration is contained in the divisor $\Sigma=\cup_{i, a} H_{i, a}$, with $i \in\{0,1,2,5,6\}$ and $a \in$ $\{0,1\}$. For any fixed $\alpha \in \mathbb{G}_{m}$, and for any choice of $\left(\alpha_{1}, \ldots, \alpha_{4}\right) \in \mathbb{A}^{4}$, the forms $\Omega_{\left(\alpha_{1}, \alpha_{2}, \alpha_{3}, \alpha_{4}\right)}^{\alpha}$ are algebraic differential forms defined on the complement in $\mathbb{A}^{7}$ of the union of $\widehat{C Z}_{\alpha}$ and $H_{0} \cup H_{1}$, hence the integrals in (5.6) are periods of the motive $\mathfrak{m}\left(\mathbb{A}^{7} \backslash\left(\widehat{C Z}_{\alpha} \cup H_{0} \cup H_{1}\right), \Sigma\right)$. 
Again, as mentioned in Remark 4.3, although the singular set of the algebraic differential form intersects the divisor $\Sigma$, the integral is convergent, hence there is no renormalization issue involved.

\section{THE $a_{2 n}$ TERM}

We start by using (3.2) to express the term $a_{2 n}$ as the noncommutative residue of an operator, namely

$$
a_{2 n}=\frac{1}{2^{5} \pi^{3+n}} \operatorname{Res}\left(\Delta_{2 n}^{-1}\right)
$$

where

$$
\Delta_{2 n}=D^{2} \otimes 1+1 \otimes \Delta_{\mathbb{T}^{2 n-2}} .
$$

Here $\Delta_{\mathbb{T}^{2 n-2}}$ is the Laplacian of the flat metric on the torus of dimension $2 n-2$. Since $\Delta_{2 n}$ acts on the smooth sections of a vector bundle on a manifold of dimension $2 n+2$, in order to compute $\operatorname{Res}\left(\Delta_{2 n}^{-1}\right)$ one needs to calculate the term $\sigma_{-2 n-2}$ which is homogeneous of order $-2 n-2$ in the expansion of the pseudodifferential symbol of $\Delta_{2 n}^{-1}$.

Theorem 6.1. The term $\sigma_{-2 n-2}$ satisfies

$$
\begin{gathered}
\operatorname{tr}\left(\sigma_{-2 n-2}\right)= \\
\sum_{j=1}^{M_{n}} c_{j, 2 n} u_{0}^{\beta_{0,1, j} / 2}\left(1-u_{0}\right)^{\beta_{0,2, j} / 2} \frac{u_{1}^{\beta_{1, j}} u_{2}^{\beta_{2, j}} \cdots u_{2 n+2}^{\beta_{2 n+2, j}}}{Q_{\alpha, 2 n}^{\rho_{j, 2 n}}} \alpha^{k_{0, j}} \alpha_{1}^{k_{1, j}} \cdots \alpha_{2 n}^{k_{2 n, j}},
\end{gathered}
$$

where

$$
\begin{gathered}
\alpha=a(t), \quad \alpha_{1}=a^{\prime}(t), \quad \alpha_{2}=a^{\prime \prime}(t), \quad \ldots, \quad \alpha_{2 n}=a^{2 n}(t), \\
Q_{\alpha, 2 n}=u_{1}^{2}+\frac{1}{\alpha^{2}}\left(u_{2}^{2}+u_{3}^{2}+u_{4}^{2}\right)+u_{5}^{2}+\cdots+u_{2 n+2}^{2}, \\
c_{j, 2 n} \in \mathbb{Q}, \quad \beta_{0,1, j}, \beta_{0,2, j}, k_{0, j} \in \mathbb{Z}, \quad \beta_{1, j}, \ldots, \beta_{2 n+2, j}, \rho_{j, 2 n}, k_{1, j}, \ldots, k_{2 n, j} \in \mathbb{Z}_{\geq 0} .
\end{gathered}
$$

Proof. The argument is similar to our treatment of the terms $a_{2}$ and $a_{4}$. In order to finally see rational differential forms that integrate to the term $a_{2 n}$, it will be useful to perform calculations in the following coordinates:

$$
\begin{gathered}
u_{0}=\sin ^{2}(\eta), \quad u_{3}=\csc (\eta) \xi_{3}, \quad u_{4}=\sec (\eta) \xi_{4}, \\
u_{j}=\xi_{j}, \quad j=1,2,5,6, \ldots, 2 n+2 .
\end{gathered}
$$

In this regard, we use the new coordinates for writing the pseudodifferential symbol of the Dirac operator $D$ of the Robertson-Walker metric, namely that we rewrite

$$
\sigma(D)=q_{1}+q_{0}
$$


where $q_{1}$ and $q_{0}$ are now expressed as

$$
\begin{gathered}
q_{1}=\left(\begin{array}{cccc}
0 & 0 & \frac{i u_{4}}{a(t)}-u_{1} & \frac{i u_{2}}{a(t)}+\frac{u_{3}}{a(t)} \\
0 & 0 & \frac{i u_{2}}{a(t)}-\frac{u_{3}}{a(t)} & -u_{1}-\frac{i u_{4}}{a(t)} \\
-u_{1}-\frac{i u_{4}}{a(t)} & -\frac{i u_{2}}{a(t)}-\frac{u_{3}}{a(t)} & 0 & 0 \\
\frac{u_{3}}{a(t)}-\frac{i u_{2}}{a(t)} & \frac{i u_{4}}{a(t)}-u_{1} & 0 & 0
\end{array}\right), \\
q_{0}=\left(\begin{array}{cccc}
0 & \frac{3 i a^{\prime}(t)}{2 a(t)} & \frac{1-2 u_{0}}{2 a(t) \sqrt{\left(1-u_{0}\right) u_{0}}} \\
0 & 0 & \frac{1-2 u_{0}}{2 a(t) \sqrt{\left(1-u_{0}\right) u_{0}}} & \frac{3 i a^{\prime}(t)}{2 a(t)} \\
\frac{3 i a^{\prime}(t)}{2 a(t)} & -\frac{1-2 u_{0}}{2 a(t) \sqrt{\left(1-u_{0}\right) u_{0}}} & 0 & 0 \\
-\frac{1-2 u_{0}}{2 a(t) \sqrt{\left(1-u_{0}\right) u_{0}}} & \frac{3 i a^{\prime}(t)}{2 a(t)} & 0 & 0
\end{array}\right) .
\end{gathered}
$$

Since $q_{1}$ and $q_{0}$ depend only on $t$ and $u_{0}$, or equivalently only on $t$ and $\eta$, for the symbol of $D^{2}$, we have

$$
\sigma\left(D^{2}\right)=p_{2}+p_{1}+p_{0}
$$

where

$$
\begin{aligned}
& p_{2}=q_{1}^{2}=\left(u_{1}^{2}+\frac{1}{a(t)^{2}}\left(u_{2}^{2}+u_{3}^{2}+u_{4}^{2}\right)\right) I_{4 \times 4}, \\
& p_{1}=q_{0} q_{1}+q_{1} q_{0}+\left(-i \frac{\partial q_{1}}{\partial \xi_{1}} \frac{\partial q_{1}}{\partial t}-i \frac{\partial q_{1}}{\partial \xi_{2}} \frac{\partial q_{1}}{\partial \eta}\right) \\
& p_{0}=q_{0}^{2}+\left(-i \frac{\partial q_{1}}{\partial \xi_{1}} \frac{\partial q_{0}}{\partial t}-i \frac{\partial q_{1}}{\partial \xi_{2}} \frac{\partial q_{0}}{\partial \eta}\right) .
\end{aligned}
$$

It is possible to keep track of the general form of the expressions that will appear in the calculations, when written in the new coordinates given by (6.3), as follows. We achieve this by using the fact that if, for a smooth function $f$ of our variables, we use the notation

$$
f\left(t, \eta, \xi_{1}, \xi_{2}, \ldots, \xi_{2 n+2}\right)=\tilde{f}\left(t, u_{0}, u_{1}, u_{2}, \ldots, u_{2 n+2}\right),
$$

then

$$
\begin{aligned}
& \partial_{t} f=\partial_{t} \tilde{f}, \quad \partial_{\xi_{j}} f=\partial_{u_{j}} \tilde{f}, \quad j=1,2, \\
& \partial_{\eta} f=2 \sqrt{u_{0}\left(1-u_{0}\right)} \partial_{u_{0}} \tilde{f}-u_{3} \sqrt{\frac{1-u_{0}}{u_{0}}} \partial_{u_{3}} \tilde{f}+u_{4} \sqrt{\frac{u_{0}}{1-u_{0}}} \partial_{u_{4}} \tilde{f} .
\end{aligned}
$$

We can now focus on the homogeneous terms $\sigma_{-2-j}\left(\Delta_{2 n}^{-1}\right)$ of order $-2-j$ in the expansion of the pseudodifferential symbol of the parametrix of $\Delta_{2 n}$. As mentioned earlier, the relevant term for the calculation of $a_{2 n}$ is the term $\sigma_{-2 n-2}\left(\Delta_{2 n}^{-1}\right)$. By using (6.8) and considering the independence of the symbols from the variables $\phi_{1}$ and $\phi_{2}$, we have

$$
\sigma_{-2}\left(\Delta_{2 n}^{-1}\right)=\left(p_{2}+\left(u_{5}^{2}+\cdots+u_{2 n+2}^{2}\right) I_{4 \times 4}\right)^{-1}=\frac{1}{Q_{\alpha, 2 n}} I_{4 \times 4}
$$


where

$$
Q_{\alpha, 2 n}=u_{1}^{2}+\frac{1}{\alpha^{2}}\left(u_{2}^{2}+u_{3}^{2}+u_{4}^{2}\right)+u_{5}^{2}+\cdots+u_{2 n+2}^{2}
$$

and the desired $\sigma_{-2 n-2}\left(\Delta_{2 n}^{-1}\right)$ can be calculated recursively:

$$
\sigma_{-2 n-2}\left(\Delta_{2 n}^{-1}\right)=
$$

$$
-\left(\sum_{\substack{0 \leq j<2 n, 0 \leq k \leq 2 \\ \ell_{1}, \ell_{2} \in \mathbb{Z}_{\geq 0} \\-2-j-|\alpha|+k=-2 n}} \frac{(-i)^{\ell_{1}+\ell_{2}}}{\ell_{1} ! \ell_{2} !}\left(\partial_{\xi_{1}}^{\ell_{1}} \partial_{\xi_{2}}^{\ell_{2}} \sigma_{-2-j}\left(\Delta_{2 n}^{-1}\right)\right)\left(\partial_{t}^{\ell_{1}} \partial_{\eta}^{\ell_{2}} p_{k}\right)\right) \sigma_{-2}\left(\Delta_{2 n}^{-1}\right) .
$$

Combining these formulas with the equations given by (6.4), one can see by induction that (6.2) holds as stated.

Theorem 6.2. When computed without performing the time integration, the coefficient $a_{2 n}$ is a period integral

$$
C \cdot \int_{A_{2 n}} \Omega_{\alpha_{1}, \ldots, \alpha_{2 n}}^{\alpha}
$$

of an algebraic differential form $\Omega_{\alpha_{1}, \ldots, \alpha_{2 n}}^{\alpha}\left(u_{0}, u_{1}, \ldots, u_{2 n+2}\right)$ defined on the complement $\mathbb{A}^{2 n+3} \backslash\left(\widehat{C Z}_{\alpha, 2 n} \cup H_{0} \cup H_{1}\right)$, with $\widehat{C Z}_{\alpha, 2 n}$ the hypersurface defined by the vanishing of the quadric $Q_{\alpha, 2 n}$ of (6.6), and hyperplanes $H_{0}=\left\{u_{0}=0\right\}$ and $H_{1}=\left\{u_{0}=1\right\}$. The period integral is performed over the $\mathbb{Q}$-semialgebraic set

$$
A_{2 n+2}=
$$

$$
\left\{\left(u_{0}, \ldots, u_{2 n+2}\right) \in \mathbb{A}^{2 n+3}(\mathbb{R}): \begin{array}{l}
u_{1}^{2}+u_{2}^{2}+u_{0} u_{3}^{2}+\left(1-u_{0}\right) u_{4}^{2}+\sum_{i=5}^{2 n+2} u_{i}^{2}=1 \\
0<u_{i}<1, \quad i=0,1,2,5,6, \ldots, 2 n+2
\end{array}\right\} .
$$

Proof. Having obtained in Proposition 6.1 a general form for $\operatorname{tr}\left(\sigma_{-2 n-2}\right)$, we use the explicit definition of the Wodzicki residue, while leaving out an integration with respect to $t$, to expand the formula (6.1) for the term $a_{2 n}$ and we write:

$$
\begin{aligned}
& =\frac{1}{2^{5} \pi^{3+n}} \int_{0}^{\pi / 2} d \eta \int_{0}^{2 \pi} d \phi_{1} \int_{0}^{2 \pi} d \phi_{2} \int_{\mathbb{T}^{2 n-2}} d x^{\prime} \int_{\sum_{j=1}^{2 n+2} \xi_{j}^{2}=1} d \xi \cdot \operatorname{tr}\left(\sigma_{-2 n-2}\right) \cdot \sigma_{\xi, 2 n+1} \\
& =\frac{1}{2^{3} \pi^{1+n}} \int_{0}^{\pi / 2} d \eta \int_{\sum_{j=1}^{2 n+2} \xi_{j}^{2}=1} d \xi \cdot \operatorname{tr}\left(\sigma_{-2 n-2}\right) \cdot \sigma_{\xi, 2 n+1}
\end{aligned}
$$




$$
\begin{aligned}
& =\frac{1}{2^{3} \pi^{1+n}} \int_{\left(0, \frac{\pi}{2}\right) \times \mathbb{S}^{2 n+1}} \operatorname{tr}\left(\sigma_{-2 n-2}\right) \sum_{j=1}^{2 n+2}(-1)^{j-1} \xi_{j} d \eta \wedge d \xi_{1} \wedge \cdots \wedge \widehat{d}_{j} \wedge \cdots \wedge d \xi_{2 n+2} \\
& =\frac{1}{2^{3} \pi^{1+n}} \int_{(\eta, \xi) \in\left(0, \frac{\pi}{2}\right) \times \mathbb{S}^{2 n+1}} \operatorname{tr}\left(\sigma_{-2 n-2}\right) \widetilde{\sigma}_{2 n+1},
\end{aligned}
$$

where

$$
\begin{aligned}
& \widetilde{\sigma}_{2 n+1}:=d \eta \wedge \sigma_{\xi, 2 n+1} \\
& =\sum_{j=1}^{2 n+2}(-1)^{j-1} \xi_{j} d \eta \wedge d \xi_{1} \wedge \cdots \wedge \widehat{d} \xi_{j} \wedge \cdots \wedge d \xi_{2 n+2} \\
& =\frac{1}{2} \sum_{j=1}^{2 n+2}(-1)^{j-1} u_{j} d u_{0} d u_{1} \wedge \cdots \wedge \widehat{d} u_{j} \wedge \cdots \wedge d u_{2 n+2} .
\end{aligned}
$$

Considering the general form of $\operatorname{tr}\left(\sigma_{-2 n-2}\right)$ given by (6.2), which is a rational expression in $\sqrt{u_{0}}, \sqrt{1-u_{0}}, u_{1}, \ldots, u_{2 n+2}, \alpha, \alpha_{1}, \ldots, \alpha_{2 n}$, in order to prove that $a_{2 n}$ is the integral of a rational differential form, our next task is apparently to argue that only the terms that have even powers of $\sqrt{u_{0}}$ and $\sqrt{1-u_{0}}$ involved contribute to the calculation of the term $a_{2 n}$. This will in particular show that the last integral expression given for $a_{2 n}$ by (6.9) is equal to the integral of a rational differential form in $u_{0}, u_{1}, \ldots, u_{2 n+2}, \alpha, \alpha_{1}, \ldots, \alpha_{2 n}$ over the $\mathbb{Q}$-semialgebraic set (6.8).

The claim that only the terms with $\beta_{0,1, j}, \beta_{0,2, j} \in 2 \mathbb{Z}$ in the summation given by (6.2) contribute to the calculation of the term $a_{2 n}$ can be proved as follows. Fixing a point $\left(x, x^{\prime}\right)=\left(t, \eta, \phi_{1}, \phi_{2}, x^{\prime}\right) \in M \times \mathbb{T}^{2 n-2}$, the differential form $\operatorname{tr}\left(\sigma_{-2 n-2}\right) \sigma_{\xi, 2 n+1}$ on the Euclidean space $\mathbb{R}^{2 n+2} \simeq T_{\left(x, x^{\prime}\right)}^{*}\left(M \times \mathbb{T}^{2 n-2}\right)$ is a closed differential form of degree $2 n+1$. The reason is that $\operatorname{tr}\left(\sigma_{-2 n-2}\right)$ is homogeneous of order $-2 n-2$ in $\xi \in \mathbb{R}^{2 n+2}$, cf. Proposition 7.3 on page 265 of [13]. Therefore, using the Stokes theorem, the integral of this differential form over the unit sphere $|\xi|=1$ is the same as its integral over the cosphere of the metric in the cotangent fibre given by

$$
\begin{aligned}
|\xi|_{g}^{2} & =\xi_{1}^{2}+\frac{\xi_{2}^{2}}{a(t)^{2}}+\frac{\csc ^{2}(\eta) \xi_{3}^{2}}{a(t)^{2}}+\frac{\sec ^{2}(\eta) \xi_{4}^{2}}{a(t)^{2}}+\xi_{5}^{2}+\cdots+\xi_{2 n+2}^{2} \\
& =u_{1}^{2}+\frac{1}{\alpha^{2}}\left(u_{2}^{2}+u_{3}^{2}+u_{4}^{2}\right)+u_{5}^{2}+\cdots+u_{2 n+2}^{2} \\
& =1 .
\end{aligned}
$$

We parametrize the cosphere $|\xi|_{g}=1$ by writing

$$
\begin{aligned}
& \xi_{1}=\sin \left(\psi_{2 n+1}\right) \sin \left(\psi_{2 n}\right) \cdots \sin \left(\psi_{2}\right) \cos \left(\psi_{1}\right), \\
& \xi_{2}=\alpha \sin \left(\psi_{2 n+1}\right) \sin \left(\psi_{2 n}\right) \cdots \sin \left(\psi_{2}\right) \sin \left(\psi_{1}\right), \\
& \xi_{3}=\frac{\alpha}{\csc (\eta)} \sin \left(\psi_{2 n+1}\right) \sin \left(\psi_{2 n}\right) \cdots \sin \left(\psi_{3}\right) \cos \left(\psi_{2}\right), \\
& \xi_{4}=\frac{\alpha}{\sec (\eta)} \sin \left(\psi_{2 n+1}\right) \sin \left(\psi_{2 n}\right) \cdots \sin \left(\psi_{4}\right) \cos \left(\psi_{3}\right),
\end{aligned}
$$




$$
\begin{aligned}
\xi_{5} & =\sin \left(\psi_{2 n+1}\right) \sin \left(\psi_{2 n}\right) \cdots \sin \left(\psi_{5}\right) \cos \left(\psi_{4}\right), \\
\xi_{6} & =\sin \left(\psi_{2 n+1}\right) \sin \left(\psi_{2 n}\right) \cdots \sin \left(\psi_{6}\right) \cos \left(\psi_{5}\right), \\
& \cdots \\
\xi_{2 n+1} & =\sin \left(\psi_{2 n+1}\right) \cos \left(\psi_{2 n}\right), \\
\xi_{2 n+2} & =\cos \left(\psi_{2 n+1}\right),
\end{aligned}
$$

with the variables $\psi_{1}, \ldots, \psi_{2 n+1}$ having the following ranges:

$$
0<\psi_{1}<2 \pi, \quad 0<\psi_{2}<\pi, \quad 0<\psi_{3}<\pi, \quad \ldots, \quad 0<\psi_{2 n+1}<\pi .
$$

Using this parametrization, over the cosphere $|\xi|_{g}=1$, we have

$$
\begin{aligned}
\sigma_{\xi, 2 n+1} & =\sum_{j=1}^{2 n+2}(-1)^{j-1} \xi_{j} d \xi_{1} \wedge \cdots \wedge \widehat{d}_{j} \wedge \cdots \wedge d \xi_{2 n+2} \\
& =\alpha^{3} \sin (\eta) \cos (\eta) \sin \left(\psi_{2}\right) \sin ^{2}\left(\psi_{3}\right) \cdots \sin ^{2 n}\left(\psi_{2 n+1}\right) d \psi_{1} d \psi_{2} \cdots d \psi_{2 n+1} .
\end{aligned}
$$

Combining this form with the expression given by (6.2), we conclude that over $|\xi|_{g}=1$ we have:

$$
\begin{gathered}
\operatorname{tr}\left(\sigma_{-2 n-2}\right) \sigma_{\xi, 2 n+1}= \\
\sin (\eta) \cos (\eta) \sum_{j=1}^{M_{n}}\left\{c_{j, 2 n} \alpha^{\beta_{2, j}+\beta_{3, j}+\beta_{4, j}+k_{0, j}} \alpha_{1}^{k_{1, j}} \cdots \alpha_{2 n}^{k_{2 n, j}} \sin ^{\beta_{0,1, j}}(\eta) \cos ^{\beta_{0,2, j}}(\eta)\right. \\
\left.\cos ^{\beta_{1, j}}\left(\psi_{1}\right) \sin ^{\beta_{2, j}}\left(\psi_{1}\right) \prod_{\ell=2}^{2 n+1}\left(\sin \psi_{\ell}\right)^{\ell-1+\sum_{i=1}^{\ell} \beta_{i, j}}\left(\cos \psi_{\ell}\right)^{\beta_{\ell+1, j}}\right\} d \psi_{1} d \psi_{2} \cdots d \psi_{2 n+1} .
\end{gathered}
$$

By exploiting symmetries of the Robertson-Walker metric and its consequent rich isometry group, it is shown in Lemma 1 of [10] that the local invariant that integrates to the term $a_{2 n}$ has a spatial independence. This in particular means that the following expression is independent of the variable $\eta$ :

$$
\begin{aligned}
& \frac{1}{\sin (\eta) \cos (\eta)} \int_{|\xi|_{g}=1} \operatorname{tr}\left(\sigma_{-2 n-2}\right) \sigma_{\xi, 2 n+1} \\
= & \sum_{j=1}^{M_{n}} c_{j, 2 n} d_{j, 2 n} \alpha^{\beta_{2, j}+\beta_{3, j}+\beta_{4, j}+k_{0, j}} \alpha_{1}^{k_{1, j}} \cdots \alpha_{2 n}^{k_{2 n, j}} \sin ^{\beta_{0,1, j}}(\eta) \cos ^{\beta_{0,2, j}}(\eta),
\end{aligned}
$$

where

$$
\begin{aligned}
d_{j, 2 n}= & \int_{0}^{2 \pi} \cos ^{\beta_{1, j}}\left(\psi_{1}\right) \sin ^{\beta_{2, j}}\left(\psi_{1}\right) d \psi_{1} \times \\
& \int_{0}^{\pi} d \psi_{2} \cdots \int_{0}^{\pi} d \psi_{2 n+1} \prod_{\ell=2}^{2 n+1}\left(\sin \psi_{\ell}\right)^{\ell-1+\sum_{i=1}^{\ell} \beta_{i, j}}\left(\cos \psi_{\ell}\right)^{\beta_{\ell+1, j}} .
\end{aligned}
$$

We now exploit the independence from $\eta$ of the sum given by (6.11) to show that only the terms in (6.2) for which $\beta_{0,1, j}$ and $\beta_{0,2, j}$ are both even integers contribute to the calculation of the heat coefficient $a_{2 n}$. This follows easily from the fact that if 
for some coefficients $c_{j}$ and some integers $\gamma_{j}$ and $\nu_{j}$, a finite summation of the form $\sum_{j} c_{j} \sin ^{\gamma_{j}}(\eta) \cos ^{\nu_{j}}(\eta)$ is identically equal to a non-zero constant, or without loss in generality equal to 1 , then all the exponents $\gamma_{j}$ and $\nu_{j}$ are even integers in the sense that possible terms with odd exponents involved have to inevitably cancel each other out. Since $\eta$ varies between 0 and $\pi / 2$, this is equivalent to saying that if

$$
\sum_{j} c_{j} s^{\gamma_{j}}\left(1-s^{2}\right)^{\nu_{j} / 2}=1, \quad s \in(0,1)
$$

then all $\gamma_{j}$ and $\nu_{j}$ are even integers in the mentioned sense. It is useful to point out that a simple replacement of $s$ in the above equation by $s_{1}=\left(1-s^{2}\right)^{1 / 2}$ shows that our claim is symmetric with respect to the $\gamma_{j}$ and $\nu_{j}$ (therefore it suffices to argue that all $\gamma_{j}$ are even integers). We decompose the summation on the left side of the above identity and write

$$
\sum_{j_{o}} c_{j_{o}} s^{\gamma_{j_{o}}}\left(1-s^{2}\right)^{\nu_{j_{o}} / 2}+\sum_{j_{e}} c_{j_{e}} s^{\gamma_{j_{e}}}\left(1-s^{2}\right)^{\nu_{j_{e}} / 2}=1, \quad s \in(0,1)
$$

where for each term in the first summation either $\gamma_{j_{o}}$ or $\nu_{j_{o}}$ is odd, and in the second summation the $\gamma_{j_{e}}$ and $\nu_{j_{e}}$ are even integers. Therefore we have

$$
\sum_{j_{o}} c_{j_{o}} s^{\gamma_{j_{o}}}\left(1-s^{2}\right)^{\nu_{j_{o}} / 2}=1-\sum_{j_{e}} c_{j_{e}} s^{\gamma_{j_{e}}}\left(1-s^{2}\right)^{\nu_{j_{e}} / 2}, \quad s \in(0,1)
$$

and we proceed by considering the binomial series of the two sides of this equation. Since the series of the right hand side has only even powers of the variable $s \in(0,1)$, it follows that the terms on left side whose $\gamma_{j_{o}}$ are odd cancel each other out, therefore with no loss in generality we can assume all the $\gamma_{j_{o}}$ are even, which implies that all the $\nu_{j_{o}}$ have to be odd integers. Now by making the replacement $s_{1}=\left(1-s^{2}\right)^{1 / 2}$ we are led to

$$
\sum_{j_{o}} c_{j_{o}}\left(1-s_{1}^{2}\right)^{\gamma_{j_{o}} / 2} s_{1}^{\nu_{j_{o}}}=1-\sum_{j_{e}} c_{j_{e}}\left(1-s_{1}^{2}\right)^{\gamma_{j_{e}} / 2} s_{1}^{\nu_{j_{e}}}, \quad s_{1} \in(0,1) .
$$

Finally we compare the binomial series in $s_{1}$ of the two sides of this equation: since the series of the right hand side has only even exponents and all the $\nu_{j_{o}}$ on the left side are odd integers, we conclude that

$$
\sum_{j_{o}} c_{j_{o}}\left(1-s_{1}^{2}\right)^{\gamma_{j_{o}} / 2} s_{1}^{\nu_{j_{o}}}=0, \quad s_{1} \in(0,1)
$$

Again, as mentioned in Remark 4.3, the integrals are all convergent, hence there is no renormalization problem caused by the intersection of the boundary of the domain of integration with the singular set of the algebraic differential form. 


\section{The Motives}

In this section we analyze the motives associated to the periods obtained from the coefficients $a_{2 n}$ of the spectral action. We are considering a family of quadrics

$$
Q_{\alpha, 2 n}=u_{1}^{2}+\frac{1}{\alpha^{2}}\left(u_{2}^{2}+u_{3}^{2}+u_{4}^{2}\right)+u_{5}^{2}+\cdots+u_{2 n+2}^{2},
$$

where $\alpha$ is a (rational) parameter. These define quadric hypersurfaces $Z_{\alpha, 2 n}$ in $\mathbb{P}^{2 n+1}$. We will also be considering the projective cone $C Z_{\alpha, 2 n}$ in $\mathbb{P}^{2 n+2}$ and the affine cone $\widehat{C Z}_{\alpha, 2 n}$ in the affine space $\mathbb{A}^{2 n+3}$.

7.1. Pencils of quadrics. A quadratic form $Q$ on a vector space $V$ determines a quadric $Z_{Q} \subset \mathbb{P}(V)$. Given two quadratic forms $Q_{1}$ and $Q_{2}$ on $V$, a pencil $\mathcal{Z}_{\mathcal{Q}}$ of quadrics in $\mathbb{P}(V)$ is obtained by considering, for each $z=(\lambda: \mu) \in \mathbb{P}^{1}$, the quadric $Z_{Q_{z}}$ defined by the quadratic form $\lambda Q_{1}+\mu Q_{2}$. Let $\mathcal{Z}_{\mathcal{Q}}=\left\{(z, u) \in \mathbb{P}^{1} \times \mathbb{P}(V): u \in\right.$ $\left.Z_{Q_{z}}\right\} \subset \mathbb{P}^{1} \times \mathbb{P}(V)$.

In particular, we can view the quadrics $Z_{\alpha, 2 n}$ defined by the quadratic forms $Q_{\alpha, 2 n}$ of (17.1) as defining a pencil of quadrics in $\mathbb{P}^{1} \times \mathbb{P}^{2 n+1}$, with $\lambda / \mu=\alpha^{2}$. Namely, we regard the quadric $Z_{\alpha, 2 n}$ as part of the pencil of quadrics $\mathcal{Z}_{2 n}=\left\{Z_{z, 2 n}\right\}_{z \in \mathbb{P}^{1}}$, defined by

$$
Q_{z, 2 n}=\lambda\left(u_{1}^{2}+u_{5}^{2}+\cdots+u_{2 n+2}^{2}\right)+\mu\left(u_{2}^{2}+u_{3}^{2}+u_{4}^{2}\right),
$$

for $z=(\lambda: \mu) \in \mathbb{P}^{1}$. The quadric $Z_{z, 2 n}$ becomes degenerate over the set $X=\{0,1\} \subset$ $\mathbb{P}^{1}$, where it reduces, in the case $\lambda=0$ to a projective cone $Z_{Q_{1}, 2 n}=C^{2 n-1} B_{1}$ over the conic $B_{1}=\left\{u_{2}^{2}+u_{3}^{2}+u_{4}^{2}=0\right\}$ in $\mathbb{P}^{2}$, and in the case $\mu=0$ to a projective cone $Z_{Q_{2}, 2 n}=C^{3} B_{2}$ over the quadric $B_{2}=\left\{u_{1}^{2}+u_{5}^{2}+\cdots+u_{2 n+2}^{2}=0\right\}$ in $\mathbb{P}^{2 n-2}$. There is a correspondence, as in $\S 10$ of [2],

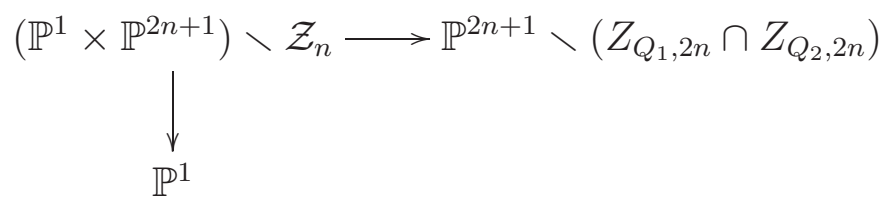

where the horizontal map is an $\mathbb{A}^{1}$-fibration and the vertical map is the projection to $z=(\lambda: \mu) \in \mathbb{P}^{1}$. By homotopy invariance, we can identify $H_{c}^{2 n+2}\left(\left(\mathbb{P}^{1} \times \mathbb{P}^{2 n+1}\right) \backslash \mathcal{Z}_{2 n}\right)$ with the Tate twisted $H_{c}^{2 n+1}\left(\mathbb{P}^{2 n+1} \backslash\left(Z_{Q_{1}, 2 n} \cap Z_{Q_{2}, 2 n}\right)\right)(-1)$.

7.2. Motives of quadrics. The theory of motives of quadrics is a very rich and interesting topic, see [16], [18, [19]. We recall here only a few essential facts that we need in our specific case. Suppose given a quadratic form $Q$ on an $n$-dimensional vector space $V$ over a field $\mathbb{K}$ of characteristic not equal to 2. For our purposes, we will focus on the case where $\mathbb{K}=\mathbb{Q}$. We write $\left\langle a_{1}, \ldots, a_{n}\right\rangle$ for the matrix of $Q$ in diagonal form. The quadratic form $\mathbb{H}:=\langle 1,-1\rangle$ is the elementary hyperbolic form. A quadratic form $Q$ is isotropic if $\mathbb{H}$ is a direct summand, hence $Q=\mathbb{H} \perp Q^{\prime}$. It is anisotropic otherwise. Any quadratic form can be written in the form $Q=d \cdot \mathbb{H} \perp Q^{\prime}$, where $Q^{\prime}$ is a uniquely determined anisotropic quadratic form. The integer $d$ is the 
Witt isotropy index of $Q$. Given an anisotropic quadratic form $Q$ over the field $\mathbb{K}$, there is a tower of field extensions $\mathbb{K}_{1}=\mathbb{K}(Q), \mathbb{K}_{2}=\mathbb{K}_{1}\left(Q_{1}\right), \ldots, \mathbb{K}_{s}=\mathbb{K}_{s-1}\left(Q_{s-1}\right)$, such that over $\mathbb{K}_{1}$ the quadric $\left.Q\right|_{\mathbb{K}_{1}}=d_{1} \cdot \mathbb{H} \perp Q_{1}$, with $Q_{1}$ anisotropic; over $\mathbb{K}_{2}$ the quadric $\left.Q_{1}\right|_{\mathbb{K}_{2}} d_{2} \cdot \mathbb{H} \perp Q_{2}$, with $Q_{2}$ anisotropic, and so on, until $Q_{s}=0$. The tower of extensions $\mathbb{K}_{1}, \ldots, \mathbb{K}_{s}$ is the Knebusch universal splitting tower, and $d_{1}, \ldots, d_{s}$ are the Witt numbers of $Q$.

Let $Z_{Q}$ be the quadric defined by the quadratic form $Q$ over $\mathbb{K}$. For a hyperbolic quadratic form $Q=d \cdot \mathbb{H}$ of dimension $2 d$, the motive of $Z_{Q}$ is given by (see [19])

$$
\mathfrak{m}\left(Z_{d \mathbb{H}}\right)=\mathbb{Z}(d-1)[2 d-2] \oplus \mathbb{Z}(d-1)[2 d-2] \oplus \bigoplus_{i=0, \ldots, d-2, d, \ldots, 2 d-2} \mathbb{Z}(i)[2 i],
$$

where $\mathbb{Z}=\mathfrak{m}(\operatorname{Spec}(\mathbb{K}))$. In the case where $Q=d \cdot \mathbb{H} \perp\langle 1\rangle$ in dimension $2 d+1$, the motive of $Z_{Q}$ is given by (see [19])

$$
\mathfrak{m}\left(Z_{d \mathbb{H} \perp\langle 1\rangle}\right)=\bigoplus_{i=0, \ldots, 2 d-1} \mathbb{Z}(i)[2 i] .
$$

Given a quadric $Z_{Q}$, we denote by $Z_{Q^{i}}$ the variety of $i$-dimensional planes on the quadric $Z_{Q}$. As in [19], we write $\mathcal{X}_{Q^{i}}$ for the associated simplicial scheme (Definition 2.3.1 of [19]) and $\mathfrak{m}\left(\mathcal{X}_{Q^{i}}\right)$ for the corresponding object in the category $\mathcal{D} \mathcal{M}^{\text {eff }}(\mathbb{K})$ of motives.

We also recall the following result (see Proposition 4.2 of [19]) that will be useful in our case. Let $Z_{Q} \subset \mathbb{P}^{m+1}$ be a quadratic form of dimension $m=2 n$ over $\mathbb{K}$, such that there exists a quadratic extension $\mathbb{K}(\sqrt{a})$ of $\mathbb{K}$ over which $Q$ is hyperbolic. Then the motive $\mathfrak{m}\left(Z_{Q}\right)$ decomposes as a direct sum

$$
\mathfrak{m}\left(Z_{Q}\right)=\left\{\begin{array}{lll}
\mathfrak{m}_{1} \oplus \mathfrak{m}_{1}(1)[2] & m=2 & \bmod 4 \\
\mathfrak{m}_{1} \oplus \mathcal{R}_{Q, \mathbb{K}} \oplus \mathfrak{m}_{1}(1)[2] & m=0 & \bmod 4
\end{array}\right.
$$

where the motive $\mathfrak{m}_{1}$ is an extension of the motives $\mathfrak{m}\left(\mathcal{X}_{Q^{i}}\right)(i)[2 i]$ and $\mathfrak{m}\left(\mathcal{X}_{Q^{\ell}}\right)(\operatorname{dim}(Q)$ $\ell)[2 \operatorname{dim}(Q)-2 \ell]$, for $i$ (respectively, $\ell$ ) ranging over all even (respectively, odd) numbers less than or equal to $2[\operatorname{dim}(Q) / 4]$. The motive we denote by $\mathcal{R}_{Q, \mathbb{K}}$ is a form of a Tate motive, which is denoted by $\mathcal{R}_{Q, \mathbb{K}}=\mathbb{K}(\sqrt{\operatorname{det}(Q)})\left(\frac{\operatorname{dim}(Q)}{2}\right)[\operatorname{dim}(Q)]$ in [19].

If $Q$ is $d$-times isotropic, $Q=d \cdot \mathbb{H} \perp Q^{\prime}$, then $\mathfrak{m}\left(\mathcal{X}_{Q^{j}}\right)=\mathbb{Z}$ for all $0 \leq j<i$. Thus, the motives $\mathfrak{m}\left(\mathcal{X}_{Q^{j}}\right)$ become Tate motives in a field extension in which the quadric becomes isotropic, and one recovers the motivic decomposition into a sum of Tate motives mentioned above. The motives $\mathfrak{m}\left(\mathcal{X}_{Q^{j}}\right)$ are therefore forms of the Tate motive, which means that over the algebraic closure $\mathfrak{m}\left(\left.\mathcal{X}_{Q^{j}}\right|_{\overline{\mathbb{K}}}\right)=\mathbb{Z}$.

7.3. Grothendieck classes. It if often convenient, instead of working with objects in the category of mixed motives, to consider a simpler invariant given by the class in the Grothendieck ring of varieties, which can be regarded as a universal Euler characteristics. The Grothendieck ring $K_{0}\left(\mathcal{V}_{\mathbb{K}}\right)$ of varieties over a field $\mathbb{K}$ is generated by the isomorphism classes $[X]$ of smooth quasi-projective varieties $X \in \mathcal{V}_{\mathbb{K}}$ with the inclusion-exclusion relations $[X]=[Y]+[X \backslash Y]$ for closed embeddings $Y \subset X$ 
and the product $[X \times Y]=[X] \cdot[Y]$. The following simple identities will be useful in the computations of Grothendieck classes of the motives involved in the period computations described in the previous sections.

Lemma 7.1. Let $Z$ be a projective subvariety $Z \subset \mathbb{P}^{N-1}$, with $\hat{Z} \subset \mathbb{A}^{N}$ the affine cone. Let $C Z$ denote the projective cone in $\mathbb{P}^{N}$ and $\widehat{C Z}$ the corresponding affine cone in $\mathbb{A}^{N+1}$. Let $H$ and $H^{\prime}$ be two affine hyperplanes in $\mathbb{A}^{N+1}$ with $H \cap H^{\prime}=\emptyset$ and such that the intersections $\widehat{C Z} \cap H$ and $\widehat{C Z} \cap H^{\prime}$ are sections of the cone, given by copies of $\hat{Z}$. The Grothendieck classes of the projective and affine complements satisfy

(1) $\left[\mathbb{A}^{N} \backslash \hat{Z}\right]=(\mathbb{L}-1)\left[\mathbb{P}^{N-1} \backslash Z\right]$

(2) $\left[\mathbb{A}^{N+1} \backslash \widehat{C Z}\right]=(\mathbb{L}-1)\left[\mathbb{P}^{N} \backslash C Z\right]$

(3) $[C Z]=\mathbb{L}[Z]+1$

(4) $\left[\mathbb{A}^{N+1} \backslash \widehat{C Z}\right]=\mathbb{L}^{N+1}-\mathbb{L}(\mathbb{L}-1)[Z]-\mathbb{L}$

(5) $\left[\mathbb{A}^{N+1} \backslash\left(\widehat{C Z} \cup H \cup H^{\prime}\right)\right]=\mathbb{L}^{N+1}-2 \mathbb{L}^{N}-(\mathbb{L}-2)(\mathbb{L}-1)[Z]-(\mathbb{L}-2)$.

where $\mathbb{L}=\left[\mathbb{A}^{1}\right]$ is the Lefschetz motive, the class of the affine line.

Proof. The first and second identities follow from the fact that the class of the affine cone is given by $[\hat{Z}]=(\mathbb{L}-1)[Z]+1$, so that

$$
\left[\mathbb{A}^{N} \backslash \hat{Z}\right]=\mathbb{L}^{N}-(\mathbb{L}-1)[Z]-1=(\mathbb{L}-1)\left(\frac{\left(\mathbb{L}^{N}-1\right)}{(\mathbb{L}-1)}-[Z]\right)=(\mathbb{L}-1)\left[\mathbb{P}^{N-1}-Z\right] .
$$

The identity $[C Z]=\mathbb{L}[Z]+1$ follows by viewing the projective cone over $Z$ as the union of a copy of $Z$ and a copy of the affine cone $\hat{Z}$ over $Z$, and using the same identity $[\hat{Z}]=(\mathbb{L}-1)[Z]+1$ for the affine cone. The fourth identity follows from the second and the third,

$$
\begin{gathered}
(\mathbb{L}-1)\left[\mathbb{P}^{N} \backslash C Z\right]=\mathbb{L}^{N+1}-1-(\mathbb{L}-1)[C Z] \\
=\mathbb{L}^{N+1}-1-(\mathbb{L}-1)(\mathbb{L}[Z]+1)=\mathbb{L}^{N+1}-\left(\mathbb{L}^{2}-\mathbb{L}\right)[Z]-\mathbb{L} .
\end{gathered}
$$

For the last identity, we write

$$
\left[\mathbb{A}^{N+1} \backslash\left(\widehat{C Z} \cup H \cup H^{\prime}\right)\right]=\mathbb{L}^{N+1}-\left[\widehat{C Z} \cup H \cup H^{\prime}\right] .
$$

The class of the union is given by

$$
\left[\widehat{C Z} \cup H \cup H^{\prime}\right]=[\widehat{C Z}]+\left[H \cup H^{\prime}\right]-\left[\widehat{C Z} \cap\left(H \cup H^{\prime}\right)\right]
$$

Since $H \cap H^{\prime}=\emptyset$, we have $\left[H \cup H^{\prime}\right]=2 \mathbb{L}^{N}$ and $\left[\widehat{C Z} \cap\left(H \cup H^{\prime}\right)\right]=[\widehat{C Z} \cap H]+[\widehat{C Z} \cap$ $\left.H^{\prime}\right]=2[\hat{Z}]=2(\mathbb{L}-1)[Z]+2$. Thus, we have

$$
\begin{gathered}
{\left[\mathbb{A}^{N+1} \backslash\left(\widehat{C Z} \cup H \cup H^{\prime}\right)\right]=\mathbb{L}^{N+1}-2 \mathbb{L}^{N}-[\widehat{C Z}]+2(\mathbb{L}-1)[Z]+2} \\
=\mathbb{L}^{N+1}-2 \mathbb{L}^{N}-\mathbb{L}(\mathbb{L}-1)[Z]-\mathbb{L}+2(\mathbb{L}-1)[Z]+2=\mathbb{L}^{N+1}-2 \mathbb{L}^{N}-(\mathbb{L}-2)(\mathbb{L}-1)[Z]-(\mathbb{L}-2) .
\end{gathered}
$$


7.4. Pencils of quadrics in $\mathbb{P}^{3}$. We look first at the case of the quadric $Z_{\alpha}=Z_{\alpha, 2}$ in $\mathbb{P}^{3}$ that arises in the computation of the $a_{2}$ term of the heat kernel expansion.

Over $\mathbb{C}$, any quadric surface $Z_{Q}$ in $\mathbb{P}^{3}$ can be put in the standard form $X Y=Z W$ by a simple change of coordinates. Thus, over $\mathbb{C}$ any quadric surface in $\mathbb{P}^{3}$ is isomorphic to the Segre embedding $\mathbb{P}^{1} \times \mathbb{P}^{1} \hookrightarrow \mathbb{P}^{3}$. When we consider quadrics over $\mathbb{Q}$, this is no longer necessarily the case.

Theorem 7.2. For $\alpha \in \mathbb{Q}$, over the quadratic extension $\mathbb{K}=\mathbb{Q}(\sqrt{-1})$, the quadric $Z_{\alpha}=Z_{\alpha, 2}$ in $\mathbb{P}^{3}$ is isomorphic to the Segre embedding $\mathbb{P}^{1} \times \mathbb{P}^{1} \hookrightarrow \mathbb{P}^{3}$. The class of the complement in the Grothendieck ring is $\left[\mathbb{P}^{3} \backslash Z_{\alpha}\right]=\mathbb{L}^{3}-\mathbb{L}$, while the class of the affine complement of $\widehat{C Z}_{\alpha}$ is $\left[\mathbb{A}^{5} \backslash \widehat{C Z}_{\alpha}\right]=\mathbb{L}^{5}-\mathbb{L}^{4}-\mathbb{L}^{3}+\mathbb{L}^{2}$. The class of the complement $\mathbb{A}^{5} \backslash\left(\widehat{C Z}_{\alpha} \cup H_{0} \cup H_{1}\right)$ with the affine hyperplanes $H_{0}=\left\{u_{0}=0\right\}$ and $H_{1}=\left\{u_{0}=1\right\}$ is given by

$$
\left[\mathbb{A}^{5} \backslash\left(\widehat{C Z}_{\alpha} \cup H_{0} \cup H_{1}\right)\right]=\mathbb{L}^{5}-3 \mathbb{L}^{4}+\mathbb{L}^{3}+3 \mathbb{L}^{2}-2 \mathbb{L} .
$$

Proof. Over the quadratic extension $\mathbb{K}=\mathbb{Q}(i)$ we can consider the change of variables

$$
X=u_{1}+\frac{i}{\alpha} u_{2}, \quad Y=u_{1}-\frac{i}{\alpha} u_{2}, \quad Z=\frac{i}{\alpha}\left(u_{3}+i u_{4}\right), \quad W=\frac{i}{\alpha}\left(u_{3}-i u_{4}\right),
$$

where we assume that $\alpha \in \mathbb{Q}$. This change of coordinates determines the identification of $Z_{\alpha}$ with the Segre quadric $\{X Y-Z W=0\} \simeq \mathbb{P}^{1} \times \mathbb{P}^{1}$.

The classes in the Grothendieck ring are then given by $\left[Z_{\alpha}\right]=\left[\mathbb{P}^{1} \times \mathbb{P}^{1}\right]=(\mathbb{L}+1)^{2}=$ $\mathbb{L}^{2}+2 \mathbb{L}+1$, so that $\left[\mathbb{P}^{3} \backslash Z_{\alpha}\right]=\mathbb{L}^{3}+\mathbb{L}^{2}+\mathbb{L}+1-\left(\mathbb{L}^{2}+2 \mathbb{L}+1\right)=\mathbb{L}^{3}-\mathbb{L}$. We then use Lemma 7.1 to compute the class $\left[\mathbb{A}^{5} \backslash \widehat{C Z}_{\alpha}\right]$. We have

$\left[\mathbb{A}^{5} \backslash \widehat{C Z}_{\alpha}\right]=\mathbb{L}^{5}-\mathbb{L}(\mathbb{L}-1)\left[Z_{\alpha}\right]-\mathbb{L}=\mathbb{L}^{5}-\mathbb{L}-\mathbb{L}(\mathbb{L}-1)(\mathbb{L}+1)^{2}=\mathbb{L}^{5}-\mathbb{L}^{4}-\mathbb{L}^{3}+\mathbb{L}^{2}$.

We then use the last identity of Lemma 7.1 to compute

$$
\begin{gathered}
{\left[\mathbb{A}^{5} \backslash\left(\widehat{C Z}_{\alpha} \cup H_{0} \cup H_{1}\right)\right]=\mathbb{L}^{5}-2 \mathbb{L}^{4}-(\mathbb{L}-2)(\mathbb{L}-1)\left[Z_{\alpha}\right]-(\mathbb{L}-2)} \\
=\mathbb{L}^{5}-2 \mathbb{L}^{4}-(\mathbb{L}-2)(\mathbb{L}-1)(\mathbb{L}+1)^{2}-(\mathbb{L}-2)=\mathbb{L}^{5}-3 \mathbb{L}^{4}+\mathbb{L}^{3}+3 \mathbb{L}^{2}-2 \mathbb{L} .
\end{gathered}
$$

Theorem 7.3. Over the quadratic extension $\mathbb{K}=\mathbb{Q}(\sqrt{-1})$, the motive $\mathfrak{m}\left(\mathbb{A}^{5} \backslash\left(\widehat{C Z}_{\alpha} \cup\right.\right.$ $\left.\left.H_{0} \cup H_{1}\right), \Sigma\right)$ is mixed Tate.

Proof. Over $\mathbb{K}=\mathbb{Q}(\sqrt{-1})$, the quadric $Q_{\alpha}$, for $\alpha \in \mathbb{Q}$, satisfies

$$
\left.Q_{\alpha}\right|_{\mathbb{Q}(\sqrt{-1})}=2 \cdot \mathbb{H}
$$

hence the motive is given by (7.3) as

$$
\mathfrak{m}\left(Z_{\alpha}\right)=\mathbb{Z} \oplus \mathbb{Z}(1)[2] \oplus \mathbb{Z}(1)[2] \oplus \mathbb{Z}(2)[4]=\mathfrak{m}\left(\mathbb{P}^{1} \times \mathbb{P}^{1}\right)
$$

where $\mathfrak{m}\left(\mathbb{P}^{1}\right)=\mathbb{Z} \oplus \mathbb{Z}(1)[2]$. This corresponds to the Grothendieck class $\left[Z_{\alpha}\right]=$ $1+2 \mathbb{L}+\mathbb{L}^{2}$. 
The Gysin distinguished triangle of the closed embedding $Z_{\alpha} \hookrightarrow \mathbb{P}^{3}$ of codimension one gives

$$
\mathfrak{m}\left(\mathbb{P}^{3} \backslash Z_{\alpha}\right) \rightarrow \mathfrak{m}\left(\mathbb{P}^{3}\right) \rightarrow \mathfrak{m}\left(Z_{\alpha}\right)(1)[2] \rightarrow \mathfrak{m}\left(\mathbb{P}^{3} \backslash Z_{\alpha}\right)[1]
$$

hence if two of the three terms are in the triangulated subcategory of mixed Tate motives, the third term also is. This implies that $\mathfrak{m}\left(\mathbb{P}^{3} \backslash Z_{\alpha}\right)$ is mixed Tate.

When passing to the projective cone $C Z_{\alpha}$ in $\mathbb{P}^{4}$, since $\mathbb{P}^{4} \backslash C Z_{\alpha} \rightarrow \mathbb{P}^{3} \backslash Z_{\alpha}$ is an $\mathbb{A}^{1}$-fibration, by homotopy invariance we have $\mathfrak{m}_{c}^{j}\left(\mathbb{P}^{4} \backslash C Z_{\alpha}\right)=\mathfrak{m}_{c}^{j-2}\left(\mathbb{P}^{3} \backslash Z_{\alpha}\right)(-1)$, where we consider here the motive $\mathfrak{m}_{c}^{j}$ with compact support that corresponds to the cohomology $H_{c}^{j}$. Thus, if the motive $\mathfrak{m}\left(\mathbb{P}^{3} \backslash Z_{\alpha}\right)$ is mixed Tate, then so is the motive $\mathfrak{m}\left(\mathbb{P}^{4} \backslash C Z_{\alpha}\right)$.

In passing from the motive $\mathfrak{m}\left(\mathbb{P}^{4} \backslash C Z_{\alpha}\right)$ to the motive $\mathfrak{m}\left(\mathbb{A}^{5} \backslash \widehat{C Z}_{\alpha}\right)$, consider the $\mathbb{P}^{1}$-bundle $\mathcal{P}$ compactification of the $\mathbb{G}_{m}$-bundle $\mathcal{T}=\mathbb{A}^{5} \backslash \widehat{C Z}_{\alpha} \rightarrow X=\mathbb{P}^{4} \backslash C Z_{\alpha}$ and the Gysin distinguished triangle

$$
\mathfrak{m}(\mathcal{T}) \rightarrow \mathfrak{m}(\mathcal{P}) \rightarrow \mathfrak{m}_{c}(\mathcal{P} \backslash \mathcal{T})^{*}(1)[2] \rightarrow \mathfrak{m}(\mathcal{T})[1]
$$

see [20], p.197. The motive of a projective bundle satisfies $\mathfrak{m}(\mathcal{P})$ hence $\mathfrak{m}(\mathcal{P})$ is mixed Tate, since $\mathfrak{m}(X)$ is. The motive $\mathfrak{m}_{c}(\mathcal{P} \backslash \mathcal{T})$ is also mixed Tate since $\mathcal{P} \backslash \mathcal{T}$ consists of two copies of $X$, hence the remaining term $\mathfrak{m}(\mathcal{T})$ is also mixed Tate.

We then consider the union of $\widehat{C Z}_{\alpha}$ and the affine hyperplanes $H_{0}=\left\{u_{0}=0\right\}$ and $H_{1}=\left\{u_{0}=1\right\}$ in the affine space $\mathbb{A}^{5}$. In order to check that the motive of the union $\widehat{C Z}_{\alpha} \cup H_{0} \cup H_{1}$ is mixed Tate suffices to know that the motives $\mathfrak{m}\left(\mathbb{A}^{5} \backslash\left(H_{0} \cup H_{1}\right)\right)$ and $\mathfrak{m}\left(\mathbb{A}^{5} \backslash \widehat{C Z}_{\alpha}\right)$ as well as the motive of the intersection $\mathfrak{m}\left(\widehat{C Z}_{\alpha} \cap\left(H_{0} \cup H_{1}\right)\right)$ are mixed Tate. This follows by applying the Mayer-Vietoris distinguished triangle

$$
\mathfrak{m}(U \cap V) \rightarrow \mathfrak{m}(U) \oplus \mathfrak{m}(V) \rightarrow \mathfrak{m}(U \cup V) \rightarrow \mathfrak{m}(U \cap V)[1]
$$

with $U=\mathbb{A}^{5} \backslash \widehat{C Z}_{\alpha}$ and $V=\mathbb{A}^{5} \backslash\left(H_{0} \cup H_{1}\right)$. This shows that it suffices to know two of the three terms are mixed Tate to know the remaining one also is. The motive $\mathfrak{m}\left(\mathbb{A}^{5} \backslash \widehat{C Z}_{\alpha}\right)$ is mixed Tate by our previous argument. The motive $\mathfrak{m}\left(\mathbb{A}^{5} \backslash\left(H_{0} \cup H_{1}\right)\right)$ is also mixed Tate by a similar argument, since $\mathfrak{m}\left(H_{0} \cup H_{1}\right)$ clearly is. Thus, it suffices to show that the motive $\mathfrak{m}\left(\mathbb{A}^{5} \backslash\left(\widehat{C Z}_{\alpha} \cap\left(H_{0} \cup H_{1}\right)\right)\right.$ is mixed Tate, which can be shown by showing that the motive $\mathfrak{m}\left(\widehat{C Z}_{\alpha} \cap\left(H_{0} \cup H_{1}\right)\right)$ is mixed Tate. The intersection $\widehat{C Z}_{\alpha} \cap\left(H_{0} \cup H_{1}\right)$ consists of two sections of the cone, hence one has two copies of the motive $\mathfrak{m}\left(\hat{Z}_{\alpha}\right)$ that is also a Tate motive.

The divisor $\Sigma$ in $\mathbb{A}^{5}$ is a union of coordinate hyperplanes and their translates, and is also mixed Tate. Thus, the motive $\mathfrak{m}\left(\mathbb{A}^{5} \backslash\left(\widehat{C Z}_{\alpha} \cap\left(H_{0} \cup H_{1}\right), \Sigma\right)\right.$ sits in a distinguished triangle in the Voevodsky triangulated category of mixed motives over $\mathbb{Q}$, where two of the three terms, $\mathfrak{m}\left(\mathbb{A}^{5} \backslash\left(\widehat{C Z}_{\alpha} \cap\left(H_{0} \cup H_{1}\right)\right)\right.$ and $\mathfrak{m}(\Sigma)$, are both mixed Tate. This implies that the remaining term $\mathfrak{m}\left(\mathbb{A}^{5} \backslash\left(\widehat{C Z}_{\alpha} \cap\left(H_{0} \cup H_{1}\right), \Sigma\right)\right.$ is also mixed Tate. 
7.5. Pencils of quadrics in $\mathbb{P}^{5}$. We consider then the next step, namely the quadric $Z_{\alpha, 4} \subset \mathbb{P}^{5}$ that occurs in the computation of the $a_{4}$ term of the heat kernel expansion.

Theorem 7.4. Over the quadratic field extension $\mathbb{K}=\mathbb{Q}(\sqrt{-1})$ the quadric $Z_{\alpha, 4} \subset \mathbb{P}^{5}$ determined by the quadratic form

$$
Q_{\alpha, 4}=u_{1}^{2}+\frac{1}{\alpha^{2}}\left(u_{2}^{2}+u_{3}^{2}+u_{4}^{2}\right)+u_{5}^{2}+u_{6}^{2}
$$

has Grothendieck class $\left[\mathbb{P}^{5} \backslash Z_{\alpha, 4}\right]=\mathbb{L}^{5}-\mathbb{L}^{2}$. The class of the complement in $\mathbb{A}^{7}$ of $\widehat{C Z}_{\alpha, 4}$ has Grothendieck class

$$
\left[\mathbb{A}^{7} \backslash \widehat{C Z}_{\alpha, 4}\right]=\mathbb{L}^{7}-\mathbb{L}^{6}-\mathbb{L}^{4}+\mathbb{L}^{3}
$$

and the class of the complement of the union $\widehat{C Z}_{\alpha, 4} \cup H_{0} \cup H_{1}$ is given by

$$
\left[\mathbb{A}^{7} \backslash\left(\widehat{C Z}_{\alpha, 4} \cup H_{0} \cup H_{1}\right)\right]=\mathbb{L}^{7}-3 \mathbb{L}^{6}+2 \mathbb{L}^{5}-\mathbb{L}^{4}+3 \mathbb{L}^{3}-2 \mathbb{L}^{2} .
$$

Proof. Over $\mathbb{K}=\mathbb{Q}(\sqrt{-1})$ we can consider the change of coordinates

$$
X=u_{5}+i u_{6}, \quad Y=u_{5}-i u_{6} .
$$

With this change of coordinates, we rewrite the quadratic form as

$$
Q_{\alpha, 4}=Q_{\alpha, 2}+X Y
$$

where $Q_{\alpha, 2}=u_{1}^{2}+\frac{1}{\alpha^{2}}\left(u_{2}^{2}+u_{3}^{2}+u_{4}^{2}\right)$ is the quadratic form of the $a_{2}$-term that we discussed in 97.4 . We can compute the Grothendieck class $\left[\hat{Z}_{\alpha, 4}\right]$ by considering the two possible cases $Y \neq 0$ and $Y=0$. In the first case, since $Y \neq 0$ we have solutions of the form

$$
X=-\frac{Q_{\alpha, 2}\left(u_{1}, u_{2}, u_{3}, u_{4}\right)}{Y},
$$

which give a choice of $Y \in \mathbb{G}_{m}$ and arbitrary $\left(u_{1}, u_{2}, u_{3}, u_{4}\right) \in \mathbb{A}^{4}$, hence a contribution of $(\mathbb{L}-1) \mathbb{L}^{4}$ to the class. In the case where $Y=0$, we have solutions given by an arbitrary $X \in \mathbb{A}^{1}$ and $\left(u_{1}, u_{2}, u_{3}, u_{4}\right) \in \hat{Z}_{\alpha, 1}$. This gives a contribution of $\mathbb{L}\left[\hat{Z}_{\alpha, 2}\right]$ to the class. Thus, we obtain

$$
\left[\hat{Z}_{\alpha, 4}\right]=(\mathbb{L}-1) \mathbb{L}^{4}+\mathbb{L}\left[\hat{Z}_{\alpha, 1}\right]=(\mathbb{L}-1) \mathbb{L}^{4}+\mathbb{L}(\mathbb{L}-1)(\mathbb{L}+1)^{2}+\mathbb{L},
$$

since $\left[\hat{Z}_{\alpha, 2}\right]=(\mathbb{L}-1)\left[Z_{\alpha, 1}\right]+1$ and $\left[Z_{\alpha, 1}\right]=\left[\mathbb{P}^{1} \times \mathbb{P}^{1}\right]$. This gives

$$
\left[\mathbb{A}^{6} \backslash \hat{Z}_{\alpha, 4}\right]=\mathbb{L}^{6}-\mathbb{L}-(\mathbb{L}-1)\left(\mathbb{L}^{4}+\mathbb{L}^{3}+2 \mathbb{L}^{2}+\mathbb{L}\right)=(\mathbb{L}-1)\left(\mathbb{L}^{5}-\mathbb{L}^{2}\right) .
$$

Since $\left[\mathbb{A}^{6} \backslash \hat{Z}_{\alpha, 4}\right]=(\mathbb{L}-1)\left[\mathbb{P}^{5} \backslash Z_{\alpha, 2}\right]$ we then have $\left[\mathbb{P}^{5} \backslash Z_{\alpha, 4}\right]=\mathbb{L}^{5}-\mathbb{L}^{2}$, as stated. Thus, we also have $\left[Z_{\alpha, 4}\right]=\left[\mathbb{P}^{5}\right]-\left(\mathbb{L}^{5}-\mathbb{L}^{2}\right)=\mathbb{L}^{4}+\mathbb{L}^{3}+2 \mathbb{L}^{2}+\mathbb{L}+1$. Using Lemma 7.1 we then obtain

$$
\begin{gathered}
{\left[\mathbb{A}^{7} \backslash \widehat{C Z}_{\alpha, 4}\right]=\mathbb{L}^{7}-\mathbb{L}(\mathbb{L}-1)\left[Z_{\alpha, 4}\right]-\mathbb{L}} \\
=\mathbb{L}^{7}-\mathbb{L}(\mathbb{L}-1)\left(\mathbb{L}^{4}+\mathbb{L}^{3}+2 \mathbb{L}^{2}+\mathbb{L}+1\right)-\mathbb{L}=\mathbb{L}^{7}-\mathbb{L}^{6}-\mathbb{L}^{4}+\mathbb{L}^{3} .
\end{gathered}
$$

The Grothendieck class of the complement of the union of $\widehat{C Z}_{\alpha, 4}$ and the two hyperplanes is given by

$$
\left[\mathbb{A}^{7} \backslash\left(\widehat{C Z}_{\alpha, 4} \cup H_{0} \cup H_{1}\right)\right]=\mathbb{L}^{7}-2 \mathbb{L}^{6}-(\mathbb{L}-1)(\mathbb{L}-2)\left[Z_{\alpha, 4}\right]-(\mathbb{L}-2)
$$


$=\mathbb{L}^{7}-2 \mathbb{L}^{6}-(\mathbb{L}-1)(\mathbb{L}-2)\left(\mathbb{L}^{4}+\mathbb{L}^{3}+2 \mathbb{L}^{2}+\mathbb{L}+1\right)-(\mathbb{L}-2)=\mathbb{L}^{7}-3 \mathbb{L}^{6}+2 \mathbb{L}^{5}-\mathbb{L}^{4}+3 \mathbb{L}^{3}-2 \mathbb{L}^{2}$.

We also have the analog of Theorem 7.3 , which we state here. The proof is analogous and we omit it.

Proposition 7.5. Over the quadratic extension $\mathbb{K}=\mathbb{Q}(\sqrt{-1})$, the motive $\mathfrak{m}\left(\mathbb{A}^{7} \backslash\right.$ $\left.\left(\widehat{C Z}_{\alpha, 4} \cup H_{0} \cup H_{1}\right), \Sigma\right)$ is mixed Tate.

Proof. The argument is completely analogous to Theorem 7.3 , using the fact that, over $\mathbb{K}=\mathbb{Q}(\sqrt{-1})$ the quadratic form is

$$
\left.Q_{\alpha, 4}\right|_{\mathbb{Q}(\sqrt{-1})}=3 \cdot \mathbb{H} .
$$

The rest of the argument follows as in Theorem 7.3 .

7.6. The Grothendieck class of $\mathbb{P}^{2 n-1} \backslash Z_{\alpha, 2 n}$ over $\mathbb{K}=\mathbb{Q}(\sqrt{-1})$. The argument of Theorem 7.4 can be used to obtain an inductive argument computing the Grothendieck class $\left[\mathbb{P}^{2 n-1} \backslash Z_{\alpha, 2 n}\right]$ for all the quadrics $Z_{\alpha, n}$ determined by the quadratic forms

$$
Q_{\alpha, 2 n}=u_{1}^{2}+\frac{1}{\alpha^{2}}\left(u_{2}^{2}+u_{3}^{2}+u_{4}^{2}\right)+u_{5}^{2}+u_{6}^{2}+\cdots+u_{2 n+1}^{2}+u_{2 n+2}^{2},
$$

for all $n \geq 3$.

Theorem 7.6. Over the quadratic field extension $\mathbb{K}=\mathbb{Q}(\sqrt{-1})$ the quadric $Z_{\alpha, 2 n}$ has Grothendieck class $\left[\mathbb{P}^{2 n+1} \backslash Z_{\alpha, 2 n}\right]=\mathbb{L}^{2 n+1}-\mathbb{L}^{n}$. The affine complement of $\widehat{C Z}_{\alpha, 2 n}$ has class

$$
\left[\mathbb{A}^{2 n+3} \backslash \widehat{C Z}_{\alpha, 2 n}\right]=\mathbb{L}^{2 n+3}-\mathbb{L}^{2 n+2}-\mathbb{L}^{n+2}+\mathbb{L}^{n+1}
$$

and the affine complement of the union $\widehat{C Z}_{\alpha, 2 n} \cup H_{0} \cup H_{1}$ has class

$$
\left[\mathbb{A}^{2 n+3} \backslash\left(\widehat{C Z}_{\alpha, 2 n} \cup H_{0} \cup H_{1}\right)\right]=\mathbb{L}^{2 n+3}-3 \mathbb{L}^{2 n+2}+2 \mathbb{L}^{2 n+1}-\mathbb{L}^{n+2}+3 \mathbb{L}^{n+1}-2 \mathbb{L}^{n} .
$$

Proof. We proceed as in Theorem [.4. Over the field $\mathbb{K}=\mathbb{Q}(\sqrt{-1})$ the change of coordinates

$$
X=u_{2 n+1}+i u_{2 n+2}, \quad Y=u_{2 n+1}-i u_{2 n+2}
$$

puts $Q_{\alpha, 2 n}$ in the form

$$
Q_{\alpha, 2 n}=Q_{\alpha, 2 n-2}\left(u_{1}, \ldots, u_{2 n}\right)+X Y .
$$

Thus, the Grothendieck class $\left[\hat{Z}_{\alpha, 2 n}\right]$ is a sum of a contribution corresponding to $Y \neq 0$, which is of the form $(\mathbb{L}-1) \mathbb{L}^{2 n}$ and a contribution from $Y=0$, which is of the form $\mathbb{L}\left[\hat{Z}_{\alpha, n-1}\right]$. This gives

$$
\left[\mathbb{A}^{2 n+2} \backslash \hat{Z}_{\alpha, 2 n}\right]=\mathbb{L}^{2 n+2}-2 \mathbb{L}^{2 n+1}+\mathbb{L}^{2 n}+\mathbb{L}\left[\mathbb{A}^{2 n} \backslash \hat{Z}_{\alpha, 2 n-2}\right],
$$

hence using the relation between the classes of the affine and projective complements,

$$
\left[\mathbb{P}^{2 n+1} \backslash Z_{\alpha, 2 n}\right]=\mathbb{L}^{2 n}(\mathbb{L}-1)+\mathbb{L}\left[\mathbb{P}^{2 n-1} \backslash Z_{\alpha, 2 n-2}\right] .
$$


Assuming inductively that $\left[\mathbb{P}^{2 n-1} \backslash Z_{\alpha, 2 n-2}\right]=\mathbb{L}^{2 n-1}-\mathbb{L}^{n-1}$ we indeed obtain that the class of the complement is $\left[\mathbb{P}^{2 n+1} \backslash Z_{\alpha, 2 n}\right]=\mathbb{L}^{2 n}(\mathbb{L}-1)+\mathbb{L}\left(\mathbb{L}^{2 n-1}-\mathbb{L}^{n-1}\right)=\mathbb{L}^{2 n+1}-\mathbb{L}^{n}$. We then have

$\left[Z_{\alpha, 2 n}\right]=\left[\mathbb{P}^{2 n+1}\right]-\left[\mathbb{P}^{2 n+1} \backslash Z_{\alpha, 2 n}\right]=\mathbb{L}^{2 n}+\mathbb{L}^{2 n-1}+\cdots+\mathbb{L}^{n+1}+2 \mathbb{L}^{n}+\mathbb{L}^{n-1}+\cdots+\mathbb{L}^{2}+\mathbb{L}+1$.

Using Lemma 7.1, we obtain

$$
\begin{gathered}
{\left[\mathbb{A}^{2 n+3} \backslash \widehat{C Z}_{\alpha, 2 n}\right]=\mathbb{L}^{2 n-3}-\mathbb{L}(\mathbb{L}-1)\left[Z_{\alpha, 2 n}\right]-\mathbb{L}} \\
=\mathbb{L}^{2 n+3}-\sum_{j=2}^{n} \mathbb{L}^{j}-\mathbb{L}^{n+1}-2 \mathbb{L}^{n+2}-\sum_{j=n+3}^{2 n+1} \mathbb{L}^{j}-\mathbb{L}^{2 n+2}+\sum_{j=2}^{n} \mathbb{L}^{j}+2 \mathbb{L}^{n+1}+\mathbb{L}^{n+2}+\sum_{j=n+3}^{2 n+1} \mathbb{L}^{j} \\
=\mathbb{L}^{2 n+3}+\mathbb{L}^{n+1}-\mathbb{L}^{n+2}-\mathbb{L}^{2 n+2} .
\end{gathered}
$$

We proceed in the same way for the computation of the class of the affine complement of the union $\widehat{C Z}_{\alpha, 2 n} \cup H_{0} \cup H_{1}$, using Lemma 7.1. We have

$$
\left[\mathbb{A}^{2 n+3} \backslash\left(\widehat{C Z}_{\alpha, 2 n} \cup H_{0} \cup H_{1}\right)\right]=\mathbb{L}^{2 n+3}-2 \mathbb{L}^{2 n+2}-(\mathbb{L}-2)(\mathbb{L}-1)\left[Z_{\alpha, 2 n}\right]-(\mathbb{L}-2)
$$

and using again the expression

$$
\left[Z_{\alpha, 2 n}\right]=\mathbb{L}^{2 n}+\mathbb{L}^{2 n-1}+\cdots+\mathbb{L}^{n+1}+2 \mathbb{L}^{n}+\mathbb{L}^{n-1}+\cdots+\mathbb{L}^{2}+\mathbb{L}+1
$$

we obtain

$$
(\mathbb{L}-2)(\mathbb{L}-1)\left[Z_{\alpha, 2 n}\right]=2-\mathbb{L}+2 \mathbb{L}^{n}-3 \mathbb{L}^{n+1}+\mathbb{L}^{n+2}-2 \mathbb{L}^{n+1}+\mathbb{L}^{2 n+2}
$$

due to cancellations of terms similar to the previous case. We then have

$$
\begin{gathered}
\mathbb{L}^{2 n+3}-2 \mathbb{L}^{2 n+2}-(\mathbb{L}-2)(\mathbb{L}-1)\left[Z_{\alpha, 2 n}\right]-(\mathbb{L}-2)= \\
\mathbb{L}^{2 n+3}-3 \mathbb{L}^{2 n+2}+2 \mathbb{L}^{2 n+1}-\mathbb{L}^{n+2}+3 \mathbb{L}^{n+1}-2 \mathbb{L}^{n},
\end{gathered}
$$

which agrees with the cases $n=1$ and $n=2$ computed in Theorems 7.2 and 7.4 .

We also have the analog of Theorem 7.3 and Proposition 7.5 , proved by the same argument.

Proposition 7.7. Over the field extension $\mathbb{K}=\mathbb{Q}(\sqrt{-1})$, the mixed motive

$$
\mathfrak{m}\left(\mathbb{A}^{2 n+3} \backslash\left(\widehat{C Z}_{\alpha, 2 n} \cup H_{0} \cup H_{1}\right), \Sigma\right)
$$

is mixed Tate.

Proof. The argument is completely analogous to Theorem 7.3 , using the fact that, over $\mathbb{K}=\mathbb{Q}(\sqrt{-1})$ the quadratic form is

$$
\left.Q_{\alpha, 2 n}\right|_{\mathbb{Q}(\sqrt{-1})}=(n+1) \cdot \mathbb{H},
$$

with (17.3) giving the motive $\mathfrak{m}\left(\left.Q_{\alpha, 2 n}\right|_{\mathbb{Q}(\sqrt{-1})}\right)$. The motives of complements, and projective and affine cones and the relative motives $\mathfrak{m}\left(\mathbb{A}^{2 n+3} \backslash \widehat{C Z}_{\alpha, 2 n}, \Sigma\right)$ and $\mathfrak{m}\left(\mathbb{A}^{2 n+3} \backslash\right.$ $\left.\left(\widehat{C Z}_{\alpha, 2 n} \cup H_{0} \cup H_{1}\right), \Sigma\right)$ are then obtained as in Theorem 7.3 , 
7.7. The motive of $Z_{\alpha, 2 n}$ over $\mathbb{Q}$. Over the rationals, the quadratic form $Q_{\alpha, 2 n}$ is anisotropic, although, as we have seen, it becomes isotropic over the field extension $\mathbb{K}=\mathbb{Q}(\sqrt{-1})$, with $\left.Q_{\alpha, 2 n}\right|_{\mathbb{Q}(\sqrt{-1})}=(n+1) \cdot \mathbb{H}$. The motive of $Z_{\alpha, 2 n}$ over $\mathbb{Q}(\sqrt{-1})$ is a sum of Tate motives

$$
\mathfrak{m}\left(\left.Z_{\alpha, 2 n}\right|_{\mathbb{K}}\right)=\mathbb{Z}(n)[2 n] \oplus \mathbb{Z}(n)[2 n] \oplus \bigoplus_{i=0, \ldots, n-1, n+1, \ldots 2 n} \mathbb{Z}(i)[2 i],
$$

which corresponds to the Grothendieck class $\left[Z_{\alpha, 2 n}\right]=\left[\mathbb{P}^{2 n+1}\right]-\left[\mathbb{P}^{2 n+1} \backslash Z_{\alpha, 2 n}\right]=$ $1+\cdots+\mathbb{L}^{2 n+1}-\left(\mathbb{L}^{2 n+1}-\mathbb{L}^{n}\right)=1+\mathbb{L}+\cdots+\mathbb{L}^{n-1}+2 \mathbb{L}^{n}+\mathbb{L}^{n+1}+\cdots+\mathbb{L}^{2 n}$. Over the field $\mathbb{Q}$, the motive of $Z_{\alpha, 2 n}$ is given by (7.5), with

$$
\mathfrak{m}\left(\left.Z_{\alpha, 2 n}\right|_{\mathbb{Q}}\right)=\mathfrak{m}_{1} \oplus \mathfrak{m}_{1}(1)[2]
$$

when $n$ is odd and

$$
\mathfrak{m}\left(\left.Z_{\alpha, 2 n}\right|_{\mathbb{Q}}\right)=\mathfrak{m}_{1} \oplus \mathcal{R}_{Q, \mathbb{Q}, n} \oplus \mathfrak{m}_{1}(1)[2]
$$

when $n$ is even, where $\mathcal{R}_{Q, \mathbb{Q}, n}$ is a form of a Tate motive denoted by $\mathcal{R}_{Q, \mathbb{Q}, n}=$ $\mathbb{Q}\left(\sqrt{\operatorname{det}\left(Q_{\alpha, 2 n}\right)}\right)(n)[2 n]$ in [19]. When passing to the quadratic field extension $\mathbb{Q}(\sqrt{-1})$ these motivic decompositions become the decomposition into Tate motives given above.

\section{Appendix A: Full expression fOR $b_{-6}$}

Here we provide the full expression of the term $b_{-6}$ given by (5.4), which we used for the calculation of the term $a_{4}$ :

$$
\begin{aligned}
& b_{-6}\left(t, \eta, \xi_{1}, \ldots, \xi_{6}\right)= \\
& \frac{2560 \xi_{3}^{8} a^{\prime}(t)^{4} \csc ^{8}(\eta)}{\alpha^{12} Q^{7}}-\frac{53760 \xi_{1}^{2} \xi_{3}^{8} a^{\prime}(t)^{4} \csc ^{8}(\eta)}{\alpha^{12} Q^{8}}+\frac{107520 \xi_{1}^{4} \xi_{3}^{8} a^{\prime}(t)^{4} \csc ^{8}(\eta)}{\alpha^{12} Q^{9}}-\frac{640 \xi_{3}^{6} a^{\prime}(t)^{2} \csc ^{8}(\eta)}{\alpha^{10} Q^{6}}+ \\
& \frac{5120 \cot ^{2}(\eta) \xi_{3}^{8} a^{\prime}(t)^{2} \csc ^{8}(\eta)}{1^{12} Q^{7}}+\frac{5760 \xi_{1}^{2} \xi_{3}^{6} a^{\prime}(t)^{2} \csc ^{8}(\eta)}{\alpha^{10} Q^{7}}+\frac{5120 \xi_{2}^{2} \xi_{3}^{6} a^{\prime}(t)^{2} \csc ^{8}(\eta)}{\alpha^{12} Q^{7} \operatorname{los}_{4}}+\frac{10240 \xi_{3}^{6} \xi_{4}^{2} a^{\prime}(t)^{2} \csc ^{8}(\eta)}{12 Q_{3}^{7}}- \\
& \frac{53760 \cot ^{2}(\eta) \xi_{1}^{2} \xi_{3}^{8} a^{\prime}(t)^{2} \csc ^{8}(\eta)}{\alpha^{12} Q_{\alpha}^{8}}-\frac{53760 \cot ^{2}(\eta) \xi_{2}^{2} \xi_{3}^{8} a^{\prime}(t)^{2} \csc ^{8}(\eta)}{{ }^{14} Q^{8}}-\frac{53760 \xi_{1}^{2} \xi_{2}^{2} \xi_{3}^{6} a^{\prime}(t)^{2} \csc ^{8}(\eta)}{12}-\frac{107520 \xi_{1}^{2} \xi_{3}^{6} \xi_{4}^{2} a^{\prime}(t)^{2} \csc ^{8}(\eta)}{\alpha^{12} Q^{8}}-
\end{aligned}
$$

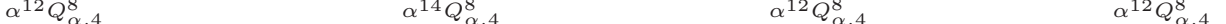

$$
\begin{aligned}
& \frac{107520 \xi_{2}^{2} \xi_{3}^{6} \xi_{4}^{2} a^{\prime}(t)^{2} \csc ^{8}(\eta)}{\alpha^{14} Q^{8}, 4}+\frac{645120 \cot ^{2}(\eta) \xi_{1}^{2} \xi_{2}^{2} \xi_{3}^{8} a^{\prime}(t)^{2} \csc ^{8}(\eta)}{\alpha^{14} Q_{\alpha, 4}^{9}}+\frac{1290240 \xi_{1}^{2} \xi_{2}^{2} \xi_{3}^{6} \xi_{4}^{2} a^{\prime}(t)^{2} \csc ^{8}(\eta)}{\alpha^{14} Q_{\alpha, 4}^{9}}+\frac{112 \xi_{3}^{4} \csc ^{8}(\eta)}{\alpha^{8} Q_{\alpha, 4}^{5}}- \\
& \frac{1664 \cot ^{2}(\eta) \xi_{3}^{6} \csc ^{8}(\eta)}{\alpha^{10} Q^{6}}-\frac{1664 \xi_{2}^{2} \xi_{3}^{4} \csc ^{8}(\eta)}{\alpha^{10} Q^{6}}+\frac{2560 \cot ^{4}(\eta) \xi_{3}^{8} \csc ^{8}(\eta)}{\alpha^{12} Q^{7}}+\frac{30080 \cot ^{2}(\eta) \xi_{2}^{2} \xi_{3}^{6} \csc ^{8}(\eta)}{\alpha^{12} Q^{7}}+\frac{2560 \xi_{2}^{4} \xi_{3}^{4} \csc ^{8}(\eta)}{\alpha^{12} Q^{7}}- \\
& \frac{10240 \xi_{3}^{6} \xi_{4}^{2} \csc ^{8}(\eta)}{\alpha^{12} Q_{\alpha, 4}^{7}}-\frac{53760 \cot ^{4}(\eta) \xi_{2}^{2} \xi_{3}^{8} \csc ^{8}(\eta)}{\alpha^{14} Q_{\alpha, 4}^{8}}-\frac{53760 \cot ^{2}(\eta) \xi_{2}^{4} \xi_{3}^{6} \csc ^{8}(\eta)}{\alpha^{14} Q_{\alpha, 4}^{8}}+\frac{215040 \xi_{2}^{2} \xi_{3}^{6} \xi_{4}^{2} \csc ^{8}(\eta)}{\alpha^{14} Q_{\alpha, 4}^{8}}+ \\
& \frac{107520 \cot ^{4}(\eta) \xi_{2}^{4} \xi_{3}^{8} \csc ^{8}(\eta)}{\alpha^{16} Q_{\alpha}^{9}}-\frac{430080 \xi_{2}^{4} \xi_{3}^{6} \xi_{4}^{2} \csc ^{8}(\eta)}{\alpha^{16} Q_{\alpha}^{9}}-\frac{2752 \xi_{3}^{6} a^{\prime}(t)^{4} \csc ^{6}(\eta)}{\alpha^{10} Q_{\alpha, 4}^{6}}+\frac{47040 \xi_{1}^{2} \xi_{3}^{6} a^{\prime}(t)^{4} \csc ^{6}(\eta)}{\alpha^{10} Q_{\alpha, 4}^{7}}+\frac{10240 \xi_{2}^{2} \xi_{3}^{6} a^{\prime}(t)^{4} \csc ^{6}(\eta)}{\alpha^{12} Q_{\alpha, 4}^{7}}+ \\
& \frac{10240 \sec ^{2}(\eta) \xi_{3}^{6} \xi_{4}^{2} a^{\prime}(t)^{4} \csc ^{6}(\eta)}{\alpha^{12} Q_{\alpha, 4}^{7}}-\frac{80640 \xi_{1}^{4} \xi_{3}^{6} a^{\prime}(t)^{4} \csc ^{6}(\eta)}{\alpha^{10} Q_{\alpha, 4}^{8}}-\frac{215040 \xi_{1}^{2} \xi_{2}^{2} \xi_{3}^{6} a^{\prime}(t)^{4} \csc ^{6}(\eta)}{\alpha^{12} Q_{\alpha, 4}^{8}}-\frac{215040 \sec ^{2}(\eta) \xi_{1}^{2} \xi_{3}^{6} \xi_{4}^{2} a^{\prime}(t)^{4} \csc ^{6}(\eta)}{\alpha^{12} Q_{\alpha, 4}^{8}}+ \\
& \frac{430080 \xi_{1}^{4} \xi_{2}^{2} \xi_{3}^{6} a^{\prime}(t)^{4} \csc ^{6}(\eta)}{\alpha^{12} Q_{\alpha, 4}^{9}}+\frac{430080 \sec ^{2}(\eta) \xi_{1}^{4} \xi_{3}^{6} \xi_{4}^{2} a^{\prime}(t)^{4} \csc ^{6}(\eta)}{\alpha^{12} Q_{\alpha, 4}^{9}}+\frac{432 \xi_{3}^{4} a^{\prime}(t)^{2} \csc ^{6}(\eta)}{\alpha^{8} Q_{\alpha, 4}^{5}}-\frac{5760 \cot ^{2}(\eta) \xi_{3}^{6} a^{\prime}(t)^{2} \csc ^{6}(\eta)}{\alpha^{10} Q_{\alpha, 4}^{6}}+ \\
& \frac{1280 \cot (\eta) \cot (2 \eta) \xi_{3}^{6} a^{\prime}(t)^{2} \csc ^{6}(\eta)}{\alpha^{10} Q_{\alpha, 4}^{6}}-\frac{2640 \xi_{1}^{2} \xi_{3}^{4} a^{\prime}(t)^{2} \csc ^{6}(\eta)}{\alpha^{8} Q_{\alpha, 4}^{6}}-\frac{6080 \xi_{2}^{2} \xi_{3}^{4} a^{\prime}(t)^{2} \csc ^{6}(\eta)}{\alpha^{10} Q_{\alpha, 4}^{6}}-\frac{1280 \sec ^{2}(\eta) \xi_{3}^{4} \xi_{4}^{2} a^{\prime}(t)^{2} \csc ^{6}(\eta)}{\alpha^{10} Q_{\alpha, 4}^{6}}- \\
& \frac{6720 \xi_{3}^{4} \xi_{4}^{2} a^{\prime}(t)^{2} \csc ^{6}(\eta)}{\alpha^{10} Q_{\alpha, 4}^{6}}+\frac{47040 \cot ^{2}(\eta) \xi_{1}^{2} \xi_{3}^{6} a^{\prime}(t)^{2} \csc ^{6}(\eta)}{\alpha^{10} Q_{\alpha, 4}^{7}}-\frac{11520 \cot (\eta) \cot (2 \eta) \xi_{1}^{2} \xi_{3}^{6} a^{\prime}(t)^{2} \csc ^{6}(\eta)}{\alpha^{10} Q_{\alpha, 4}^{7}}+ \\
& \frac{79040 \cot ^{2}(\eta) \xi_{2}^{2} \xi_{3}^{6} a^{\prime}(t)^{2} \csc ^{6}(\eta)}{\alpha^{12} Q_{\alpha, 4}^{7}}-\frac{15360 \cot (\eta) \cot (2 \eta) \xi_{2}^{2} \xi_{3}^{6} a^{\prime}(t)^{2} \csc ^{6}(\eta)}{\alpha^{12} Q_{\alpha, 4}^{7}}+\frac{10240 \xi_{2}^{4} \xi_{3}^{4} a^{\prime}(t)^{2} \csc ^{6}(\eta)}{\alpha^{12} Q_{\alpha, 4}^{7}}+\frac{49920 \xi_{1}^{2} \xi_{2}^{2} \xi_{3}^{4} a^{\prime}(t)^{2} \csc ^{6}(\eta)}{\alpha^{10} Q_{\alpha, 4}^{7}}+ \\
& \frac{5120 \sec ^{2}(\eta) \xi_{3}^{4} \xi_{4}^{4} a^{\prime}(t)^{2} \csc ^{6}(\eta)}{\alpha^{12} Q_{\alpha, 4}^{7}}-\frac{10240 \sec ^{2}(\eta) \xi_{3}^{6} \xi_{4}^{2} a^{\prime}(t)^{2} \csc ^{6}(\eta)}{\alpha^{12} Q_{\alpha, 4}^{7}}+\frac{11520 \sec ^{2}(\eta) \xi_{1}^{2} \xi_{3}^{4} \xi_{4}^{2} a^{\prime}(t)^{2} \csc ^{6}(\eta)}{\alpha^{10} Q_{\alpha, 4}^{7}}+ \\
& \frac{55680 \xi_{1}^{2} \xi_{3}^{4} \xi_{4}^{2} a^{\prime}(t)^{2} \csc ^{6}(\eta)}{\alpha^{10} Q_{\alpha, 4}^{7}}+\frac{10240 \sec ^{2}(\eta) \xi_{2}^{2} \xi_{3}^{4} \xi_{4}^{2} a^{\prime}(t)^{2} \csc ^{6}(\eta)}{\alpha^{12} Q_{\alpha, 4}^{7}}+\frac{89280 \xi_{2}^{2} \xi_{3}^{4} \xi_{4}^{2} a^{\prime}(t)^{2} \csc ^{6}(\eta)}{\alpha^{12} Q_{\alpha, 4}^{7}}-\frac{107520 \cot ^{2}(\eta) \xi_{2}^{4} \xi_{3}^{6} a^{\prime}(t)^{2} \csc ^{6}(\eta)}{\alpha^{14} Q_{\alpha, 4}^{8}}- \\
& \frac{779520 \cot ^{2}(\eta) \xi_{1}^{2} \xi_{2}^{2} \xi_{3}^{6} a^{\prime}(t)^{2} \csc ^{6}(\eta)}{\alpha^{12} Q_{\alpha, 4}^{8}}+\frac{161280 \cot (\eta) \cot (2 \eta) \xi_{1}^{2} \xi_{2}^{2} \xi_{3}^{6} a^{\prime}(t)^{2} \csc ^{6}(\eta)}{\alpha^{12} Q_{\alpha, 4}^{8}}-\frac{107520 \xi_{1}^{2} \xi_{2}^{4} \xi_{3}^{4} a^{\prime}(t)^{2} \csc ^{6}(\eta)}{\alpha^{12} Q_{\alpha, 4}^{8}}-
\end{aligned}
$$




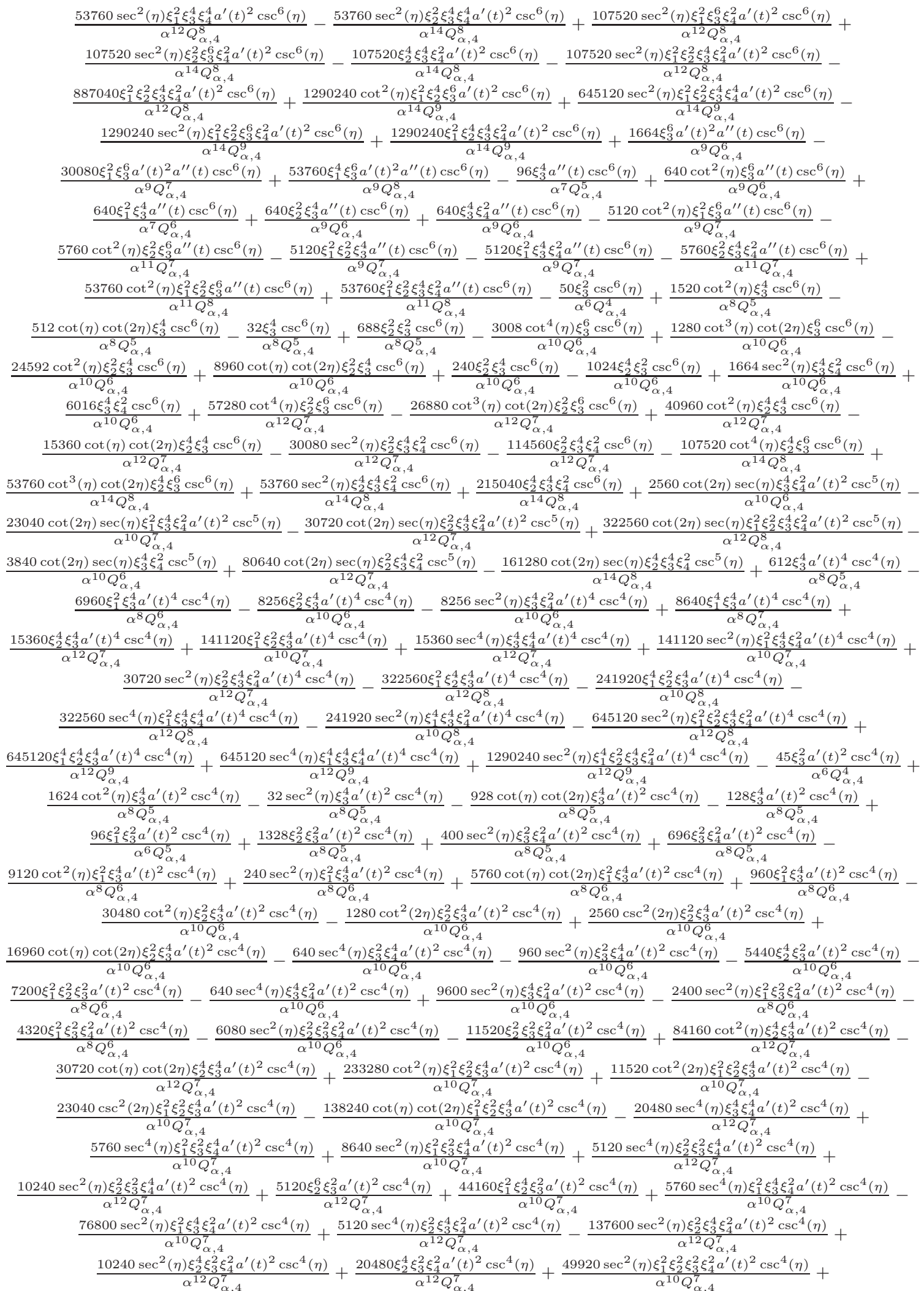




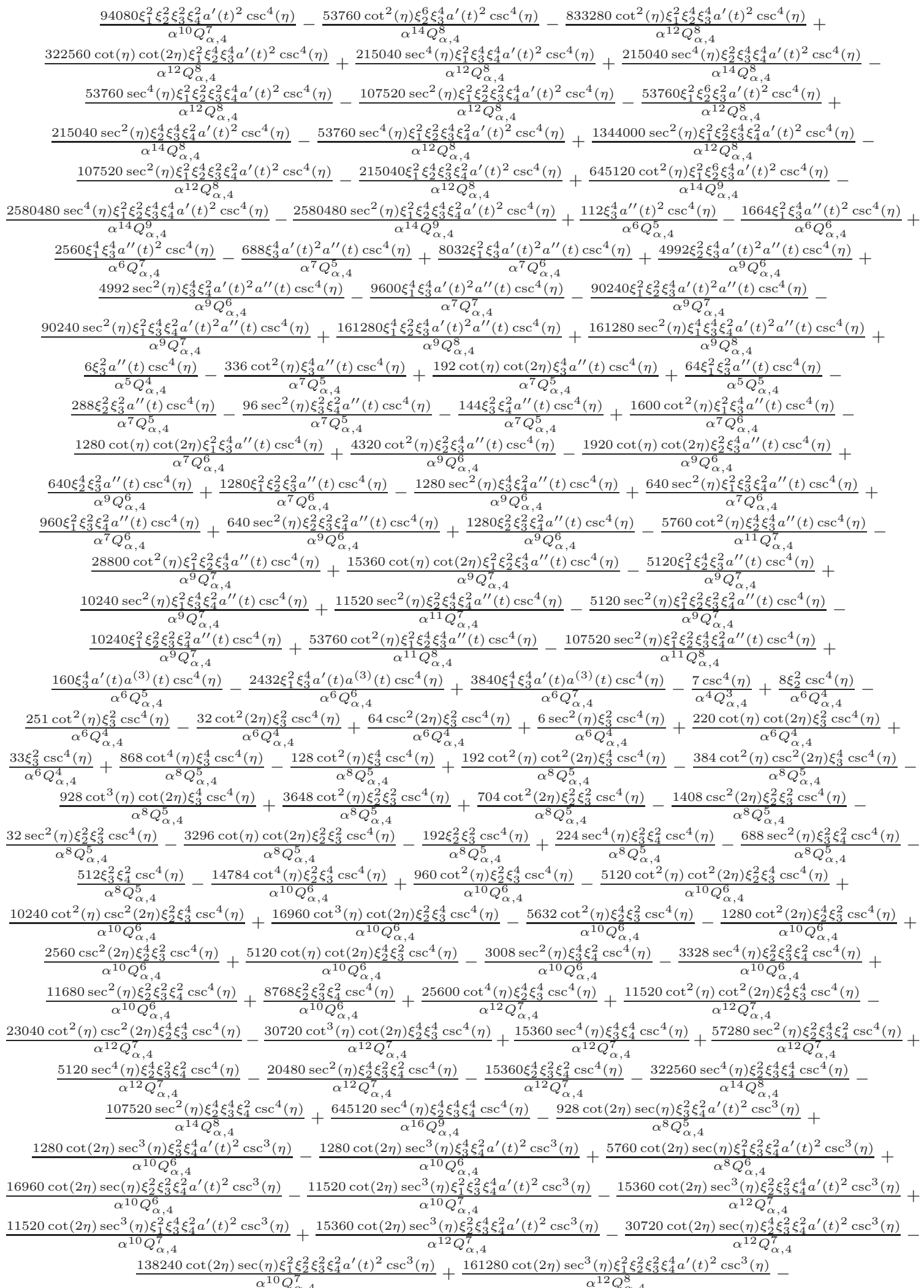


$\frac{161280 \cot (2 \eta) \sec ^{3}(\eta) \xi_{1}^{2} \xi_{2}^{2} \xi_{3}^{4} \xi_{4}^{2} a^{\prime}(t)^{2} \csc ^{3}(\eta)}{\alpha^{12} Q^{8}}+\frac{322560 \cot (2 \eta) \sec (\eta) \xi_{1}^{2} \xi_{2}^{4} \xi_{3}^{2} \xi_{4}^{2} a^{\prime}(t)^{2} \csc ^{3}(\eta)}{\alpha^{12} Q^{8}}+$ $\frac{192 \cot (2 \eta) \sec (\eta) \xi_{3}^{2} \xi_{4}^{2} a^{\prime \prime}(t) \csc ^{3}(\eta)}{\alpha^{7} Q^{5}}-\frac{1280 \cot (2 \eta) \sec (\eta) \xi_{1}^{2} \xi_{3}^{2} \xi_{4}^{2} a^{\prime \prime}(t) \csc ^{3}(\eta)}{\alpha^{7} Q^{6}}-\frac{1920 \cot (2 \eta) \sec (\eta) \xi_{2}^{2} \xi_{3}^{2} \xi_{4}^{2} a^{\prime \prime}(t) \csc ^{3}(\eta)}{\alpha^{9} Q^{6}}+$ $\frac{15360 \cot (2 \eta) \sec (\eta) \xi_{1}^{2} \xi_{2}^{2} \xi_{3}^{2} \xi_{4}^{2} a^{\prime \prime}(t) \csc ^{3}(\eta)}{\alpha^{9} Q^{7}}-\frac{12 \cot (2 \eta) \sec (\eta) \xi_{3}^{2} \csc ^{3}(\eta)}{\alpha^{6} Q^{4}}+\frac{96 \cot (2 \eta) \sec (\eta) \xi_{2}^{2} \xi_{3}^{2} \csc ^{3}(\eta)}{\alpha^{8} Q^{5}}+$ $\frac{928 \cot (2 \eta) \sec (\eta) \xi_{3}^{2} \xi_{4}^{2} \csc ^{3}(\eta)}{\alpha^{8} Q_{\alpha, 4}^{5}}-\frac{16960 \cot (2 \eta) \sec (\eta) \xi_{2}^{2} \xi_{3}^{2} \xi_{4}^{2} \csc ^{3}(\eta)}{\alpha^{10} Q_{\alpha, 4}^{6}}+\frac{30720 \cot (2 \eta) \sec (\eta) \xi_{2}^{4} \xi_{3}^{2} \xi_{4}^{2} \csc ^{3}(\eta)}{\alpha^{12} Q_{\alpha, 4}^{7}}-\frac{7 \xi_{3}^{2} a^{\prime}(t)^{4} \csc ^{2}(\eta)}{\alpha^{6} Q_{\alpha, 4}^{4}}-$ $\frac{216 \xi_{1}^{2} \xi_{3}^{2} a^{\prime}(t)^{4} \csc ^{2}(\eta)}{\alpha^{6} Q_{\alpha, 4}^{5}}+\frac{1224 \xi_{2}^{2} \xi_{3}^{2} a^{\prime}(t)^{4} \csc ^{2}(\eta)}{\alpha^{8} Q_{\alpha, 4}^{5}}+\frac{1224 \sec ^{2}(\eta) \xi_{3}^{2} \xi_{4}^{2} a^{\prime}(t)^{4} \csc ^{2}(\eta)}{\alpha^{8} Q_{\alpha, 4}^{5}}-\frac{8256 \sec ^{4}(\eta) \xi_{3}^{2} \xi_{4}^{4} a^{\prime}(t)^{4} \csc ^{2}(\eta)}{\alpha^{10} Q_{\alpha, 4}^{6}}+$ $\frac{480 \xi_{1}^{4} \xi_{3}^{2} a^{\prime}(t)^{4} \csc ^{2}(\eta)}{\alpha^{6} Q_{\alpha, 4}^{6}}-\frac{8256 \xi_{2}^{4} \xi_{3}^{2} a^{\prime}(t)^{4} \csc ^{2}(\eta)}{\alpha^{10} Q_{\alpha, 4}^{6}}-\frac{13920 \xi_{1}^{2} \xi_{2}^{2} \xi_{3}^{2} a^{\prime}(t)^{4} \csc ^{2}(\eta)}{\alpha^{8} Q_{\alpha, 4}^{6}}-\frac{13920 \sec ^{2}(\eta) \xi_{1}^{2} \xi_{3}^{2} \xi_{4}^{2} a^{\prime}(t)^{4} \csc ^{2}(\eta)}{\alpha^{8} Q_{\alpha, 4}^{6}}-$ $\frac{16512 \sec ^{2}(\eta) \xi_{2}^{2} \xi_{3}^{2} \xi_{4}^{2} a^{\prime}(t)^{4} \csc ^{2}(\eta)}{\alpha^{10} Q_{\alpha, 4}^{6}}+\frac{10240 \sec ^{6}(\eta) \xi_{3}^{2} \xi_{4}^{6} a^{\prime}(t)^{4} \csc ^{2}(\eta)}{\alpha^{12} Q_{\alpha, 4}^{7}}+\frac{141120 \sec ^{4}(\eta) \xi_{1}^{2} \xi_{3}^{2} \xi_{4}^{4} a^{\prime}(t)^{4} \csc ^{2}(\eta)}{\alpha^{10} Q_{\alpha, 4}^{7}}+$ $\frac{30720 \sec ^{4}(\eta) \xi_{2}^{2} \xi_{3}^{2} \xi_{4}^{4} a^{\prime}(t)^{4} \csc ^{2}(\eta)}{\alpha^{12} Q_{\alpha, 4}^{7}}+\frac{10240 \xi_{2}^{6} \xi_{3}^{2} a^{\prime}(t)^{4} \csc ^{2}(\eta)}{\alpha^{12} Q_{\alpha, 4}^{7}}+\frac{141120 \xi_{1}^{2} \xi_{2}^{4} \xi_{3}^{2} a^{\prime}(t)^{4} \csc ^{2}(\eta)}{\alpha^{10} Q_{\alpha, 4}^{7}}+\frac{17280 \xi_{1}^{4} \xi_{2}^{2} \xi_{3}^{2} a^{\prime}(t)^{4} \csc ^{2}(\eta)}{\alpha^{8} Q_{\alpha, 4}^{7}}+$ $\frac{17280 \sec ^{2}(\eta) \xi_{1}^{4} \xi_{3}^{2} \xi_{4}^{2} a^{\prime}(t)^{4} \csc ^{2}(\eta)}{\alpha^{8} Q^{7}}+\frac{30720 \sec ^{2}(\eta) \xi_{2}^{4} \xi_{3}^{2} \xi_{4}^{2} a^{\prime}(t)^{4} \csc ^{2}(\eta)}{\alpha^{12} Q^{7}}+\frac{282240 \sec ^{2}(\eta) \xi_{1}^{2} \xi_{2}^{2} \xi_{3}^{2} \xi_{4}^{2} a^{\prime}(t)^{4} \csc ^{2}(\eta)}{\alpha^{10} Q^{7}}-$ $\begin{array}{cc}\alpha^{8} Q_{\alpha, 4}^{7} & \alpha^{12} Q_{\alpha, 4}^{7} \\ 215040 \sec ^{6}(\eta) \xi_{1}^{2} \xi_{3}^{2} \xi_{4}^{6} a^{\prime}(t)^{4} \csc ^{2}(\eta)\end{array}-\frac{241920 \sec ^{4}(\eta) \xi_{1}^{4} \xi_{3}^{2} \xi_{4}^{4} a^{\prime}(t)^{4} \csc ^{2}(\eta)}{\alpha}-\frac{645120 \sec ^{4}(\eta) \xi_{1}^{2} \xi_{2}^{2} \xi_{3}^{2} \xi_{4}^{4} a^{\prime}(t)^{4} \csc ^{2}(\eta)}{\alpha}$ $\alpha^{12} Q_{\alpha, 4}^{8} \quad-\frac{\alpha^{10} Q_{\alpha, 4}^{8}}{\alpha^{12} Q_{\alpha, 4}^{8}}$

$\frac{215040 \xi_{1}^{2} \xi_{2}^{6} \xi_{3}^{2} a^{\prime}(t)^{4} \csc ^{2}(\eta)}{-}-\frac{241920 \xi_{1}^{4} \xi_{2}^{4} \xi_{3}^{2} a^{\prime}(t)^{4} \csc ^{2}(\eta)}{-645120 \sec ^{2}(\eta) \xi_{1}^{2} \xi_{2}^{4} \xi_{3}^{2} \xi_{4}^{2} a^{\prime}(t)^{4} \csc ^{2}(\eta)}-$ $\alpha^{12} Q_{\alpha, 4}^{8}-\frac{\alpha^{10} Q_{\alpha, 4}^{8}}{\alpha^{12} Q_{\alpha, 4}^{8}}$ $\frac{483840 \sec ^{2}(\eta) \xi_{1}^{4} \xi_{2}^{2} \xi_{3}^{2} \xi_{4}^{2} a^{\prime}(t)^{4} \csc ^{2}(\eta)}{\alpha^{10} Q^{8}}+\frac{430080 \sec ^{6}(\eta) \xi_{1}^{4} \xi_{3}^{2} \xi_{4}^{6} a^{\prime}(t)^{4} \csc ^{2}(\eta)}{\alpha^{12} Q^{9}}+\frac{1290240 \sec ^{4}(\eta) \xi_{1}^{4} \xi_{2}^{2} \xi_{3}^{2} \xi_{4}^{4} a^{\prime}(t)^{4} \csc ^{2}(\eta)}{\alpha^{12} Q^{9}}+$ $\alpha^{10} Q_{\alpha, 4}^{8}+\alpha^{12} Q^{9} \quad \alpha^{12} Q_{\alpha, 4}^{9}$

$\frac{430080 \xi_{1}^{4} \xi_{2}^{6} \xi_{3}^{2} a^{\prime}(t)^{4} \csc ^{2}(\eta)}{\alpha^{2} Q^{9}}+\frac{1290240 \sec ^{2}(\eta) \xi_{1}^{4} \xi_{2}^{4} \xi_{3}^{2} \xi_{4}^{2} a^{\prime}(t)^{4} \csc ^{2}(\eta)}{\alpha^{12} Q^{9}}-\frac{3 a^{\prime}(t)^{2} \csc ^{2}(\eta)}{\alpha^{4}}-\frac{3 \xi_{1}^{2} a^{\prime}(t)^{2} \csc ^{2}(\eta)}{\alpha^{4} Q^{4}}+$ $\alpha^{12} Q_{\alpha, 4}^{9}-\frac{\alpha^{12} Q_{\alpha, 4}^{9}}{\alpha^{4} Q_{\alpha, 4}^{3}}-\frac{3 \xi^{2}}{\alpha^{4} Q_{\alpha, 4}^{4}}$

$\frac{13 \xi_{2}^{2} a^{\prime}(t)^{2} \csc ^{2}(\eta)}{\alpha^{6} Q^{4}}-\frac{87 \cot ^{2}(\eta) \xi_{3}^{2} a^{\prime}(t)^{2} \csc ^{2}(\eta)}{\alpha^{6} Q^{4}}+\frac{13 \sec ^{2}(\eta) \xi_{3}^{2} a^{\prime}(t)^{2} \csc ^{2}(\eta)}{\alpha^{6} Q^{4}}+\frac{116 \cot (\eta) \cot (2 \eta) \xi_{3}^{2} a^{\prime}(t)^{2} \csc ^{2}(\eta)}{\alpha^{6} Q^{4}}+$ $\frac{52 \xi_{3}^{2} a^{\prime}(t)^{2} \csc ^{2}(\eta)}{\alpha^{6} Q^{4}}+\frac{13 \sec ^{2}(\eta) \xi_{4}^{2} a^{\prime}(t)^{2} \csc ^{2}(\eta)}{\alpha^{6} Q^{4}}-\frac{32 \xi_{2}^{4} a^{\prime}(t)^{2} \csc ^{2}(\eta)}{\alpha^{8} Q^{5}}-\frac{32 \sec ^{4}(\eta) \xi_{4}^{4} a^{\prime}(t)^{2} \csc ^{2}(\eta)}{\alpha^{8} Q^{5}}-\frac{48 \xi_{1}^{2} \xi_{2}^{2} a^{\prime}(t)^{2} \csc ^{2}(\eta)}{\alpha^{6} Q^{5}}+$ $\frac{216 \cot ^{2}(\eta) \xi_{1}^{2} \xi_{3}^{2} a^{\prime}(t)^{2} \csc ^{2}(\eta)}{\alpha^{6} Q^{5}}-\frac{48 \sec ^{2}(\eta) \xi_{1}^{2} \xi_{3}^{2} a^{\prime}(t)^{2} \csc ^{2}(\eta)}{\alpha^{6} Q^{5}}-\frac{288 \cot (\eta) \cot (2 \eta) \xi_{1}^{2} \xi_{3}^{2} a^{\prime}(t)^{2} \csc ^{2}(\eta)}{\alpha^{6} Q^{5}}-\frac{192 \xi_{1}^{2} \xi_{3}^{2} a^{\prime}(t)^{2} \csc ^{2}(\eta)}{\alpha^{6} Q^{5}}+$ $\frac{2552 \cot ^{2}(\eta) \xi_{2}^{2} \xi_{3}^{2} a^{\prime}(t)^{2} \csc ^{2}(\eta)}{\alpha^{8} Q^{5}}+\frac{928 \cot ^{2}(2 \eta) \xi_{2}^{2} \xi_{3}^{2} a^{\prime}(t)^{2} \csc ^{2}(\eta)}{a^{8} Q^{5}}-\frac{1856 \csc ^{2}(2 \eta) \xi_{2}^{2} \xi_{3}^{2} a^{\prime}(t)^{2} \csc ^{2}(\eta)}{a^{8} Q^{5}}-$

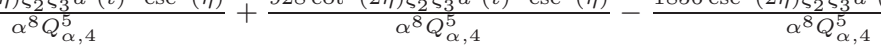
$\frac{64 \sec ^{2}(\eta) \xi_{2}^{2} \xi_{3}^{2} a^{\prime}(t)^{2} \csc ^{2}(\eta)}{\alpha^{8} Q_{\alpha, 4}^{5}}-\frac{3712 \cot (\eta) \cot (2 \eta) \xi_{2}^{2} \xi_{3}^{2} a^{\prime}(t)^{2} \csc ^{2}(\eta)}{\alpha^{8} Q^{5}}-\frac{256 \xi_{2}^{2} \xi_{3}^{2} a^{\prime}(t)^{2} \csc ^{2}(\eta)}{\alpha^{8} Q^{5}}-\frac{48 \sec ^{2}(\eta) \xi_{1}^{2} \xi_{4}^{2} a^{\prime}(t)^{2} \csc ^{2}(\eta)}{\alpha^{6} Q^{5}}-$ $\frac{64 \sec ^{2}(\eta) \xi_{2}^{2} \xi_{4}^{2} a^{\prime}(t)^{2} \csc ^{2}(\eta)}{\alpha^{8} Q_{\alpha, 4}^{5}}+\frac{400 \sec ^{4}(\eta) \xi_{3}^{2} \xi_{4}^{2} a^{\prime}(t)^{2} \csc ^{2}(\eta)}{\alpha^{8} Q_{\alpha, 4}^{5}}-\frac{2112 \sec ^{2}(\eta) \xi_{3}^{2} \xi_{4}^{2} a^{\prime}(t)^{2} \csc ^{2}(\eta)}{\alpha^{8} Q_{\alpha, 4}^{5}}+\frac{240 \xi_{1}^{2} \xi_{2}^{4} a^{\prime}(t)^{2} \csc ^{2}(\eta)}{\alpha^{8} Q_{\alpha, 4}^{6}}+$ $\frac{240 \sec ^{4}(\eta) \xi_{1}^{2} \xi_{4}^{4} a^{\prime}(t)^{2} \csc ^{2}(\eta)}{\alpha^{8} Q_{\alpha, 4}^{6}}-\frac{1280 \sec ^{6}(\eta) \xi_{3}^{2} \xi_{4}^{4} a^{\prime}(t)^{2} \csc ^{2}(\eta)}{\alpha^{10} Q_{\alpha, 4}^{6}}+\frac{9600 \sec ^{4}(\eta) \xi_{3}^{2} \xi_{4}^{4} a^{\prime}(t)^{2} \csc ^{2}(\eta)}{\alpha^{10} Q_{\alpha, 4}^{6}}-$ $\frac{10560 \cot ^{2}(\eta) \xi_{2}^{4} \xi_{3}^{2} a^{\prime}(t)^{2} \csc ^{2}(\eta)}{\alpha^{10} Q_{\alpha, 4}^{6}}-\frac{2560 \cot ^{2}(2 \eta) \xi_{2}^{4} \xi_{3}^{2} a^{\prime}(t)^{2} \csc ^{2}(\eta)}{\alpha^{10} Q_{\alpha, 4}^{6}}+\frac{5120 \csc ^{2}(2 \eta) \xi_{2}^{4} \xi_{3}^{2} a^{\prime}(t)^{2} \csc ^{2}(\eta)}{\alpha^{10} Q_{\alpha, 4}^{6}}+$ $\frac{15680 \cot (\eta) \cot (2 \eta) \xi_{2}^{4} \xi_{3}^{2} a^{\prime}(t)^{2} \csc ^{2}(\eta)}{\alpha^{10} Q_{\alpha, 4}^{6}}-\frac{13920 \cot ^{2}(\eta) \xi_{1}^{2} \xi_{2}^{2} \xi_{3}^{2} a^{\prime}(t)^{2} \csc ^{2}(\eta)}{\alpha^{8} Q_{\alpha, 4}^{6}}-\frac{5760 \cot ^{2}(2 \eta) \xi_{1}^{2} \xi_{2}^{2} \xi_{3}^{2} a^{\prime}(t)^{2} \csc ^{2}(\eta)}{\alpha^{8} Q_{\alpha, 4}^{6}}+$ $\frac{11520 \csc ^{2}(2 \eta) \xi_{1}^{2} \xi_{2}^{2} \xi_{3}^{2} a^{\prime}(t)^{2} \csc ^{2}(\eta)}{\alpha^{8} Q_{\alpha, 4}^{6}}+\frac{480 \sec ^{2}(\eta) \xi_{1}^{2} \xi_{2}^{2} \xi_{3}^{2} a^{\prime}(t)^{2} \csc ^{2}(\eta)}{\alpha^{8} Q^{6}}+\frac{20160 \cot (\eta) \cot (2 \eta) \xi_{1}^{2} \xi_{2}^{2} \xi_{3}^{2} a^{\prime}(t)^{2} \csc ^{2}(\eta)}{\alpha^{8} Q^{6}}+$ $\frac{1920 \xi_{1}^{2} \xi_{2}^{2} \xi_{3}^{2} a^{\prime}(t)^{2} \csc ^{2}(\eta)}{\alpha^{8} Q_{\alpha, 4}^{6}}-\frac{960 \sec ^{4}(\eta) \xi_{3}^{4} \xi_{4}^{2} a^{\prime}(t)^{2} \csc ^{2}(\eta)}{\alpha^{10} Q_{\alpha, 4}^{6}}+\frac{480 \sec ^{2}(\eta) \xi_{1}^{2} \xi_{2}^{2} \xi_{4}^{2} a^{\prime}(t)^{2} \csc ^{2}(\eta)}{\alpha^{8} Q_{\alpha, 4}^{6}}-\frac{2400 \sec ^{4}(\eta) \xi_{1}^{2} \xi_{3}^{2} \xi_{4}^{2} a^{\prime}(t)^{2} \csc ^{2}(\eta)}{\alpha^{8} Q_{\alpha, 4}^{6}}+$ $\frac{11520 \sec ^{2}(\eta) \xi_{1}^{2} \xi_{3}^{2} \xi_{4}^{2} a^{\prime}(t)^{2} \csc ^{2}(\eta)}{8}-\frac{6080 \sec ^{4}(\eta) \xi_{2}^{2} \xi_{3}^{2} \xi_{4}^{2} a^{\prime}(t)^{2} \csc ^{2}(\eta)}{\alpha^{10} Q^{6}}-\frac{2560 \cot ^{2}(2 \eta) \sec ^{2}(\eta) \xi_{2}^{2} \xi_{3}^{2} \xi_{4}^{2} a^{\prime}(t)^{2} \csc ^{2}(\eta)}{\alpha^{10} Q^{6}}+$

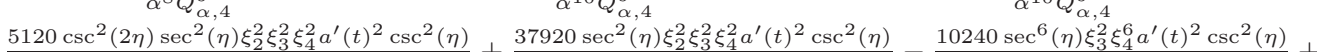
$\frac{5120 \csc ^{2}(2 \eta) \sec ^{2}(\eta) \xi_{2} \xi_{3} \xi_{4}^{2} a^{\prime}(t)^{2} \csc ^{2}(\eta)}{\alpha^{10} Q_{\alpha}^{6}}+\frac{37920 \sec ^{2}(\eta) \xi_{2} \xi_{3} \xi_{4}^{2} a^{\prime}(t)^{2} \csc ^{2}(\eta)}{\alpha^{10} Q_{\alpha, 4}^{6}}-\frac{10240 \sec ^{6}(\eta) \xi_{3}^{2} \xi_{4}^{6} a^{\prime}(t)^{2} \csc ^{2}(\eta)}{\alpha^{12} Q_{\alpha, 4}^{7}}+$ $\frac{5120 \sec ^{6}(\eta) \xi_{3}^{4} \xi_{4}^{4} a^{\prime}(t)^{2} \csc ^{2}(\eta)}{11520 \sec ^{6}(\eta) \xi_{1}^{2} \xi_{3}^{2} \xi_{4}^{4} a^{\prime}(t)^{2} \csc ^{2}(\eta)}-\frac{76800 \sec ^{4}(\eta) \xi_{1}^{2} \xi_{3}^{2} \xi_{4}^{4} a^{\prime}(t)^{2} \csc ^{2}(\eta)}{\alpha^{10} Q_{\alpha, 4}^{7}}+$ $\alpha^{12} Q_{\alpha, 4}^{7}+\frac{11520}{\alpha^{10} Q_{\alpha, 4}^{7}}-\frac{\alpha^{10} Q_{\alpha, 4}^{7}}{137600 a^{\prime}}$

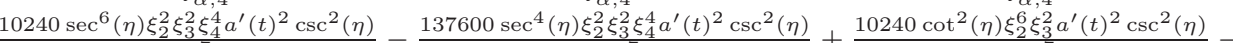

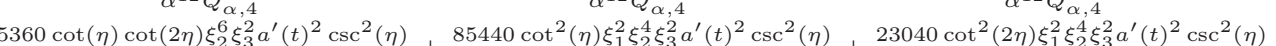

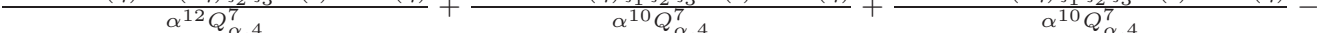
$\frac{46080 \csc ^{2}(2 \eta) \xi_{1}^{2} \xi_{2}^{4} \xi_{3}^{2} a^{\prime}(t)^{2} \csc ^{2}(\eta)}{\alpha^{10} Q^{7}}-\frac{126720 \cot (\eta) \cot (2 \eta) \xi_{1}^{2} \xi_{2}^{4} \xi_{3}^{2} a^{\prime}(t)^{2} \csc ^{2}(\eta)}{\alpha^{10} Q^{7}}+\frac{8640 \sec ^{4}(\eta) \xi_{1}^{2} \xi_{3}^{4} \xi_{4}^{2} a^{\prime}(t)^{2} \csc ^{2}(\eta)}{\alpha^{10} Q^{7}}+$ $\frac{10240 \sec ^{4}(\eta) \xi_{2}^{2} \xi_{3}^{4} \xi_{4}^{2} a^{\prime}(t)^{2} \csc ^{2}(\eta)}{\alpha^{12} Q^{7}}+\frac{10240 \sec ^{4}(\eta) \xi_{2}^{4} \xi_{3}^{2} \xi_{4}^{2} a^{\prime}(t)^{2} \csc ^{2}(\eta)}{\alpha^{12} Q^{7}}-\frac{127360 \sec ^{2}(\eta) \xi_{2}^{4} \xi_{3}^{2} \xi_{4}^{2} a^{\prime}(t)^{2} \csc ^{2}(\eta)}{\alpha^{12} Q^{7}}+$ $\frac{49920 \sec ^{4}(\eta) \xi_{1}^{2} \xi_{2}^{2} \xi_{3}^{2} \xi_{4}^{2} a^{\prime}(t)^{2} \csc ^{2}(\eta)}{\alpha^{10} Q^{7}}+\frac{23040 \cot ^{2}(2 \eta) \sec ^{2}(\eta) \xi_{1}^{2} \xi_{2}^{2} \xi_{3}^{2} \xi_{4}^{2} a^{\prime}(t)^{2} \csc ^{2}(\eta)}{\alpha^{10} Q^{7}}-$ $\frac{46080 \csc ^{2}(2 \eta) \sec ^{2}(\eta) \xi_{1}^{2} \xi_{2}^{2} \xi_{3}^{2} \xi_{4}^{2} a^{\prime}(t)^{2} \csc ^{2}(\eta)}{\alpha^{10} Q^{7}}-\frac{278400 \sec ^{2}(\eta) \xi_{1}^{2} \xi_{2}^{2} \xi_{3}^{2} \xi_{4}^{2} a^{\prime}(t)^{2} \csc ^{2}(\eta)}{\alpha^{10} Q_{\alpha, 4}^{7}}+\frac{107520 \sec ^{6}(\eta) \xi_{1}^{2} \xi_{3}^{2} \xi_{4}^{6} a^{\prime}(t)^{2} \csc ^{2}(\eta)}{\alpha^{12} Q_{\alpha, 4}^{8}}+$ 


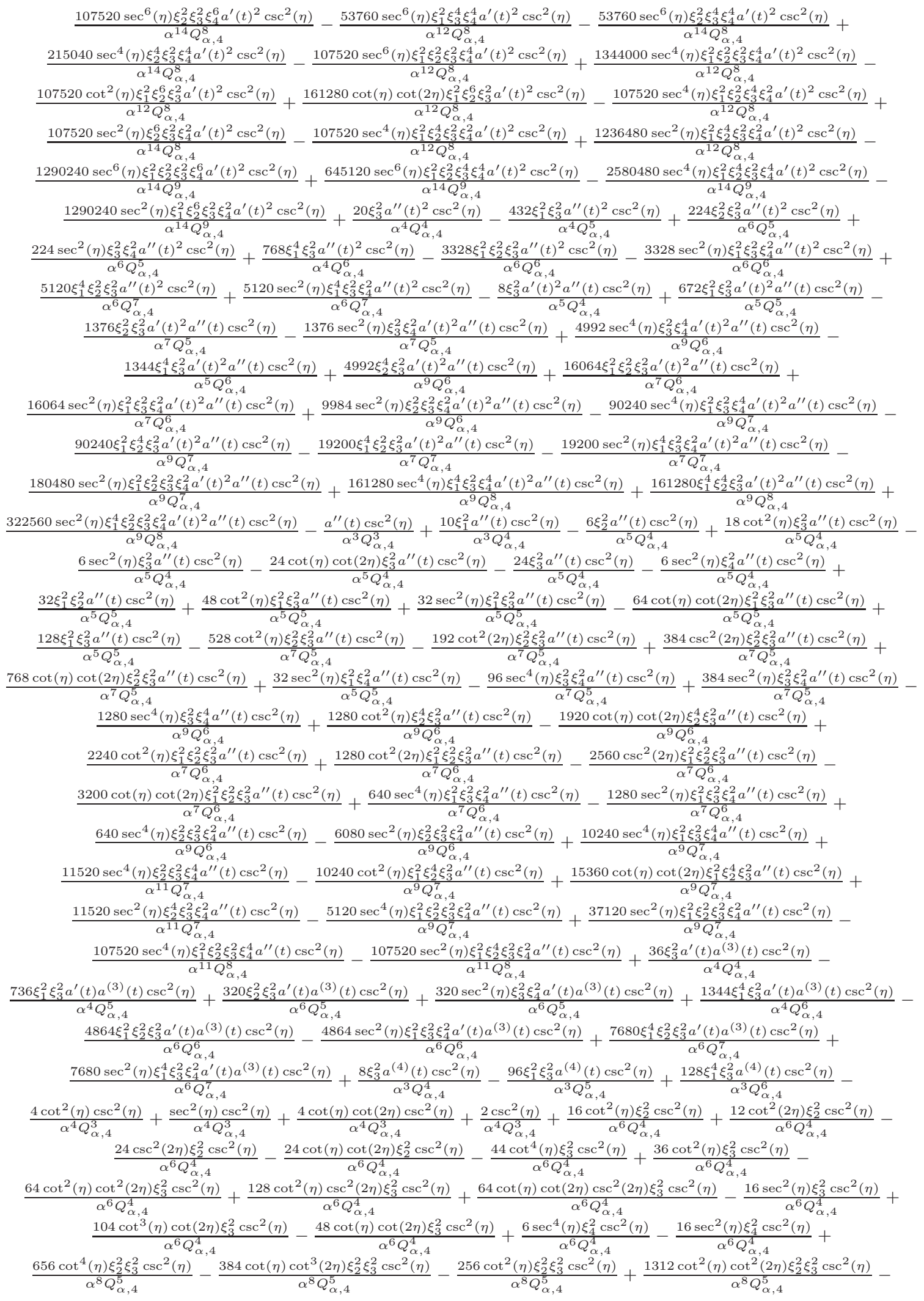


$\frac{2624 \cot ^{2}(\eta) \csc ^{2}(2 \eta) \xi_{2}^{2} \xi_{3}^{2} \csc ^{2}(\eta)}{\alpha^{8} Q^{5}}-\frac{1536 \cot (\eta) \cot (2 \eta) \csc ^{2}(2 \eta) \xi_{2}^{2} \xi_{3}^{2} \csc ^{2}(\eta)}{\alpha^{8} Q^{5}}+\frac{96 \sec ^{2}(\eta) \xi_{2}^{2} \xi_{3}^{2} \csc ^{2}(\eta)}{\alpha^{8} Q^{5} 4^{4}}-$ $\frac{1600 \cot ^{3}(\eta) \cot (2 \eta) \xi_{2}^{2} \xi_{3}^{2} \csc ^{2}(\eta)}{\alpha^{8} Q^{5}}+\frac{384 \cot (\eta) \cot (2 \eta) \xi_{2}^{2} \xi_{3}^{2} \csc ^{2}(\eta)}{\alpha^{8} Q^{5}}-\frac{32 \sec ^{4}(\eta) \xi_{2}^{2} \xi_{4}^{2} \csc ^{2}(\eta)}{\alpha^{8} Q^{5}}+\frac{96 \sec ^{2}(\eta) \xi_{2}^{2} \xi_{4}^{2} \csc ^{2}(\eta)}{\alpha^{8} Q^{5}}-$ $\frac{688 \sec ^{4}(\eta) \xi_{3}^{2} \xi_{4}^{2} \csc ^{2}(\eta)}{\alpha^{8} Q^{5}}-\frac{384 \cot ^{2}(2 \eta) \sec ^{2}(\eta) \xi_{3}^{2} \xi_{4}^{2} \csc ^{2}(\eta)}{\alpha^{8} Q^{5}}+\frac{768 \csc ^{2}(2 \eta) \sec ^{2}(\eta) \xi_{3}^{2} \xi_{4}^{2} \csc ^{2}(\eta)}{\alpha^{8} Q^{5}}+\frac{968 \sec ^{2}(\eta) \xi_{3}^{2} \xi_{4}^{2} \csc ^{2}(\eta)}{\alpha^{8} Q^{5}}+$ $\frac{1664 \sec ^{6}(\eta) \xi_{3}^{2} \xi_{4}^{4} \csc ^{2}(\eta)}{\alpha^{10} Q_{\alpha, 4}^{6}}-\frac{3008 \sec ^{4}(\eta) \xi_{3}^{2} \xi_{4}^{4} \csc ^{2}(\eta)}{\alpha^{10} Q_{\alpha, 4}^{6}}-\frac{1024 \cot ^{4}(\eta) \xi_{2}^{4} \xi_{3}^{2} \csc ^{2}(\eta)}{\alpha^{10} Q_{\alpha, 4}^{6}}+\frac{1280 \cot (\eta) \cot ^{3}(2 \eta) \xi_{2}^{4} \xi_{3}^{2} \csc ^{2}(\eta)}{\alpha^{10} Q_{\alpha, 4}^{6}}-$ $\frac{2560 \cot ^{2}(\eta) \cot ^{2}(2 \eta) \xi_{2}^{4} \xi_{3}^{2} \csc ^{2}(\eta)}{\alpha^{10} Q_{\alpha, 4}^{6}}+\frac{5120 \cot ^{2}(\eta) \csc ^{2}(2 \eta) \xi_{2}^{4} \xi_{3}^{2} \csc ^{2}(\eta)}{\alpha^{10} Q_{\alpha, 4}^{6}}+\frac{2560 \cot (\eta) \cot (2 \eta) \csc ^{2}(2 \eta) \xi_{2}^{4} \xi_{3}^{2} \csc ^{2}(\eta)}{\alpha^{10} Q_{\alpha, 4}^{6}}+$ $\frac{2560 \cot ^{3}(\eta) \cot (2 \eta) \xi_{2}^{4} \xi_{3}^{2} \csc ^{2}(\eta)}{\alpha^{10} Q_{\alpha, 4}^{6}}+\frac{11680 \sec ^{4}(\eta) \xi_{2}^{2} \xi_{3}^{2} \xi_{4}^{2} \csc ^{2}(\eta)}{\alpha^{10} Q_{\alpha, 4}^{6}}+\frac{10240 \cot ^{2}(2 \eta) \sec ^{2}(\eta) \xi_{2}^{2} \xi_{3}^{2} \xi_{4}^{2} \csc ^{2}(\eta)}{\alpha^{10} Q_{\alpha, 4}^{6}}-$ $\frac{20480 \csc ^{2}(2 \eta) \sec ^{2}(\eta) \xi_{2}^{2} \xi_{3}^{2} \xi_{4}^{2} \csc ^{2}(\eta)}{\alpha^{10} Q_{\alpha, 4}^{6}}-\frac{13952 \sec ^{2}(\eta) \xi_{2}^{2} \xi_{3}^{2} \xi_{4}^{2} \csc ^{2}(\eta)}{\alpha^{10} Q_{\alpha, 4}^{6}}-\frac{30080 \sec ^{6}(\eta) \xi_{2}^{2} \xi_{3}^{2} \xi_{4}^{4} \csc ^{2}(\eta)}{\alpha^{12} Q_{\alpha, 4}^{7}}+\frac{57280 \sec ^{4}(\eta) \xi_{2}^{2} \xi_{3}^{2} \xi_{4}^{4} \csc ^{2}(\eta)}{\alpha^{12} Q_{\alpha, 4}^{7}}-$

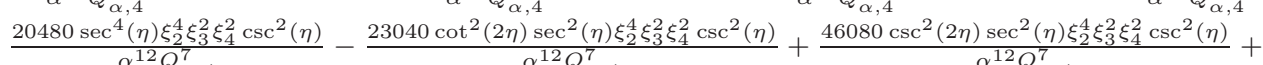

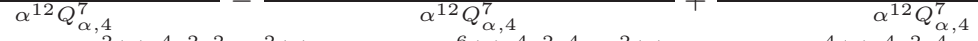
$\frac{20480 \sec ^{2}(\eta) \xi_{2}^{4} \xi_{3}^{2} \xi_{4}^{2} \csc ^{2}(\eta)}{\alpha^{12} Q_{\alpha, 4}^{7}}+\frac{53760 \sec ^{6}(\eta) \xi_{2}^{4} \xi_{3}^{2} \xi_{4}^{4} \csc ^{2}(\eta)}{\alpha^{14} Q_{\alpha, 4}^{8}}-\frac{107520 \sec ^{4}(\eta) \xi_{2}^{4} \xi_{3}^{2} \xi_{4}^{4} \csc ^{2}(\eta)}{\alpha^{14} Q_{\alpha, 4}^{8}}+$ $\frac{928 \cot (2 \eta) \sec ^{3}(\eta) \xi_{3}^{2} \xi_{4}^{2} a^{\prime}(t)^{2} \csc (\eta)}{2560 \cot (2 \eta) \sec ^{5}(\eta) \xi_{3}^{2} \xi_{4}^{4} a^{\prime}(t)^{2} \csc (\eta)}-\frac{5760 \cot (2 \eta) \sec ^{3}(\eta) \xi_{1}^{2} \xi_{3}^{2} \xi_{4}^{2} a^{\prime}(t)^{2} \csc (\eta)}{\alpha^{6} Q^{6}}-$ $\alpha^{8} Q_{\alpha, 4}^{5}-\frac{\alpha^{10} Q_{\alpha, 4}^{6}}{\alpha^{8} Q_{\alpha, 4}^{6}}$ $\underline{16960 \cot (2 \eta) \sec ^{3}(\eta) \xi_{2}^{2} \xi_{3}^{2} \xi_{4}^{2} a^{\prime}(t)^{2} \csc (\eta)}+\frac{23040 \cot (2 \eta) \sec ^{5}(\eta) \xi_{1}^{2} \xi_{3}^{2} \xi_{4}^{4} a^{\prime}(t)^{2} \csc (\eta)}{30720 \cot (2 \eta) \sec ^{5}(\eta) \xi_{2}^{2} \xi_{3}^{2} \xi_{4}^{4} a^{\prime}(t)^{2} \csc (\eta)}+$ ${ }^{10} Q_{\alpha, 4}^{6} \quad{ }^{\alpha 10} Q_{\alpha, 4}^{7} \quad{ }^{\alpha^{12} Q_{\alpha, 4}^{*}}$ $\frac{30720 \cot (2 \eta) \sec ^{3}(\eta) \xi_{2}^{4} \xi_{3}^{2} \xi_{4}^{2} a^{\prime}(t)^{2} \csc (\eta)}{\alpha^{12} Q_{\alpha, 4}^{7}}+\frac{138240 \cot (2 \eta) \sec ^{3}(\eta) \xi_{1}^{2} \xi_{2}^{2} \xi_{3}^{2} \xi_{4}^{2} a^{\prime}(t)^{2} \csc (\eta)}{\alpha^{10} Q_{\alpha, 4}^{7}}-$

$\frac{322560 \cot (2 \eta) \sec ^{5}(\eta) \xi_{1}^{2} \xi_{2}^{2} \xi_{3}^{2} \xi_{4}^{4} a^{\prime}(t)^{2} \csc (\eta)}{\alpha^{12} Q^{8}}-\frac{322560 \cot (2 \eta) \sec ^{3}(\eta) \xi_{1}^{2} \xi_{2}^{4} \xi_{3}^{2} \xi_{4}^{2} a^{\prime}(t)^{2} \csc (\eta)}{\alpha^{12} Q^{8}}-\frac{192 \cot (2 \eta) \sec ^{3}(\eta) \xi_{3}^{2} \xi_{4}^{2} a^{\prime \prime}(t) \csc (\eta)}{\alpha^{7} Q^{5}}+$ $\frac{1280 \cot (2 \eta) \sec ^{3}(\eta) \xi_{1}^{2} \xi_{3}^{2} \xi_{4}^{2} a^{\prime \prime}(t) \csc (\eta)}{a^{7} Q^{6}}+\frac{1920 \cot (2 \eta) \sec ^{3}(\eta) \xi_{2}^{2} \xi_{3}^{2} \xi_{4}^{2} a^{\prime \prime}(t) \csc (\eta)}{\alpha^{9} Q^{6}}-\frac{15360 \cot (2 \eta) \sec ^{3}(\eta) \xi_{1}^{2} \xi_{2}^{2} \xi_{3}^{2} \xi_{4}^{2} a^{\prime \prime}(t) \csc (\eta)}{\alpha^{9} Q^{7}}+$ $\frac{12 \cot (2 \eta) \sec ^{3}(\eta) \xi_{4}^{2} \csc (\eta)}{\alpha^{6} Q_{\alpha, 4}^{4}}-\frac{96 \cot (2 \eta) \sec ^{3}(\eta) \xi_{2}^{2} \xi_{4}^{2} \csc (\eta)}{{ }^{8} Q^{5}}-\frac{928 \cot (2 \eta) \sec ^{3}(\eta) \xi_{3}^{2} \xi_{4}^{2} \csc (\eta)}{\alpha^{8} Q^{5}}+\frac{3840 \cot (2 \eta) \sec ^{5}(\eta) \xi_{3}^{2} \xi_{4}^{4} \csc (\eta)}{\alpha^{10} Q_{\alpha}^{6}}+$ $\alpha^{6} Q_{\alpha, 4}^{4}-\frac{\alpha^{8} Q_{\alpha, 4}^{5}}{\alpha^{8} Q_{\alpha, 4}^{5}}-\frac{38 \xi^{3}{ }^{10} Q_{\alpha, 4}^{6}}{\alpha^{5}}$ $\frac{16960 \cot (2 \eta) \sec ^{3}(\eta) \xi_{2}^{2} \xi_{3}^{2} \xi_{4}^{2} \csc (\eta)}{\alpha^{10} Q^{6}}-\frac{80640 \cot (2 \eta) \sec ^{5}(\eta) \xi_{2}^{2} \xi_{3}^{2} \xi_{4}^{4} \csc (\eta)}{\alpha^{12} Q^{7}}-\frac{30720 \cot (2 \eta) \sec ^{3}(\eta) \xi_{2}^{4} \xi_{3}^{2} \xi_{4}^{2} \csc (\eta)}{\alpha^{12} Q^{7}}+$ $\frac{161280 \cot (2 \eta) \sec ^{5}(\eta) \xi_{2}^{4} \xi_{3}^{2} \xi_{4}^{4} \csc (\eta)}{\alpha^{14} Q^{8}}-\frac{44 \sec ^{2}(\eta) \xi_{4}^{2} \tan ^{4}(\eta)}{\alpha^{6} Q^{4}}+\frac{868 \sec ^{4}(\eta) \xi_{4}^{4} \tan ^{4}(\eta)}{\alpha^{8} Q^{5}}+\frac{656 \sec ^{2}(\eta) \xi_{2}^{2} \xi_{4}^{2} \tan ^{4}(\eta)}{\alpha^{8} Q^{5}}-$ $\frac{3008 \sec ^{6}(\eta) \xi_{4}^{6} \tan ^{4}(\eta)}{\alpha^{10} Q^{6}}-\frac{14784 \sec ^{4}(\eta) \xi_{2}^{2} \xi_{4}^{4} \tan ^{4}(\eta)}{\alpha^{10} Q_{\alpha, 4}^{6}}-\frac{1024 \sec ^{2}(\eta) \xi_{2}^{4} \xi_{4}^{2} \tan ^{4}(\eta)}{\alpha^{10} Q_{\alpha, 4}^{6}}+\frac{2560 \sec ^{8}(\eta) \xi_{4}^{8} \tan ^{4}(\eta)}{\alpha^{12} Q_{\alpha, 4}^{7}}+$ $\frac{57280 \sec ^{6}(\eta) \xi_{2}^{2} \xi_{4}^{6} \tan ^{4}(\eta)}{\alpha^{12} Q_{\alpha}^{7}}+\frac{25600 \sec ^{4}(\eta) \xi_{2}^{4} \xi_{4}^{4} \tan ^{4}(\eta)}{\alpha^{12} Q_{\alpha}^{7}}-\frac{53760 \sec ^{8}(\eta) \xi_{2}^{2} \xi_{4}^{8} \tan ^{4}(\eta)}{\alpha^{14} Q_{\alpha}^{8}}-\frac{107520 \sec ^{6}(\eta) \xi_{2}^{4} \xi_{4}^{6} \tan ^{4}(\eta)}{\alpha^{14} Q_{\alpha, 4}^{8}}+$ $\frac{107520 \sec ^{8}(\eta) \xi_{2}^{4} \xi_{4}^{8} \tan ^{4}(\eta)}{\alpha^{16} Q_{\alpha, 4}^{9}}+\frac{9 a^{\prime}(t)^{4}}{\alpha^{4} Q_{\alpha, 4}^{3}}-\frac{27 \xi_{1}^{2} a^{\prime}(t)^{4}}{\alpha^{4} Q_{\alpha, 4}^{4}}-\frac{7 \xi_{2}^{2} a^{\prime}(t)^{4}}{\alpha^{6} Q_{\alpha, 4}^{4}}-\frac{7 \sec ^{2}(\eta) \xi_{4}^{2} a^{\prime}(t)^{4}}{\alpha^{6} Q_{\alpha, 4}^{4}}+\frac{36 \xi_{1}^{4} a^{\prime}(t)^{4}}{\alpha^{4} Q_{\alpha, 4}^{5}}+\frac{612 \xi_{2}^{4} a^{\prime}(t)^{4}}{\alpha^{8} Q_{\alpha, 4}^{5}}+$ $\frac{612 \sec ^{4}(\eta) \xi_{4}^{4} a^{\prime}(t)^{4}}{\alpha^{8} Q_{\alpha, 4}^{5}}-\frac{216 \xi_{1}^{2} \xi_{2}^{2} a^{\prime}(t)^{4}}{\alpha^{6} Q_{\alpha, 4}^{5}}-\frac{216 \sec ^{2}(\eta) \xi_{1}^{2} \xi_{4}^{2} a^{\prime}(t)^{4}}{\alpha^{6} Q_{\alpha, 4}^{5}}+\frac{1224 \sec ^{2}(\eta) \xi_{2}^{2} \xi_{4}^{2} a^{\prime}(t)^{4}}{\alpha^{8} Q_{\alpha, 4}^{5}}-\frac{2752 \xi_{2}^{6} a^{\prime}(t)^{4}}{\alpha^{10} Q_{\alpha, 4}^{6}}-\frac{2752 \sec ^{6}(\eta) \xi_{4}^{6} a^{\prime}(t)^{4}}{\alpha^{10} Q_{\alpha, 4}^{6}}-$ $\frac{6960 \xi_{1}^{2} \xi_{2}^{4} a^{\prime}(t)^{4}}{\alpha^{8} Q_{\alpha, 4}}-\frac{6960 \sec ^{4}(\eta) \xi_{1}^{2} \xi_{4}^{4} a^{\prime}(t)^{4}}{2}-\frac{8256 \sec ^{4}(\eta) \xi_{2}^{2} \xi_{4}^{4} a^{\prime}(t)^{4}}{{ }^{10} Q_{\alpha, 4}^{6}}+\frac{480 \xi_{1}^{4} \xi_{2}^{2} a^{\prime}(t)^{4}}{\alpha^{6} Q_{\alpha, 4}}+\frac{480 \sec ^{2}(\eta) \xi_{1}^{4} \xi_{4}^{2} a^{\prime}(t)^{4}}{\alpha^{6} Q_{\alpha}}-$ $\alpha^{8} Q_{\alpha, 4}^{6}-\frac{\alpha^{8} Q_{\alpha, 4}^{6}}{\alpha^{10} Q_{\alpha, 4}^{6}}+\frac{\alpha^{6}}{\alpha^{6} Q_{\alpha, 4}^{6}}+\frac{480 a^{6}}{\alpha^{6} Q_{\alpha, 4}^{6}}$ $\frac{8256 \sec ^{2}(\eta) \xi_{2}^{4} \xi_{4}^{2} a^{\prime}(t)^{4}}{\alpha^{10} Q_{\alpha, 4}^{6}}-\frac{13920 \sec ^{2}(\eta) \xi_{1}^{2} \xi_{2}^{2} \xi_{4}^{2} a^{\prime}(t)^{4}}{\alpha^{8} Q_{\alpha, 4}^{6}}+\frac{2560 \xi_{2}^{8} a^{\prime}(t)^{4}}{\alpha^{12} Q_{\alpha, 4}^{7}}+\frac{2560 \sec ^{8}(\eta) \xi_{4}^{8} a^{\prime}(t)^{4}}{\alpha^{12} Q_{\alpha, 4}^{7}}+\frac{47040 \xi_{1}^{2} \xi_{2}^{6} a^{\prime}(t)^{4}}{\alpha^{10} Q_{\alpha, 4}^{7}}+$ $\frac{47040 \sec ^{6}(\eta) \xi_{1}^{2} \xi_{4}^{6} a^{\prime}(t)^{4}}{\alpha^{10} Q_{\alpha, 4}^{7}}+\frac{10240 \sec ^{6}(\eta) \xi_{2}^{2} \xi_{4}^{6} a^{\prime}(t)^{4}}{\alpha^{12} Q_{\alpha, 4}^{7}}+\frac{8640 \xi_{1}^{4} \xi_{2}^{4} a^{\prime}(t)^{4}}{\alpha^{8} Q_{\alpha, 4}^{7}}+\frac{8640 \sec ^{4}(\eta) \xi_{1}^{4} \xi_{4}^{4} a^{\prime}(t)^{4}}{\alpha^{8} Q_{\alpha, 4}^{7}}+\frac{15360 \sec ^{4}(\eta) \xi_{2}^{4} \xi_{4}^{4} a^{\prime}(t)^{4}}{\alpha^{12} Q_{\alpha, 4}^{7}}+$ $\frac{141120 \sec ^{4}(\eta) \xi_{1}^{2} \xi_{2}^{2} \xi_{4}^{4} a^{\prime}(t)^{4}}{10}+\frac{10240 \sec ^{2}(\eta) \xi_{2}^{6} \xi_{4}^{2} a^{\prime}(t)^{4}}{\alpha^{12} Q_{\alpha, 4}}+\frac{141120 \sec ^{2}(\eta) \xi_{1}^{2} \xi_{2}^{4} \xi_{4}^{2} a^{\prime}(t)^{4}}{\alpha^{10} Q^{1}}+\frac{17280 \sec ^{2}(\eta) \xi_{1}^{4} \xi_{2}^{2} \xi_{4}^{2} a^{\prime}(t)^{4}}{\alpha^{8} Q_{\alpha, 4}}$

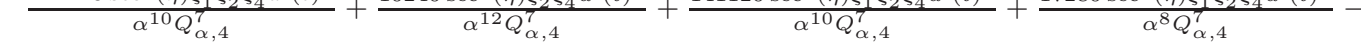
$\frac{53760 \xi_{1}^{2} \xi_{2}^{8} a^{\prime}(t)^{4}}{\alpha^{12}}-\frac{53760 \sec ^{8}(\eta) \xi_{1}^{2} \xi_{4}^{8} a^{\prime}(t)^{4}}{a^{12} Q_{\alpha, 4}}-\frac{80640 \xi_{1}^{4} \xi_{2}^{6} a^{\prime}(t)^{4}}{Q_{\alpha}}-\frac{80640 \sec ^{6}(\eta) \xi_{1}^{4} \xi_{4}^{6} a^{\prime}(t)^{4}}{\alpha_{\alpha}^{10}}-\frac{215040 \sec ^{6}(\eta) \xi_{1}^{2} \xi_{2}^{2} \xi_{4}^{6} a^{\prime}(t)^{4}}{-}$ $\alpha^{12} Q_{\alpha, 4}^{8}-\frac{\alpha^{12} Q_{\alpha, 4}^{8}}{\alpha^{10} Q_{\alpha, 4}^{8}}-\frac{\alpha a^{4}}{\alpha^{10} Q_{\alpha, 4}^{8}}-\frac{215040}{\alpha^{12} Q_{\alpha, 4}^{8}}$ $\frac{322560 \sec ^{4}(\eta) \xi_{1}^{2} \xi_{2}^{4} \xi_{4}^{4} a^{\prime}(t)^{4}}{{ }^{12} Q_{\alpha}^{8}}-\frac{241920 \sec ^{4}(\eta) \xi_{1}^{4} \xi_{2}^{2} \xi_{4}^{4} a^{\prime}(t)^{4}}{\alpha^{10} Q_{\alpha}^{8}}-\frac{215040 \sec ^{2}(\eta) \xi_{1}^{2} \xi_{2}^{6} \xi_{4}^{2} a^{\prime}(t)^{4}}{\alpha^{12} Q^{8}}-\frac{241920 \sec ^{2}(\eta) \xi_{1}^{4} \xi_{2}^{4} \xi_{4}^{2} a^{\prime}(t)^{4}}{\alpha^{10} Q_{\alpha, 4}}+$ $\alpha^{12} Q_{\alpha, 4}^{8}-\frac{21020}{\alpha^{10} Q_{\alpha, 4}^{8}}-\frac{24120{ }^{12} Q_{\alpha, 4}^{8}}{\alpha^{10} Q_{\alpha, 4}^{8}}$ $\frac{107520 \xi_{1}^{4} \xi_{2}^{8} a^{\prime}(t)^{4}}{1^{12} Q^{9}}+\frac{107520 \sec ^{8}(\eta) \xi_{1}^{4} \xi_{4}^{8} a^{\prime}(t)^{4}}{\alpha^{12} Q^{9}}+\frac{430080 \sec ^{6}(\eta) \xi_{1}^{4} \xi_{2}^{2} \xi_{4}^{6} a^{\prime}(t)^{4}}{\alpha^{12} Q^{9}}+\frac{645120 \sec ^{4}(\eta) \xi_{1}^{4} \xi_{2}^{4} \xi_{4}^{4} a^{\prime}(t)^{4}}{\alpha^{12} Q^{9}}+$ $\frac{430080 \sec ^{2}(\eta) \xi_{1}^{4} \xi_{2}^{6} \xi_{4}^{2} a^{\prime}(t)^{4}}{\alpha^{12} Q^{9}}-\frac{104 \cot (2 \eta) \sec ^{2}(\eta) \xi_{4}^{2} \tan ^{3}(\eta)}{\alpha^{6} Q^{4}}+\frac{928 \cot (2 \eta) \sec ^{4}(\eta) \xi_{4}^{4} \tan ^{3}(\eta)}{\alpha^{8} Q^{5}}+\frac{1600 \cot (2 \eta) \sec ^{2}(\eta) \xi_{2}^{2} \xi_{4}^{2} \tan ^{3}(\eta)}{\alpha^{8} Q^{5}}-$ $\alpha^{12} Q_{\alpha, 4}^{9} Q_{\alpha, 4}^{4} \alpha^{8} Q_{\alpha, 4}^{5} \quad+\frac{\alpha^{8} Q_{\alpha, 4}^{5}}{2}$ $\frac{1280 \cot (2 \eta) \sec ^{6}(\eta) \xi_{4}^{6} \tan ^{3}(\eta)}{\alpha^{10} Q^{6}}-\frac{16960 \cot (2 \eta) \sec ^{4}(\eta) \xi_{2}^{2} \xi_{4}^{4} \tan ^{3}(\eta)}{\alpha^{10} Q^{6}}-\frac{2560 \cot (2 \eta) \sec ^{2}(\eta) \xi_{2}^{4} \xi_{4}^{2} \tan ^{3}(\eta)}{\alpha^{10} Q^{6}}+$ $\frac{26880 \cot (2 \eta) \sec ^{6}(\eta) \xi_{2}^{2} \xi_{4}^{6} \tan ^{3}(\eta)}{\alpha^{12} Q^{7}}+\frac{30720 \cot (2 \eta) \sec ^{4}(\eta) \xi_{2}^{4} \xi_{4}^{4} \tan ^{3}(\eta)}{\alpha^{12} Q^{7}}-\frac{53760 \cot (2 \eta) \sec ^{6}(\eta) \xi_{2}^{4} \xi_{4}^{6} \tan ^{3}(\eta)}{\alpha^{14} Q^{8}}-\frac{4 \sec ^{2}(\eta) \tan ^{2}(\eta)}{\alpha^{4} Q^{3}}+$ $\frac{16 \sec ^{2}(\eta) \xi_{2}^{2} \tan ^{2}(\eta)}{\alpha^{6} Q^{4}}-\frac{251 \sec ^{4}(\eta) \xi_{4}^{2} \tan ^{2}(\eta)}{\alpha^{6} Q^{4}}-\frac{64 \cot ^{2}(2 \eta) \sec ^{2}(\eta) \xi_{4}^{2} \tan ^{2}(\eta)}{\alpha^{6} Q^{4}}+\frac{128 \csc ^{2}(2 \eta) \sec ^{2}(\eta) \xi_{4}^{2} \tan ^{2}(\eta)}{\alpha^{6} Q^{4}}+$ $\frac{36 \sec ^{2}(\eta) \xi_{4}^{2} \tan ^{2}(\eta)}{\alpha^{6} Q_{\alpha, 4}^{4}}+\frac{1520 \sec ^{6}(\eta) \xi_{4}^{4} \tan ^{2}(\eta)}{\alpha^{8} Q_{\alpha, 4}^{5}}+\frac{192 \cot ^{2}(2 \eta) \sec ^{4}(\eta) \xi_{4}^{4} \tan ^{2}(\eta)}{\alpha^{8} Q_{\alpha, 4}^{5}}-\frac{384 \csc ^{2}(2 \eta) \sec ^{4}(\eta) \xi_{4}^{4} \tan ^{2}(\eta)}{\alpha^{8} Q_{\alpha, 4}^{5}}-$ 
$\frac{128 \sec ^{4}(\eta) \xi_{4}^{4} \tan ^{2}(\eta)}{\alpha^{8} Q^{5}}+\frac{3648 \sec ^{4}(\eta) \xi_{2}^{2} \xi_{4}^{2} \tan ^{2}(\eta)}{\alpha^{8} Q^{5}}+\frac{1312 \cot ^{2}(2 \eta) \sec ^{2}(\eta) \xi_{2}^{2} \xi_{4}^{2} \tan ^{2}(\eta)}{\alpha^{8} Q^{5}}-\frac{2624 \csc ^{2}(2 \eta) \sec ^{2}(\eta) \xi_{2}^{2} \xi_{4}^{2} \tan ^{2}(\eta)}{\alpha^{8} Q^{5}}-$ $\frac{256 \sec ^{2}(\eta) \xi_{2}^{2} \xi_{4}^{2} \tan ^{2}(\eta)}{\alpha^{8} Q^{5}}-\frac{1664 \sec ^{8}(\eta) \xi_{4}^{6} \tan ^{2}(\eta)}{\alpha^{10} Q^{6}}-\frac{24592 \sec ^{6}(\eta) \xi_{2}^{2} \xi_{4}^{4} \tan ^{2}(\eta)}{\alpha^{10} Q^{6}}-\frac{5120 \cot ^{2}(2 \eta) \sec ^{4}(\eta) \xi_{2}^{2} \xi_{4}^{4} \tan ^{2}(\eta)}{\alpha^{10} Q_{\alpha, 4}^{6}}+$ $\frac{10240 \csc ^{2}(2 \eta) \sec ^{4}(\eta) \xi_{2}^{2} \xi_{4}^{4} \tan ^{2}(\eta)}{\alpha^{10} Q_{\alpha, 4}^{6}}+\frac{960 \sec ^{4}(\eta) \xi_{2}^{2} \xi_{4}^{4} \tan ^{2}(\eta)}{\alpha^{10} Q_{\alpha}^{6}}-\frac{5632 \sec ^{4}(\eta) \xi_{2}^{4} \xi_{4}^{2} \tan ^{2}(\eta)}{\alpha^{10} Q_{\alpha}^{6}}-\frac{2560 \cot ^{2}(2 \eta) \sec ^{2}(\eta) \xi_{2}^{4} \xi_{4}^{2} \tan ^{2}(\eta)}{\alpha^{10} Q_{\alpha, 4}^{6}}+$ $\frac{5120 \csc ^{2}(2 \eta) \sec ^{2}(\eta) \xi_{2}^{4} \xi_{4}^{2} \tan ^{2}(\eta)}{\alpha^{10} Q_{\alpha, 4}^{6}}+\frac{30080 \sec ^{8}(\eta) \xi_{2}^{2} \xi_{4}^{6} \tan ^{2}(\eta)}{\alpha^{12} Q_{\alpha, 4}^{7}}+\frac{40960 \sec ^{6}(\eta) \xi_{2}^{4} \xi_{4}^{4} \tan ^{2}(\eta)}{\alpha^{12} Q_{\alpha, 4}^{7}}+\frac{11520 \cot ^{2}(2 \eta) \sec ^{4}(\eta) \xi_{2}^{4} \xi_{4}^{4} \tan ^{2}(\eta)}{\alpha^{12} Q_{\alpha, 4}^{7}}-$ $\frac{23040 \csc ^{2}(2 \eta) \sec ^{4}(\eta) \xi_{2}^{4} \xi_{4}^{4} \tan ^{2}(\eta)}{\alpha^{12} Q_{\alpha, 4}^{7}}-\frac{53760 \sec ^{8}(\eta) \xi_{2}^{4} \xi_{4}^{6} \tan ^{2}(\eta)}{\alpha^{14} Q_{\alpha, 4}^{8}}-\frac{87 \sec ^{2}(\eta) \xi_{4}^{2} \tan ^{2}(\eta) a^{\prime}(t)^{2}}{\alpha^{6} Q_{\alpha, 4}^{4}}+\frac{1624 \sec ^{4}(\eta) \xi_{4}^{4} \tan ^{2}(\eta) a^{\prime}(t)^{2}}{\alpha^{8} Q_{\alpha, 4}^{5}}+$ $\frac{216 \sec ^{2}(\eta) \xi_{1}^{2} \xi_{4}^{2} \tan ^{2}(\eta) a^{\prime}(t)^{2}}{\alpha^{6} Q_{\alpha, 4}^{5}}+\frac{2552 \sec ^{2}(\eta) \xi_{2}^{2} \xi_{4}^{2} \tan ^{2}(\eta) a^{\prime}(t)^{2}}{\alpha^{8} Q_{\alpha, 4}^{5}}-\frac{5760 \sec ^{6}(\eta) \xi_{4}^{6} \tan ^{2}(\eta) a^{\prime}(t)^{2}}{\alpha^{10} Q_{\alpha, 4}^{6}}-\frac{9120 \sec ^{4}(\eta) \xi_{1}^{2} \xi_{4}^{4} \tan ^{2}(\eta) a^{\prime}(t)^{2}}{\alpha^{8} Q_{\alpha, 4}^{6}}-$

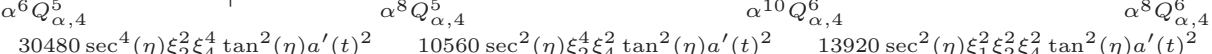
$\frac{30480 \sec ^{4}(\eta) \xi_{2}^{2} \xi_{4}^{4} \tan ^{2}(\eta) a^{\prime}(t)^{2}}{\alpha^{10} Q_{\alpha, 4}^{6}}-\frac{10560 \sec ^{2}(\eta) \xi_{2}^{4} \xi_{4}^{2} \tan ^{2}(\eta) a^{\prime}(t)^{2}}{\alpha^{10} Q_{\alpha, 4}^{6}}-\frac{13920 \sec ^{2}(\eta) \xi_{1}^{2} \xi_{2}^{2} \xi_{4}^{2} \tan ^{2}(\eta) a^{\prime}(t)^{2}}{\alpha^{8} Q_{\alpha, 4}^{6}}+$ $\frac{5120 \sec ^{8}(\eta) \xi_{4}^{8} \tan ^{2}(\eta) a^{\prime}(t)^{2}}{\alpha^{12} Q_{\alpha, 4}^{7}}+\frac{47040 \sec ^{6}(\eta) \xi_{1}^{2} \xi_{4}^{6} \tan ^{2}(\eta) a^{\prime}(t)^{2}}{\alpha^{10} Q_{\alpha, 4}^{7}}+\frac{79040 \sec ^{6}(\eta) \xi_{2}^{2} \xi_{4}^{6} \tan ^{2}(\eta) a^{\prime}(t)^{2}}{\alpha^{12} Q_{\alpha, 4}^{7}}+$ $\frac{84160 \sec ^{4}(\eta) \xi_{2}^{4} \xi_{4}^{4} \tan ^{2}(\eta) a^{\prime}(t)^{2}}{\alpha^{12} Q_{\alpha, 4}^{7}}+\frac{233280 \sec ^{4}(\eta) \xi_{1}^{2} \xi_{2}^{2} \xi_{4}^{4} \tan ^{2}(\eta) a^{\prime}(t)^{2}}{\alpha^{10} Q_{\alpha, 4}^{7}}+\frac{10240 \sec ^{2}(\eta) \xi_{2}^{6} \xi_{4}^{2} \tan ^{2}(\eta) a^{\prime}(t)^{2}}{\alpha^{12} Q_{\alpha, 4}^{7}}+$ $\frac{85440 \sec ^{2}(\eta) \xi_{1}^{2} \xi_{2}^{4} \xi_{4}^{2} \tan ^{2}(\eta) a^{\prime}(t)^{2}}{\alpha^{2}}-\frac{53760 \sec ^{8}(\eta) \xi_{1}^{2} \xi_{4}^{8} \tan ^{2}(\eta) a^{\prime}(t)^{2}}{\alpha^{12} Q_{\alpha}^{8}}-\frac{53760 \sec ^{8}(\eta) \xi_{2}^{2} \xi_{4}^{8} \tan ^{2}(\eta) a^{\prime}(t)^{2}}{-}$ $\begin{gathered}\alpha^{12} Q_{\alpha, 4}^{8} \\ 107520 \sec ^{6}(\eta) \xi_{2}^{4} \xi_{4}^{6} \tan ^{2}(\eta) a^{\prime}(t)^{2}\end{gathered}-\frac{779520 \sec ^{6}(\eta) \xi_{1}^{2} \xi_{2}^{2} \xi_{4}^{6} \tan ^{2}(\eta) a^{\prime}(t)^{2}}{\alpha^{14} Q_{\alpha, 4}^{8}}-\frac{53760 \sec ^{4}(\eta) \xi_{2}^{6} \xi_{4}^{4} \tan ^{2}(\eta) a^{\prime}(t)^{2}}{-}$ $\frac{\alpha^{14} Q_{\alpha, 4}^{8}}{\alpha^{12} Q_{\alpha, 4}^{8}}-\frac{\alpha^{14} Q_{\alpha, 4}^{8}}{\alpha^{2}}$ $\frac{833280 \sec ^{4}(\eta) \xi_{1}^{2} \xi_{2}^{4} \xi_{4}^{4} \tan ^{2}(\eta) a^{\prime}(t)^{2}}{\alpha^{12} Q_{\alpha, 4}^{8}}-\frac{107520 \sec ^{2}(\eta) \xi_{1}^{2} \xi_{2}^{6} \xi_{4}^{2} \tan ^{2}(\eta) a^{\prime}(t)^{2}}{\alpha^{12} Q_{\alpha, 4}^{8}}+\frac{645120 \sec ^{8}(\eta) \xi_{1}^{2} \xi_{2}^{2} \xi_{4}^{8} \tan ^{2}(\eta) a^{\prime}(t)^{2}}{\alpha^{14} Q_{\alpha, 4}^{9}}+$ $\frac{1290240 \sec ^{6}(\eta) \xi_{1}^{2} \xi_{2}^{4} \xi_{4}^{6} \tan ^{2}(\eta) a^{\prime}(t)^{2}}{14}+\frac{645120 \sec ^{4}(\eta) \xi_{1}^{2} \xi_{2}^{6} \xi_{4}^{4} \tan ^{2}(\eta) a^{\prime}(t)^{2}}{1^{14} Q^{9}}-\frac{116 \cot (2 \eta) \sec ^{2}(\eta) \xi_{4}^{2} \tan (\eta) a^{\prime}(t)^{2}}{\alpha^{6} Q_{a}^{4}}+$ $\frac{\alpha^{14} Q_{\alpha, 4}^{9}}{\alpha^{14} Q_{\alpha, 4}^{9}}+\frac{\alpha^{6} Q_{\alpha, 4}^{4}}{\alpha^{4}}$ $\frac{928 \cot (2 \eta) \sec ^{4}(\eta) \xi_{4}^{4} \tan (\eta) a^{\prime}(t)^{2}}{a^{8} Q^{5}}+\frac{288 \cot (2 \eta) \sec ^{2}(\eta) \xi_{1}^{2} \xi_{4}^{2} \tan (\eta) a^{\prime}(t)^{2}}{a^{6} Q_{\alpha}^{5}}+\frac{3712 \cot (2 \eta) \sec ^{2}(\eta) \xi_{2}^{2} \xi_{4}^{2} \tan (\eta) a^{\prime}(t)^{2}}{-}$ $\frac{\alpha^{8} Q_{\alpha, 4}^{5}}{\alpha^{6} Q_{\alpha, 4}^{5}}+\frac{288 \cot (2 \eta) \alpha^{4}}{\alpha^{8} Q_{\alpha, 4}^{5}}$ $\frac{1280 \cot (2 \eta) \sec ^{6}(\eta) \xi_{4}^{6} \tan (\eta) a^{\prime}(t)^{2}}{\alpha^{10} Q^{6}}-\frac{5760 \cot (2 \eta) \sec ^{4}(\eta) \xi_{1}^{2} \xi_{4}^{4} \tan (\eta) a^{\prime}(t)^{2}}{\alpha^{8} Q^{6}}-\frac{16960 \cot (2 \eta) \sec ^{4}(\eta) \xi_{2}^{2} \xi_{4}^{4} \tan (\eta) a^{\prime}(t)^{2}}{\alpha^{10} Q^{6}}-$ $\frac{15680 \cot (2 \eta) \sec ^{2}(\eta) \xi_{2}^{4} \xi_{4}^{2} \tan (\eta) a^{\prime}(t)^{2}}{\alpha^{10} Q^{6}}-\frac{20160 \cot (2 \eta) \sec ^{2}(\eta) \xi_{1}^{2} \xi_{2}^{2} \xi_{4}^{2} \tan (\eta) a^{\prime}(t)^{2}}{\alpha^{8} Q^{6}}+\frac{11520 \cot (2 \eta) \sec ^{6}(\eta) \xi_{1}^{2} \xi_{4}^{6} \tan (\eta) a^{\prime}(t)^{2}}{\alpha^{10} Q^{7}}+$ $\frac{15360 \cot (2 \eta) \sec ^{6}(\eta) \xi_{2}^{2} \xi_{4}^{6} \tan (\eta) a^{\prime}(t)^{2}}{\alpha^{12} Q^{7}}+\frac{30720 \cot (2 \eta) \sec ^{4}(\eta) \xi_{2}^{4} \xi_{4}^{4} \tan (\eta) a^{\prime}(t)^{2}}{\alpha^{12} Q^{7}}+\frac{138240 \cot (2 \eta) \sec ^{4}(\eta) \xi_{1}^{2} \xi_{2}^{2} \xi_{4}^{4} \tan (\eta) a^{\prime}(t)^{2}}{\alpha^{10} Q^{7}}+$ $\frac{15360 \cot (2 \eta) \sec ^{2}(\eta) \xi_{2}^{6} \xi_{4}^{2} \tan (\eta) a^{\prime}(t)^{2}}{\alpha^{12} Q_{\alpha}^{7}}+\frac{126720 \cot (2 \eta) \sec ^{2}(\eta) \xi_{1}^{2} \xi_{2}^{4} \xi_{4}^{2} \tan (\eta) a^{\prime}(t)^{2}}{\alpha^{10} Q_{\alpha, 4}^{7}}-\frac{161280 \cot (2 \eta) \sec ^{6}(\eta) \xi_{1}^{2} \xi_{2}^{2} \xi_{4}^{6} \tan (\eta) a^{\prime}(t)^{2}}{\alpha^{12} Q_{\alpha, 4}^{8}}-$ $\frac{322560 \cot (2 \eta) \sec ^{4}(\eta) \xi_{1}^{2} \xi_{2}^{4} \xi_{4}^{4} \tan (\eta) a^{\prime}(t)^{2}}{\alpha^{12} Q_{\alpha}^{8}}-\frac{161280 \cot (2 \eta) \sec ^{2}(\eta) \xi_{1}^{2} \xi_{2}^{6} \xi_{4}^{2} \tan (\eta) a^{\prime}(t)^{2}}{\alpha^{12} Q_{\alpha, 4}^{8}}-\frac{4 \cot ^{2}(2 \eta) a^{\prime}(t)^{2}}{\alpha^{4} Q^{3}}+\frac{8 \csc ^{2}(2 \eta) a^{\prime}(t)^{2}}{\alpha^{4} Q^{3}}-$ $\frac{3 \sec ^{2}(\eta) a^{\prime}(t)^{2}}{\alpha^{4} Q_{\alpha, 4}^{3}}-\frac{6 a^{\prime}(t)^{2}}{\alpha^{4} Q_{\alpha, 4}^{3}}-\frac{3 \sec ^{2}(\eta) \xi_{1}^{2} a^{\prime}(t)^{2}}{\alpha^{4} Q_{\alpha, 4}^{4}}-\frac{12 \xi_{1}^{2} a^{\prime}(t)^{2}}{\alpha^{4} Q_{\alpha, 4}^{4}}-\frac{116 \cot ^{2}(2 \eta) \xi_{2}^{2} a^{\prime}(t)^{2}}{\alpha^{6} Q_{\alpha, 4}^{4}}+\frac{232 \csc ^{2}(2 \eta) \xi_{2}^{2} a^{\prime}(t)^{2}}{\alpha^{6} Q_{\alpha, 4}^{4}}+\frac{13 \sec ^{2}(\eta) \xi_{2}^{2} a^{\prime}(t)^{2}}{\alpha^{6} Q_{\alpha, 4}^{4}}+$ $\frac{52 \xi_{2}^{2} a^{\prime}(t)^{2}}{\alpha^{6} Q_{\alpha, 4}^{4}}-\frac{45 \sec ^{4}(\eta) \xi_{4}^{2} a^{\prime}(t)^{2}}{\alpha^{6} Q_{\alpha, 4}^{4}}+\frac{52 \sec ^{2}(\eta) \xi_{4}^{2} a^{\prime}(t)^{2}}{\alpha^{6} Q_{\alpha, 4}^{4}}+\frac{928 \cot ^{2}(2 \eta) \xi_{2}^{4} a^{\prime}(t)^{2}}{\alpha^{8} Q_{\alpha, 4}^{5}}-\frac{1856 \csc ^{2}(2 \eta) \xi_{2}^{4} a^{\prime}(t)^{2}}{\alpha^{8} Q_{\alpha, 4}^{5}}-\frac{32 \sec ^{2}(\eta) \xi_{2}^{4} a^{\prime}(t)^{2}}{\alpha^{8} Q_{\alpha, 4}^{5}}-$ $\frac{128 \xi_{2}^{4} a^{\prime}(t)^{2}}{\alpha^{8} Q_{\alpha, 4}^{5}}+\frac{432 \sec ^{6}(\eta) \xi_{4}^{4} a^{\prime}(t)^{2}}{\alpha^{8} Q_{\alpha, 4}^{5}}-\frac{128 \sec ^{4}(\eta) \xi_{4}^{4} a^{\prime}(t)^{2}}{\alpha^{8} Q_{\alpha, 4}^{5}}+\frac{288 \cot ^{2}(2 \eta) \xi_{1}^{2} \xi_{2}^{2} a^{\prime}(t)^{2}}{\alpha^{6} Q_{\alpha, 4}^{5}}-\frac{576 \csc ^{2}(2 \eta) \xi_{1}^{2} \xi_{2}^{2} a^{\prime}(t)^{2}}{\alpha^{6} Q_{\alpha, 4}^{5}}-$ $\frac{48 \sec ^{2}(\eta) \xi_{1}^{2} \xi_{2}^{2} a^{\prime}(t)^{2}}{\alpha^{6} Q_{\alpha, 4}^{5}}-\frac{192 \xi_{1}^{2} \xi_{2}^{2} a^{\prime}(t)^{2}}{\alpha^{6} Q_{\alpha, 4}^{5}}+\frac{96 \sec ^{4}(\eta) \xi_{1}^{2} \xi_{4}^{2} a^{\prime}(t)^{2}}{\alpha^{6} Q_{\alpha, 4}^{5}}-\frac{192 \sec ^{2}(\eta) \xi_{1}^{2} \xi_{4}^{2} a^{\prime}(t)^{2}}{\alpha^{6} Q_{\alpha, 4}^{5}}+\frac{1328 \sec ^{4}(\eta) \xi_{2}^{2} \xi_{4}^{2} a^{\prime}(t)^{2}}{\alpha^{8} Q_{\alpha, 4}^{5}}+$ $\frac{928 \cot ^{2}(2 \eta) \sec ^{2}(\eta) \xi_{2}^{2} \xi_{4}^{2} a^{\prime}(t)^{2}}{\alpha^{8} Q_{\alpha, 4}^{5}}-\frac{1856 \csc ^{2}(2 \eta) \sec ^{2}(\eta) \xi_{2}^{2} \xi_{4}^{2} a^{\prime}(t)^{2}}{\alpha^{8} Q_{\alpha, 4}^{5}}-\frac{256 \sec ^{2}(\eta) \xi_{2}^{2} \xi_{4}^{2} a^{\prime}(t)^{2}}{\alpha^{8} Q_{\alpha, 4}^{5}}+\frac{696 \sec ^{4}(\eta) \xi_{3}^{2} \xi_{4}^{2} a^{\prime}(t)^{2}}{\alpha^{8} Q_{\alpha, 4}^{5}}-$ $\frac{1280 \cot ^{2}(2 \eta) \xi_{2}^{6} a^{\prime}(t)^{2}}{a^{10} Q^{6}}+\frac{2560 \csc ^{2}(2 \eta) \xi_{2}^{6} a^{\prime}(t)^{2}}{\alpha^{10} Q^{6}}-\frac{640 \sec ^{8}(\eta) \xi_{4}^{6} a^{\prime}(t)^{2}}{\alpha^{10} Q^{6}}-\frac{5760 \cot ^{2}(2 \eta) \xi_{1}^{2} \xi_{2}^{4} a^{\prime}(t)^{2}}{\alpha^{8} Q^{6}}+\frac{11520 \csc ^{2}(2 \eta) \xi_{1}^{2} \xi_{2}^{4} a^{\prime}(t)^{2}}{\alpha^{8} Q^{6}}+$ $\alpha^{10} Q_{\alpha, 4}^{6} \quad \alpha^{10} Q_{\alpha, 4}^{6} \alpha^{10} Q_{\alpha, 4}^{6} a^{6} \alpha^{8} Q_{\alpha, 4}^{6}(\eta)$ $\frac{240 \sec ^{2}(\eta) \xi_{1}^{2} \xi_{2}^{4} a^{\prime}(t)^{2}}{8}+\frac{960 \xi_{1}^{2} \xi_{2}^{4} a^{\prime}(t)^{2}}{\alpha^{8} Q_{\alpha, 4}^{6}}-\frac{2640 \sec ^{6}(\eta) \xi_{1}^{2} \xi_{4}^{4} a^{\prime}(t)^{2}}{8}+\frac{960 \sec ^{4}(\eta) \xi_{1}^{\alpha} \xi_{4}^{4} a^{\prime}(t)^{2}}{\alpha^{8} Q_{\alpha, 4}^{6}}-\frac{6080 \sec ^{6}(\eta) \xi_{2}^{2} \xi_{4}^{4} a^{\prime}(t)^{2}}{\alpha^{10} Q_{\alpha}^{6}}-$

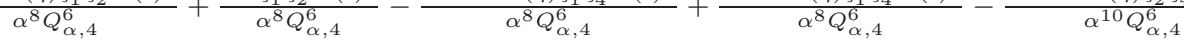
$\frac{1280 \cot ^{2}(2 \eta) \sec ^{4}(\eta) \xi_{2}^{2} \xi_{4}^{4} a^{\prime}(t)^{2}}{\operatorname{loc}^{6}}+\frac{2560 \csc ^{2}(2 \eta) \sec ^{4}(\eta) \xi_{2}^{2} \xi_{4}^{4} a^{\prime}(t)^{2}}{\alpha^{20}}-\frac{6720 \sec ^{6}(\eta) \xi_{3}^{2} \xi_{4}^{4} a^{\prime}(t)^{2}}{\alpha_{\alpha, 4}}-\frac{5440 \sec ^{4}(\eta) \xi_{2}^{4} \xi_{4}^{2} a^{\prime}(t)^{2}}{\alpha_{\alpha, 4}}-$ $\alpha^{10} Q_{\alpha, 4}^{6}+\frac{\alpha^{10} Q_{\alpha, 4}^{6}}{\alpha^{10} Q_{\alpha, 4}^{6}}-\frac{\xi^{2}}{\alpha^{10} Q_{\alpha, 4}^{6}}$ $\frac{2560 \cot ^{2}(2 \eta) \sec ^{2}(\eta) \xi_{2}^{4} \xi_{4}^{2} a^{\prime}(t)^{2}}{\alpha^{10} Q^{6}}+\frac{5120 \csc ^{2}(2 \eta) \sec ^{2}(\eta) \xi_{2}^{4} \xi_{4}^{2} a^{\prime}(t)^{2}}{\alpha^{10} Q^{6}}-\frac{7200 \sec ^{4}(\eta) \xi_{1}^{2} \xi_{2}^{2} \xi_{4}^{2} a^{\prime}(t)^{2}}{\alpha^{8} Q^{6}}-\frac{5760 \cot ^{2}(2 \eta) \sec ^{2}(\eta) \xi_{1}^{2} \xi_{2}^{2} \xi_{4}^{2} a^{\prime}(t)^{2}}{{ }^{8} Q^{6}}+$

$\alpha^{10} Q_{\alpha, 4}^{6}$ $\alpha^{10} Q_{\alpha, 4}^{6} \alpha^{8} Q_{\alpha, 4}^{6}$ $\frac{11520 \csc ^{2}(2 \eta) \sec ^{2}(\eta) \xi_{1}^{2} \xi_{2}^{2} \xi_{4}^{2} a^{\prime}(t)^{2}}{8}+\frac{1920 \sec ^{2}(\eta) \xi_{1}^{2} \xi_{2}^{2} \xi_{4}^{2} a^{\prime}(t)^{2}}{a^{8} Q^{6}}-\frac{4320 \sec ^{4}(\eta) \xi_{1}^{2} \xi_{3}^{2} \xi_{4}^{2} a^{\prime}(t)^{2}}{\alpha^{8} Q^{6}}-\frac{11520 \sec ^{4}(\eta) \xi_{2}^{2} \xi_{3}^{2} \xi_{4}^{2} a^{\prime}(t)^{2}}{\alpha^{10} Q^{6}}+$ $\alpha^{8} Q_{\alpha, 4}^{6} \alpha^{8} Q_{\alpha, 4}^{6} \alpha^{8} Q_{\alpha, 4}^{6}$ $\frac{11520 \cot ^{2}(2 \eta) \xi_{1}^{2} \xi_{2}^{6} a^{\prime}(t)^{2}}{\alpha^{10} Q^{7}}-\frac{23040 \csc ^{2}(2 \eta) \xi_{1}^{2} \xi_{2}^{6} a^{\prime}(t)^{2}}{\alpha^{10} Q^{7}}+\frac{5760 \sec ^{8}(\eta) \xi_{1}^{2} \xi_{4}^{6} a^{\prime}(t)^{2}}{\alpha^{10} Q^{7}}+\frac{5120 \sec ^{8}(\eta) \xi_{2}^{2} \xi_{4}^{6} a^{\prime}(t)^{2}}{\alpha^{12} Q^{7}}+$ $\frac{10240 \sec ^{8}(\eta) \xi_{3}^{2} \xi_{4}^{6} a^{\prime}(t)^{2}}{\alpha^{12} Q_{7}^{7}}+\frac{10240 \sec ^{6}(\eta) \xi_{2}^{4} \xi_{4}^{4} a^{\prime}(t)^{2}}{\alpha^{12} Q^{7}}+\frac{49920 \sec ^{6}(\eta) \xi_{1}^{2} \xi_{2}^{2} \xi_{4}^{4} a^{\prime}(t)^{2}}{\alpha^{10} Q^{7}}+\frac{11520 \cot ^{2}(2 \eta) \sec ^{4}(\eta) \xi_{1}^{2} \xi_{2}^{2} \xi_{4}^{4} a^{\prime}(t)^{2}}{\alpha^{10} Q^{7}}-$ $\alpha^{12} Q_{\alpha, 4}^{7}+\frac{\alpha^{10} Q_{\alpha, 4}^{7}}{\alpha^{12} Q_{\alpha, 4}^{7}} \alpha^{10} Q_{\alpha, 4}^{7}$ $\frac{23040 \csc ^{2}(2 \eta) \sec ^{4}(\eta) \xi_{1}^{2} \xi_{2}^{2} \xi_{4}^{4} a^{\prime}(t)^{2}}{\alpha^{10} Q_{\alpha, 4}^{7}}+\frac{55680 \sec ^{6}(\eta) \xi_{1}^{2} \xi_{3}^{2} \xi_{4}^{4} a^{\prime}(t)^{2}}{\alpha^{10} Q_{\alpha, 4}^{7}}+\frac{89280 \sec ^{6}(\eta) \xi_{2}^{2} \xi_{3}^{2} \xi_{4}^{4} a^{\prime}(t)^{2}}{\alpha^{12} Q_{\alpha, 4}^{7}}+\frac{5120 \sec ^{4}(\eta) \xi_{2}^{6} \xi_{4}^{2} a^{\prime}(t)^{2}}{\alpha^{12} Q_{\alpha, 4}^{7}}+$ $\frac{44160 \sec ^{4}(\eta) \xi_{1}^{2} \xi_{2}^{4} \xi_{4}^{2} a^{\prime}(t)^{2}}{\alpha^{10} Q_{\alpha, 4}^{7}}+\frac{23040 \cot ^{2}(2 \eta) \sec ^{2}(\eta) \xi_{1}^{2} \xi_{2}^{4} \xi_{4}^{2} a^{\prime}(t)^{2}}{\alpha^{10} Q_{\alpha, 4}^{7}}-\frac{46080 \csc ^{2}(2 \eta) \sec ^{2}(\eta) \xi_{1}^{2} \xi_{2}^{4} \xi_{4}^{2} a^{\prime}(t)^{2}}{\alpha^{10} Q_{\alpha, 4}^{7}}+$ 
$\frac{20480 \sec ^{4}(\eta) \xi_{2}^{4} \xi_{3}^{2} \xi_{4}^{2} a^{\prime}(t)^{2}}{\alpha^{12} Q^{7}}+\frac{94080 \sec ^{4}(\eta) \xi_{1}^{2} \xi_{2}^{2} \xi_{3}^{2} \xi_{4}^{2} a^{\prime}(t)^{2}}{\alpha^{10} Q^{7}}-\frac{53760 \sec ^{8}(\eta) \xi_{1}^{2} \xi_{2}^{2} \xi_{4}^{6} a^{\prime}(t)^{2}}{\alpha^{12} Q^{8}}-\frac{107520 \sec ^{8}(\eta) \xi_{1}^{2} \xi_{3}^{2} \xi_{4}^{6} a^{\prime}(t)^{2}}{\alpha^{12} Q^{8}}-$ $\frac{107520 \sec ^{8}(\eta) \xi_{2}^{2} \xi_{3}^{2} \xi_{4}^{6} a^{\prime}(t)^{2}}{\alpha^{14} Q_{\alpha}^{8}}-\frac{107520 \sec ^{6}(\eta) \xi_{1}^{2} \xi_{2}^{4} \xi_{4}^{4} a^{\prime}(t)^{2}}{\alpha^{12} Q_{\alpha}^{8}}-\frac{107520 \sec ^{6}(\eta) \xi_{2}^{4} \xi_{3}^{2} \xi_{4}^{4} a^{\prime}(t)^{2}}{\alpha^{14} Q_{\alpha}^{8}}-\frac{887040 \sec ^{6}(\eta) \xi_{1}^{2} \xi_{2}^{2} \xi_{3}^{2} \xi_{4}^{4} a^{\prime}(t)^{2}}{\alpha^{12} Q_{\alpha, 4}^{8}}-$ $\frac{53760 \sec ^{4}(\eta) \xi_{1}^{2} \xi_{2}^{6} \xi_{4}^{2} a^{\prime}(t)^{2}}{\alpha^{12} Q_{\alpha, 4}^{8}}-\frac{215040 \sec ^{4}(\eta) \xi_{1}^{2} \xi_{2}^{4} \xi_{3}^{2} \xi_{4}^{2} a^{\prime}(t)^{2}}{\alpha^{12} Q_{\alpha, 4}^{8}}+\frac{1290240 \sec ^{8}(\eta) \xi_{1}^{2} \xi_{2}^{2} \xi_{3}^{2} \xi_{4}^{6} a^{\prime}(t)^{2}}{\alpha^{14} Q_{\alpha, 4}^{9}}+\frac{1290240 \sec ^{6}(\eta) \xi_{1}^{2} \xi_{2}^{4} \xi_{3}^{2} \xi_{4}^{4} a^{\prime}(t)^{2}}{\alpha^{14} Q_{\alpha}^{9}}+$ $\frac{9 a^{\prime \prime}(t)^{2}}{\alpha^{2} Q_{\alpha, 4}^{3}}-\frac{108 \xi_{1}^{2} a^{\prime \prime}(t)^{2}}{\alpha^{2} Q_{\alpha, 4}^{4}}+\frac{20 \xi_{2}^{2} a^{\prime \prime}(t)^{2}}{\alpha^{4} Q_{\alpha, 4}^{4}}+\frac{20 \sec ^{2}(\eta) \xi_{4}^{2} a^{\prime \prime}(t)^{2}}{\alpha^{4} Q_{\alpha, 4}^{4}}+\frac{144 \xi_{1}^{4} a^{\prime \prime}(t)^{2}}{\alpha^{2} Q_{\alpha, 4}^{5}}+\frac{112 \xi_{2}^{4} a^{\prime \prime}(t)^{2}}{\alpha^{6} Q_{\alpha, 4}^{5}}+\frac{112 \sec ^{4}(\eta) \xi_{4}^{4} a^{\prime \prime}(t)^{2}}{\alpha^{6} Q_{\alpha, 4}^{5}}-$ $\frac{432 \xi_{1}^{2} \xi_{2}^{2} a^{\prime \prime}(t)^{2}}{\alpha^{4} Q_{\alpha, 4}^{5}}-\frac{432 \sec ^{2}(\eta) \xi_{1}^{2} \xi_{4}^{2} a^{\prime \prime}(t)^{2}}{\alpha^{4} Q_{\alpha, 4}^{5}}+\frac{224 \sec ^{2}(\eta) \xi_{2}^{2} \xi_{4}^{2} a^{\prime \prime}(t)^{2}}{\alpha^{6} Q_{\alpha, 4}^{5}}-\frac{1664 \xi_{1}^{2} \xi_{2}^{4} a^{\prime \prime}(t)^{2}}{\alpha^{6} Q_{\alpha, 4}^{6}}-\frac{1664 \sec ^{4}(\eta) \xi_{1}^{2} \xi_{4}^{4} a^{\prime \prime}(t)^{2}}{\alpha^{6} Q_{\alpha, 4}^{6}}+\frac{768 \xi_{1}^{4} \xi_{2}^{2} a^{\prime \prime}(t)^{2}}{\alpha^{4} Q_{\alpha, 4}^{6}}+$ $\frac{768 \sec ^{2}(\eta) \xi_{1}^{4} \xi_{4}^{2} a^{\prime \prime}(t)^{2}}{a^{4} Q}-\frac{3328 \sec ^{2}(\eta) \xi_{1}^{2} \xi_{2}^{2} \xi_{4}^{2} a^{\prime \prime}(t)^{2}}{a^{6} Q^{2}}+\frac{2560 \xi_{1}^{4} \xi_{2}^{4} a^{\prime \prime}(t)^{2}}{a^{6} Q^{2}}+\frac{2560 \sec ^{4}(\eta) \xi_{1}^{4} \xi_{4}^{4} a^{\prime \prime}(t)^{2}}{2120 \sec ^{2}(\eta) \xi_{1}^{4} \xi_{2}^{2} \xi_{4}^{2} a^{\prime \prime}(t)^{2}}$

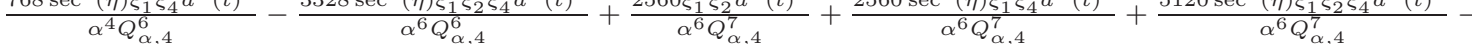
$\frac{4 \cot (2 \eta) \sec ^{2}(\eta) \tan (\eta)}{\alpha^{4} Q_{\alpha, 4}^{3}}+\frac{24 \cot (2 \eta) \sec ^{2}(\eta) \xi_{2}^{2} \tan (\eta)}{\alpha^{6} Q_{\alpha, 4}^{4}}-\frac{220 \cot (2 \eta) \sec ^{4}(\eta) \xi_{4}^{2} \tan (\eta)}{\alpha^{6} Q_{\alpha, 4}^{4}}-\frac{64 \cot (2 \eta) \csc ^{2}(2 \eta) \sec ^{2}(\eta) \xi_{4}^{2} \tan (\eta)}{\alpha^{6} Q_{\alpha, 4}^{4}}+$ $\frac{48 \cot (2 \eta) \sec ^{2}(\eta) \xi_{4}^{2} \tan (\eta)}{\alpha^{6} Q_{\alpha, 4}^{4}}+\frac{512 \cot (2 \eta) \sec ^{6}(\eta) \xi_{4}^{4} \tan (\eta)}{\alpha^{8} Q_{\alpha, 4}^{5}}+\frac{3296 \cot (2 \eta) \sec ^{4}(\eta) \xi_{2}^{2} \xi_{4}^{2} \tan (\eta)}{\alpha^{8} Q_{\alpha, 4}^{5}}+\frac{384 \cot ^{3}(2 \eta) \sec ^{2}(\eta) \xi_{2}^{2} \xi_{4}^{2} \tan (\eta)}{\alpha^{8} Q_{\alpha, 4}^{5}}+$ $\begin{array}{ccc}\alpha^{8} Q_{\alpha, 4}^{5} & \alpha^{8} Q_{\alpha, 4}^{5} & \alpha^{8} Q_{\alpha, 4}^{5} \\ 1536 \cot (2 \eta) \csc ^{2}(2 \eta) \sec ^{2}(\eta) \xi^{2} \xi^{2} \tan (\eta) & 384 \cot (2 \eta) \sec ^{2}(\eta) \xi^{2} \xi^{2} \tan (\eta) & 8960 \cot (2 \eta) \sec ^{6}(\eta) \xi^{2} \xi^{4} \tan ^{2}(\eta)\end{array}$ $\frac{1536 \cot (2 \eta) \csc ^{2}(2 \eta) \sec ^{2}(\eta) \xi_{2}^{2} \xi_{4}^{2} \tan (\eta)}{\alpha^{8} Q_{\alpha, 4}^{5}}-\frac{384 \cot (2 \eta) \sec ^{2}(\eta) \xi_{2}^{2} \xi_{4}^{2} \tan (\eta)}{\alpha^{8} Q_{\alpha, 4}^{5}}-\frac{8960 \cot (2 \eta) \sec ^{6}(\eta) \xi_{2}^{2} \xi_{4}^{4} \tan (\eta)}{\alpha^{10} Q_{\alpha, 4}^{6}}-$ $\frac{5120 \cot (2 \eta) \sec ^{4}(\eta) \xi_{2}^{4} \xi_{4}^{2} \tan (\eta)}{\alpha^{10} Q_{\alpha, 4}^{6}}-\frac{1280 \cot ^{3}(2 \eta) \sec ^{2}(\eta) \xi_{2}^{4} \xi_{4}^{2} \tan (\eta)}{\alpha^{10} Q_{\alpha, 4}^{6}}-\frac{2560 \cot (2 \eta) \csc ^{2}(2 \eta) \sec ^{2}(\eta) \xi_{2}^{4} \xi_{4}^{2} \tan (\eta)}{\alpha^{10} Q_{\alpha, 4}^{6}}+$ $\frac{15360 \cot (2 \eta) \sec ^{6}(\eta) \xi_{2}^{4} \xi_{4}^{4} \tan (\eta)}{\alpha^{2} Q^{7}}+\frac{18 \sec ^{2}(\eta) \xi_{4}^{2} \tan ^{2}(\eta) a^{\prime \prime}(t)}{-336 \sec ^{4}(\eta) \xi_{4}^{4} \tan ^{2}(\eta) a^{\prime \prime}(t)}-\frac{48 \sec ^{2}(\eta) \xi_{1}^{2} \xi_{4}^{2} \tan ^{2}(\eta) a^{\prime \prime}(t)}{\alpha^{5} Q_{\alpha, 4}}-$

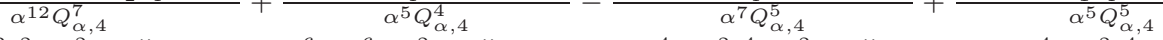
$\frac{528 \sec ^{2}(\eta) \xi_{2}^{2} \xi_{4}^{2} \tan ^{2}(\eta) a^{\prime \prime}(t)}{a^{7}}+\frac{640 \sec ^{6}(\eta) \xi_{4}^{6} \tan ^{2}(\eta) a^{\prime \prime}(t)}{9}+\frac{1600 \sec ^{4}(\eta) \xi_{1}^{2} \xi_{4}^{4} \tan ^{2}(\eta) a^{\prime \prime}(t)}{\alpha^{7} Q^{6}}+\frac{4320 \sec ^{4}(\eta) \xi_{2}^{2} \xi_{4}^{4} \tan ^{2}(\eta) a^{\prime \prime}(t)}{\alpha^{9} Q^{6}}+$ $\alpha^{7} Q_{\alpha, 4}^{5}$ $\frac{1280 \sec ^{2}(\eta) \xi_{2}^{4} \xi_{4}^{2} \tan ^{2}(\eta) a^{\prime \prime}(t)}{9}+\frac{2240 \sec ^{2}(\eta) \xi_{1}^{2} \xi_{2}^{2} \xi_{4}^{2} \tan ^{2}(\eta) a^{\prime \prime}(t)}{a^{7} Q_{6}^{6}}-\frac{5120 \sec ^{6}(\eta) \xi_{1}^{2} \xi_{4}^{6} \tan ^{2}(\eta) a^{\prime \prime}(t)}{Q^{6}}-$ $\frac{5760 \sec ^{6}(\eta) \xi_{2}^{2} \xi_{4}^{6} \tan ^{2}(\eta) a^{\prime \prime}(t)}{\alpha^{\prime 1} Q_{\alpha, 4}^{7}}-\frac{5760 \sec ^{4}(\eta) \xi_{2}^{4} \xi_{4}^{4} \tan ^{2}(\eta) a^{\prime \prime}(t)}{\alpha^{7}}-\frac{28800 \sec ^{4}(\eta) \xi_{1}^{2} \xi_{2}^{2} \xi_{4}^{4} \tan ^{2}(\eta) a^{\prime \prime}(t)}{Q^{7} Q^{7}}$ $\frac{\alpha^{11} Q_{\alpha, 4}^{7}}{\alpha^{11} Q_{\alpha, 4}^{7}}-\frac{2800}{\alpha^{9} Q_{\alpha, 4}^{7}}$ $\frac{10240 \sec ^{2}(\eta) \xi_{1}^{2} \xi_{2}^{4} \xi_{4}^{2} \tan ^{2}(\eta) a^{\prime \prime}(t)}{\alpha^{9} Q^{7}}+\frac{53760 \sec ^{6}(\eta) \xi_{1}^{2} \xi_{2}^{2} \xi_{4}^{6} \tan ^{2}(\eta) a^{\prime \prime}(t)}{\alpha^{11} Q^{8}}+\frac{53760 \sec ^{4}(\eta) \xi_{1}^{2} \xi_{2}^{4} \xi_{4}^{4} \tan ^{2}(\eta) a^{\prime \prime}(t)}{\alpha^{11} Q^{8}}-\frac{9 a^{\prime}(t)^{2} a^{\prime \prime}(t)}{\alpha^{3} Q^{3}}+$ $\frac{108 \xi_{1}^{2} a^{\prime}(t)^{2} a^{\prime \prime}(t)}{\alpha^{3} Q^{4}}-\frac{8 \xi_{2}^{2} a^{\prime}(t)^{2} a^{\prime \prime}(t)}{\alpha^{5} Q^{4}}-\frac{8 \sec ^{2}(\eta) \xi_{4}^{2} a^{\prime}(t)^{2} a^{\prime \prime}(t)}{\alpha^{5} Q^{4}}-\frac{144 \xi_{1}^{4} a^{\prime}(t)^{2} a^{\prime \prime}(t)}{\alpha^{3} Q^{5}}-\frac{688 \xi_{2}^{4} a^{\prime}(t)^{2} a^{\prime \prime}(t)}{\alpha^{7} Q^{5}}-\frac{688 \sec ^{4}(\eta) \xi_{4}^{4} a^{\prime}(t)^{2} a^{\prime \prime}(t)}{\alpha^{7} Q^{5}}+$ $\frac{672 \xi_{1}^{2} \xi_{2}^{2} a^{\prime}(t)^{2} a^{\prime \prime}(t)}{\alpha^{5} Q^{5}}+\frac{672 \sec ^{2}(\eta) \xi_{1}^{2} \xi_{4}^{2} a^{\prime}(t)^{2} a^{\prime \prime}(t)}{\alpha^{5} Q^{5}}-\frac{1376 \sec ^{2}(\eta) \xi_{2}^{2} \xi_{4}^{2} a^{\prime}(t)^{2} a^{\prime \prime}(t)}{\alpha^{7} Q^{5}}+\frac{1664 \xi_{2}^{6} a^{\prime}(t)^{2} a^{\prime \prime}(t)}{\alpha^{9} Q^{6}{ }^{4}}+\frac{1664 \sec ^{6}(\eta) \xi_{4}^{6} a^{\prime}(t)^{2} a^{\prime \prime}(t)}{\alpha^{9} Q^{6}}+$ $\frac{8032 \xi_{1}^{2} \xi_{2}^{4} a^{\prime}(t)^{2} a^{\prime \prime}(t)}{\alpha^{7} Q_{\alpha, 4}^{6}}+\frac{8032 \sec ^{4}(\eta) \xi_{1}^{2} \xi_{4}^{4} a^{\prime}(t)^{2} a^{\prime \prime}(t)}{\alpha^{7} Q_{\alpha, 4}^{6}}+\frac{4992 \sec ^{4}(\eta) \xi_{2}^{2} \xi_{4}^{4} a^{\prime}(t)^{2} a^{\prime \prime}(t)}{\alpha^{9} Q_{\alpha, 4}^{6}}-\frac{1344 \xi_{1}^{4} \xi_{2}^{2} a^{\prime}(t)^{2} a^{\prime \prime}(t)}{\alpha^{5} Q_{\alpha, 4}^{6}}-$

$\frac{1344 \sec ^{2}(\eta) \xi_{1}^{4} \xi_{4}^{2} a^{\prime}(t)^{2} a^{\prime \prime}(t)}{\alpha^{5} Q^{6}}+\frac{4992 \sec ^{2}(\eta) \xi_{2}^{4} \xi_{4}^{2} a^{\prime}(t)^{2} a^{\prime \prime}(t)}{\alpha^{9} Q^{6}{ }^{4}}+\frac{16064 \sec ^{2}(\eta) \xi_{1}^{2} \xi_{2}^{2} \xi_{4}^{2} a^{\prime}(t)^{2} a^{\prime \prime}(t)}{\alpha^{7} Q^{6}}-\frac{30080 \xi_{1}^{2} \xi_{2}^{6} a^{\prime}(t)^{2} a^{\prime \prime}(t)}{\alpha^{9} Q_{\alpha}^{7}}-$ $\frac{30080 \sec ^{6}(\eta) \xi_{1}^{2} \xi_{4}^{6} a^{\prime}(t)^{2} a^{\prime \prime}(t)}{\alpha^{9} Q_{\alpha, 4}^{7}}-\frac{9600 \xi_{1}^{4} \xi_{2}^{4} a^{\prime}(t)^{2} a^{\prime \prime}(t)}{\alpha^{7} Q_{\alpha, 4}^{7}}-\frac{9600 \sec ^{4}(\eta) \xi_{1}^{4} \xi_{4}^{4} a^{\prime}(t)^{2} a^{\prime \prime}(t)}{\alpha^{7} Q_{\alpha, 4}^{7}}-\frac{90240 \sec ^{4}(\eta) \xi_{1}^{2} \xi_{2}^{2} \xi_{4}^{4} a^{\prime}(t)^{2} a^{\prime \prime}(t)}{\alpha^{9} Q_{\alpha, 4}^{7}}-$ $\frac{90240 \sec ^{2}(\eta) \xi_{1}^{2} \xi_{2}^{4} \xi_{4}^{2} a^{\prime}(t)^{2} a^{\prime \prime}(t)}{\alpha^{9} Q_{\alpha}^{7}}-\frac{19200 \sec ^{2}(\eta) \xi_{1}^{4} \xi_{2}^{2} \xi_{4}^{2} a^{\prime}(t)^{2} a^{\prime \prime}(t)}{\alpha^{7} Q_{\alpha, 4}^{7}}+\frac{53760 \xi_{1}^{4} \xi_{2}^{6} a^{\prime}(t)^{2} a^{\prime \prime}(t)}{\alpha^{9} Q_{\alpha, 4}^{8}}+\frac{53760 \sec ^{6}(\eta) \xi_{1}^{4} \xi_{4}^{6} a^{\prime}(t)^{2} a^{\prime \prime}(t)}{\alpha^{9} Q^{8}}+$ $\frac{161280 \sec ^{4}(\eta) \xi_{1}^{4} \xi_{2}^{2} \xi_{4}^{4} a^{\prime}(t)^{2} a^{\prime \prime}(t)}{\alpha^{9} Q_{\alpha, 4}^{8}}+\frac{161280 \sec ^{2}(\eta) \xi_{1}^{4} \xi_{2}^{4} \xi_{4}^{2} a^{\prime}(t)^{2} a^{\prime \prime}(t)}{\alpha^{9} Q_{\alpha, 4}^{8}}+\frac{24 \cot (2 \eta) \sec ^{2}(\eta) \xi_{4}^{2} \tan (\eta) a^{\prime \prime}(t)}{\alpha^{5} Q_{\alpha, 4}^{4}}-$ $\frac{192 \cot (2 \eta) \sec ^{4}(\eta) \xi_{4}^{4} \tan (\eta) a^{\prime \prime}(t)}{\alpha^{7} Q_{\alpha, 4}^{5}}+\frac{64 \cot (2 \eta) \sec ^{2}(\eta) \xi_{1}^{2} \xi_{4}^{2} \tan (\eta) a^{\prime \prime}(t)}{\alpha^{5} Q_{\alpha, 4}^{5}}-\frac{768 \cot (2 \eta) \sec ^{2}(\eta) \xi_{2}^{2} \xi_{4}^{2} \tan (\eta) a^{\prime \prime}(t)}{\alpha^{7} Q_{\alpha, 4}^{5}}+$ $\frac{1280 \cot (2 \eta) \sec ^{4}(\eta) \xi_{1}^{2} \xi_{4}^{4} \tan (\eta) a^{\prime \prime}(t)}{\alpha^{7} Q_{\alpha, 4}^{6}}+\frac{1920 \cot (2 \eta) \sec ^{4}(\eta) \xi_{2}^{2} \xi_{4}^{4} \tan (\eta) a^{\prime \prime}(t)}{\alpha^{9} Q_{\alpha, 4}^{6}}+\frac{1920 \cot (2 \eta) \sec ^{2}(\eta) \xi_{2}^{4} \xi_{4}^{2} \tan (\eta) a^{\prime \prime}(t)}{\alpha^{9} Q_{\alpha, 4}^{6}}+$ $\frac{3200 \cot (2 \eta) \sec ^{2}(\eta) \xi_{1}^{2} \xi_{2}^{2} \xi_{4}^{2} \tan (\eta) a^{\prime \prime}(t)}{-15360 \cot (2 \eta) \sec ^{4}(\eta) \xi_{1}^{2} \xi_{2}^{2} \xi_{4}^{4} \tan (\eta) a^{\prime \prime}(t)}-\frac{15360 \cot (2 \eta) \sec ^{2}(\eta) \xi_{1}^{2} \xi_{2}^{4} \xi_{4}^{2} \tan (\eta) a^{\prime \prime}(t)}{-}$ $\alpha^{7} Q_{\alpha, 4}^{6}$
$\frac{\sec ^{2}(\eta) a^{\prime \prime}(t)}{\alpha^{3} Q^{3}}-\frac{4 a^{\prime \prime}(t)}{\alpha^{3} Q^{3}}+\frac{10 \sec ^{2}(\eta) \xi_{1}^{2} a^{\prime \prime}(t)}{\alpha^{3} Q^{4}}+\frac{40 \xi_{1}^{2} a^{\prime \prime}(t)}{\alpha^{3} Q^{4}}+\frac{24 \cot ^{2}(2 \eta) \xi_{2}^{2} a^{\prime \prime}(t)}{\alpha^{5} Q^{4}}-\frac{48 \csc ^{2}(2 \eta) \xi_{2}^{2} a^{\prime \prime}(t)}{\alpha^{5} Q_{\alpha, 4}^{7}}-\frac{6 \sec ^{2}(\eta) \xi_{2}^{2} a^{\prime \prime}(t)}{\alpha^{5} Q^{4}}-$

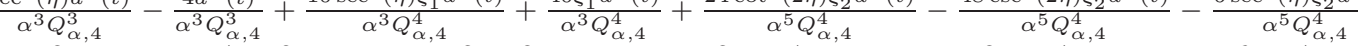
$\frac{24 \xi_{2}^{2} a^{\prime \prime}(t)}{a^{4} Q_{4}^{4}}+\frac{6 \sec ^{4}(\eta) \xi_{4}^{2} a^{\prime \prime}(t)}{0^{4}}-\frac{24 \sec ^{2}(\eta) \xi_{4}^{2} a^{\prime \prime}(t)}{a^{5} Q^{4}}-\frac{192 \cot ^{2}(2 \eta) \xi_{2}^{4} a^{\prime \prime}(t)}{a^{7} Q_{a, 4}}+\frac{384 \csc ^{2}(2 \eta) \xi_{2}^{4} a^{\prime \prime}(t)}{{ }^{7} Q_{5}^{5}}-\frac{96 \sec ^{6}(\eta) \xi_{4}^{4} a^{\prime \prime}(t)}{a^{7} Q^{5}}+$

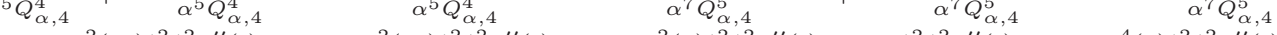
$\frac{64 \cot ^{2}(2 \eta) \xi_{1}^{2} \xi_{2}^{2} a^{\prime \prime}(t)}{\alpha^{5} Q^{5}}-\frac{128 \csc ^{2}(2 \eta) \xi_{1}^{2} \xi_{2}^{2} a^{\prime \prime}(t)}{\alpha^{5} Q^{5}}+\frac{32 \sec ^{2}(\eta) \xi_{1}^{2} \xi_{2}^{2} a^{\prime \prime}(t)}{\alpha^{5} Q^{5}}+\frac{128 \xi_{1}^{2} \xi_{2}^{2} a^{\prime \prime}(t)}{\alpha^{5} Q_{\alpha, 4}^{5}}+\frac{64 \sec ^{4}(\eta) \xi_{1}^{2} \xi_{4}^{2} a^{\prime \prime}(t)}{\alpha^{5} Q^{5}}+$ $\frac{128 \sec ^{2}(\eta) \xi_{1}^{2} \xi_{4}^{2} a^{\prime \prime}(t)}{\alpha^{5} Q_{5}^{5}}-\frac{288 \sec ^{4}(\eta) \xi_{2}^{2} \xi_{4}^{2} a^{\prime \prime}(t)}{{ }^{7} Q_{5}^{5}}-\frac{192 \cot ^{2}(2 \eta) \sec ^{2}(\eta) \xi_{2}^{2} \xi_{4}^{2} a^{\prime \prime}(t)}{{ }^{7} Q^{5}}+\frac{384 \csc ^{2}(2 \eta) \sec ^{2}(\eta) \xi_{2}^{2} \xi_{4}^{2} a^{\prime \prime}(t)}{\alpha^{7} Q_{\alpha, 4}^{5}}-$ $\alpha^{5} Q_{\alpha, 4}^{5}-\frac{\alpha^{7} Q_{\alpha, 4}^{5}}{\alpha^{7} Q_{\alpha, 4}^{5}}-\frac{192 \xi^{2} \xi^{2}}{\alpha^{7} Q_{\alpha, 4}^{5}}$

$\frac{144 \sec ^{4}(\eta) \xi_{3}^{2} \xi_{4}^{2} a^{\prime \prime}(t)}{\alpha^{7} Q^{5}}+\frac{1280 \cot ^{2}(2 \eta) \xi_{1}^{2} \xi_{2}^{4} a^{\prime \prime}(t)}{\alpha^{7} Q^{6}}-\frac{2560 \csc ^{2}(2 \eta) \xi_{1}^{2} \xi_{2}^{4} a^{\prime \prime}(t)}{\alpha^{7} Q^{6}}+\frac{640 \sec ^{6}(\eta) \xi_{1}^{2} \xi_{4}^{4} a^{\prime \prime}(t)}{\alpha^{7} Q^{6}}+\frac{640 \sec ^{6}(\eta) \xi_{2}^{2} \xi_{4}^{4} a^{\prime \prime}(t)}{\alpha^{9} Q^{6}}+$ $\alpha^{7} Q_{\alpha, 4}^{5} \quad \alpha^{7} Q_{\alpha, 4}^{6}$ $\frac{640 \sec ^{6}(\eta) \xi_{3}^{2} \xi_{4}^{4} a^{\prime \prime}(t)}{a^{9} Q^{6}}+\frac{640 \sec ^{4}(\eta) \xi_{2}^{4} \xi_{4}^{2} a^{\prime \prime}(t)}{9^{9} Q^{6}}+\frac{1280 \sec ^{4}(\eta) \xi_{1}^{2} \xi_{2}^{2} \xi_{4}^{2} a^{\prime \prime}(t)}{a^{7} Q^{6}}+\frac{1280 \cot ^{2}(2 \eta) \sec ^{2}(\eta) \xi_{1}^{2} \xi_{2}^{2} \xi_{4}^{2} a^{\prime \prime}(t)}{a^{7} Q^{6}}-$ $\frac{2560 \csc ^{2}(2 \eta) \sec ^{2}(\eta) \xi_{1}^{2} \xi_{2}^{2} \xi_{4}^{2} a^{\prime \prime}(t)}{\alpha^{7} Q^{6}}+\frac{960 \sec ^{4}(\eta) \xi_{1}^{2} \xi_{3}^{2} \xi_{4}^{2} a^{\prime \prime}(t)}{\alpha^{7} Q^{6}}+\frac{1280 \sec ^{4}(\eta) \xi_{2}^{2} \xi_{3}^{2} \xi_{4}^{2} a^{\prime \prime}(t)}{\alpha^{9} Q^{6}}-\frac{5120 \sec ^{6}(\eta) \xi_{1}^{2} \xi_{2}^{2} \xi_{4}^{4} a^{\prime \prime}(t)}{\alpha^{9} Q^{7}}-$ $\frac{5120 \sec ^{6}(\eta) \xi_{1}^{2} \xi_{3}^{2} \xi_{4}^{4} a^{\prime \prime}(t)}{\alpha^{9} Q_{\alpha, 4}^{7}}-\frac{5760 \sec ^{6}(\eta) \xi_{2}^{2} \xi_{3}^{2} \xi_{4}^{4} a^{\prime \prime}(t)}{\alpha^{11} Q_{\alpha, 4}^{7}}-\frac{5120 \sec ^{4}(\eta) \xi_{1}^{2} \xi_{2}^{4} \xi_{4}^{2} a^{\prime \prime}(t)}{\alpha^{9} Q_{\alpha, 4}^{7}}-\frac{10240 \sec ^{4}(\eta) \xi_{1}^{2} \xi_{2}^{2} \xi_{3}^{2} \xi_{4}^{2} a^{\prime \prime}(t)}{\alpha^{9} Q_{\alpha, 4}^{7}}+$ 


$$
\begin{aligned}
& \frac{53760 \sec ^{6}(\eta) \xi_{1}^{2} \xi_{2}^{2} \xi_{3}^{2} \xi_{4}^{4} a^{\prime \prime}(t)}{\alpha^{11} Q_{\alpha, 4}^{8}}+\frac{12 a^{\prime}(t) a^{(3)}(t)}{\alpha^{2} Q_{\alpha, 4}^{3}}-\frac{144 \xi_{1}^{2} a^{\prime}(t) a^{(3)}(t)}{\alpha^{2} Q^{4}}+\frac{36 \xi_{2}^{2} a^{\prime}(t) a^{(3)}(t)}{\alpha^{4} Q^{4}, 4}+\frac{36 \sec ^{2}(\eta) \xi_{4}^{2} a^{\prime}(t) a^{(3)}(t)}{\alpha^{4} Q^{4}{ }^{4}}+ \\
& \frac{192 \xi_{1}^{4} a^{\prime}(t) a^{(3)}(t)}{\alpha^{2} Q_{\alpha, 4}^{5}}+\frac{160 \xi_{2}^{4} a^{\prime}(t) a^{(3)}(t)}{\alpha^{6} Q_{\alpha, 4}^{5}}+\frac{160 \sec ^{4}(\eta) \xi_{4}^{4} a^{\prime}(t) a^{(3)}(t)}{\alpha^{6} Q_{\alpha, 4}^{5}}-\frac{736 \xi_{1}^{2} \xi_{2}^{2} a^{\prime}(t) a^{(3)}(t)}{\alpha^{4} Q_{\alpha, 4}^{5}}-\frac{736 \sec ^{2}(\eta) \xi_{1}^{2} \xi_{4}^{2} a^{\prime}(t) a^{(3)}(t)}{\alpha^{4} Q_{\alpha, 4}^{5}}+ \\
& \frac{320 \sec ^{2}(\eta) \xi_{2}^{2} \xi_{4}^{2} a^{\prime}(t) a^{(3)}(t)}{\alpha^{6} Q^{5}}-\frac{2432 \xi_{1}^{2} \xi_{2}^{4} a^{\prime}(t) a^{(3)}(t)}{\alpha^{6} Q^{6}}-\frac{2432 \sec ^{4}(\eta) \xi_{1}^{2} \xi_{4}^{4} a^{\prime}(t) a^{(3)}(t)}{\alpha^{6} Q^{6}}+\frac{1344 \xi_{1}^{4} \xi_{2}^{2} a^{\prime}(t) a^{(3)}(t)}{\alpha^{4} Q^{6}}+ \\
& \frac{1344 \sec ^{2}(\eta) \xi_{1}^{4} \xi_{4}^{2} a^{\prime}(t) a^{(3)}(t)}{\alpha^{4} Q^{6}}-\frac{4864 \sec ^{2}(\eta) \xi_{1}^{2} \xi_{2}^{2} \xi_{4}^{2} a^{\prime}(t) a^{(3)}(t)}{\alpha^{6} Q^{6}}+\frac{3840 \xi_{1}^{4} \xi_{2}^{4} a^{\prime}(t) a^{(3)}(t)}{\alpha^{6} Q^{7}}+\frac{3840 \sec ^{4}(\eta) \xi_{1}^{4} \xi_{4}^{4} a^{\prime}(t) a^{(3)}(t)}{\alpha^{6} Q^{7}}+ \\
& \frac{7680 \sec ^{2}(\eta) \xi_{1}^{4} \xi_{2}^{2} \xi_{4}^{2} a^{\prime}(t) a^{(3)}(t)}{\alpha^{6} Q_{\alpha, 4}^{7}}+\frac{6 a^{(4)}(t)}{\alpha Q_{\alpha, 4}^{3}}-\frac{72 \xi_{1}^{2} a^{(4)}(t)}{\alpha Q_{\alpha, 4}^{4}}+\frac{8 \xi_{2}^{2} a^{(4)}(t)}{\alpha^{3} Q_{\alpha, 4}^{4}}+\frac{8 \sec ^{2}(\eta) \xi_{4}^{2} a^{(4)}(t)}{\alpha^{3} Q_{\alpha, 4}^{4}}+\frac{96 \xi_{1}^{4} a^{(4)}(t)}{\alpha Q_{\alpha, 4}^{5}}-\frac{96 \xi_{1}^{2} \xi_{2}^{2} a^{(4)}(t)}{\alpha^{3} Q_{\alpha, 4}^{5}}- \\
& \frac{96 \sec ^{2}(\eta) \xi_{1}^{2} \xi_{4}^{2} a^{(4)}(t)}{\alpha^{3} Q_{\alpha, 4}^{5}}+\frac{128 \xi_{1}^{4} \xi_{2}^{2} a^{(4)}(t)}{\alpha^{3} Q_{\alpha, 4}^{6}}+\frac{128 \sec ^{2}(\eta) \xi_{1}^{4} \xi_{4}^{2} a^{(4)}(t)}{\alpha^{3} Q_{\alpha, 4}^{6}}-\frac{7 \sec ^{4}(\eta)}{\alpha^{4} Q_{\alpha, 4}^{3}}+\frac{2 \sec ^{2}(\eta)}{\alpha^{4} Q_{\alpha, 4}^{3}}+\frac{4}{\alpha^{4} Q_{\alpha, 4}^{3}}+\frac{256 \csc ^{4}(2 \eta) \xi_{2}^{2}}{\alpha^{6} Q_{\alpha, 4}^{4}}+ \\
& \frac{8 \sec ^{4}(\eta) \xi_{2}^{2}}{\alpha^{6} Q_{\alpha, 4}^{4}}+\frac{48 \cot ^{2}(2 \eta) \xi_{2}^{2}}{\alpha^{6} Q_{\alpha, 4}^{4}}+\frac{320 \cot ^{2}(2 \eta) \csc ^{2}(2 \eta) \xi_{2}^{2}}{\alpha^{6} Q_{\alpha, 4}^{4}}-\frac{96 \csc ^{2}(2 \eta) \xi_{2}^{2}}{\alpha^{6} Q_{\alpha, 4}^{4}}+\frac{12 \cot ^{2}(2 \eta) \sec ^{2}(\eta) \xi_{2}^{2}}{\alpha^{6} Q_{\alpha, 4}^{4}}-\frac{24 \csc ^{2}(2 \eta) \sec ^{2}(\eta) \xi_{2}^{2}}{\alpha^{6} Q_{\alpha, 4}^{4}}- \\
& \frac{50 \sec ^{6}(\eta) \xi_{4}^{2}}{\alpha^{6} Q^{4}}-\frac{32 \cot ^{2}(2 \eta) \sec ^{4}(\eta) \xi_{4}^{2}}{\alpha^{6} Q^{4}}+\frac{64 \csc ^{2}(2 \eta) \sec ^{4}(\eta) \xi_{4}^{2}}{\alpha^{6} Q^{4}}+\frac{33 \sec ^{4}(\eta) \xi_{4}^{2}}{\alpha^{6} Q^{4}}+\frac{64 \cot ^{4}(2 \eta) \xi_{2}^{4}}{\alpha^{8} Q^{5}}-\frac{256 \csc ^{4}(2 \eta) \xi_{2}^{4}}{\alpha^{8} Q^{5}}- \\
& \frac{768 \cot ^{2}(2 \eta) \csc ^{2}(2 \eta) \xi_{2}^{4}}{\alpha^{8} Q^{5}}+\frac{112 \sec ^{8}(\eta) \xi_{4}^{4}}{\alpha^{8} Q^{5}}-\frac{32 \sec ^{6}(\eta) \xi_{4}^{4}}{\alpha^{8} Q^{5}}+\frac{688 \sec ^{6}(\eta) \xi_{2}^{2} \xi_{4}^{2}}{\alpha^{8} Q^{5}}+\frac{704 \cot ^{2}(2 \eta) \sec ^{4}(\eta) \xi_{2}^{2} \xi_{4}^{2}}{\alpha^{8} Q^{5}}-\frac{1408 \csc ^{2}(2 \eta) \sec ^{4}(\eta) \xi_{2}^{2} \xi_{4}^{2}}{\alpha^{8} Q^{5}}- \\
& \alpha^{8} Q_{\alpha, 4}^{5}+\frac{\alpha^{8} Q_{\alpha, 4}^{5}}{\alpha^{8} Q_{\alpha, 4}^{5}}+\frac{\alpha^{8} Q_{\alpha, 4}^{5}}{\alpha^{8}{ }^{8} Q_{\alpha, 4}^{5}}+\frac{7{ }^{5}}{\alpha^{8} Q_{\alpha, 4}^{5}} \\
& \frac{192 \sec ^{4}(\eta) \xi_{2}^{2} \xi_{4}^{2}}{\alpha^{8} Q_{\alpha, 4}^{5}}-\frac{512 \sec ^{4}(\eta) \xi_{3}^{2} \xi_{4}^{2}}{\alpha^{8} Q_{\alpha, 4}^{5}}-\frac{1664 \sec ^{8}(\eta) \xi_{2}^{2} \xi_{4}^{4}}{\alpha^{10} Q_{\alpha, 4}^{6}}+\frac{240 \sec ^{6}(\eta) \xi_{2}^{2} \xi_{4}^{4}}{\alpha^{10} Q_{\alpha, 4}^{6}}+\frac{6016 \sec ^{6}(\eta) \xi_{3}^{2} \xi_{4}^{4}}{\alpha^{10} Q_{\alpha, 4}^{6}}-\frac{1024 \sec ^{6}(\eta) \xi_{2}^{4} \xi_{4}^{2}}{\alpha^{10} Q_{\alpha, 4}^{6}}- \\
& \frac{1280 \cot ^{2}(2 \eta) \sec ^{4}(\eta) \xi_{2}^{4} \xi_{4}^{2}}{\alpha^{10} Q_{\alpha, 4}^{6}}+\frac{2560 \csc ^{2}(2 \eta) \sec ^{4}(\eta) \xi_{2}^{4} \xi_{4}^{2}}{\alpha^{10} Q_{\alpha, 4}^{6}}+\frac{8768 \sec ^{4}(\eta) \xi_{2}^{2} \xi_{3}^{2} \xi_{4}^{2}}{\alpha^{10} Q_{\alpha, 4}^{6}}-\frac{10240 \sec ^{8}(\eta) \xi_{3}^{2} \xi_{4}^{6}}{\alpha^{12} Q_{\alpha, 4}^{7}}+\frac{2560 \sec ^{8}(\eta) \xi_{2}^{4} \xi_{4}^{4}}{\alpha^{12} Q_{\alpha, 4}^{7}}- \\
& \frac{114560 \sec ^{6}(\eta) \xi_{2}^{2} \xi_{3}^{2} \xi_{4}^{4}}{\alpha^{12} Q_{\alpha, 4}^{7}}-\frac{15360 \sec ^{4}(\eta) \xi_{2}^{4} \xi_{3}^{2} \xi_{4}^{2}}{\alpha^{12} Q_{\alpha, 4}^{7}}+\frac{215040 \sec ^{8}(\eta) \xi_{2}^{2} \xi_{3}^{2} \xi_{4}^{6}}{\alpha^{14} Q_{\alpha, 4}^{8}}+\frac{215040 \sec ^{6}(\eta) \xi_{2}^{4} \xi_{3}^{2} \xi_{4}^{4}}{\alpha^{14} Q_{\alpha, 4}^{8}}-\frac{430080 \sec ^{8}(\eta) \xi_{2}^{4} \xi_{3}^{2} \xi_{4}^{6}}{\alpha^{16} Q_{\alpha, 4}^{9}} .
\end{aligned}
$$

Acknowledgement. The second author acknowledges support from NSF grants DMS-1201512 and PHY-1205440. Part of this work was done at the Perimeter Institute for Theoretical Physics, supported by the Government of Canada through Industry Canada and by the Province of Ontario through the Ministry of Economic Development and Innovation.

\section{REFERENCES}

[1] N. Arkani-Hamed, J. Bourjaily, F. Cachazo, A. Goncharov, A. Postnikov, J. Trnka, Grassmannian Geometry of Scattering Amplitudes, Cambridge University Press, 2016.

[2] S. Bloch, H. Esnault, D. Kreimer, On motives associated to graph polynomials, Comm. Math. Phys. 267 (2006), no. 1, 181-225.

[3] F. Brown, O. Schnetz, A K3 in $\phi^{4}$, Duke Math. J. Vol.161 (2012) no. 10, 1817-1862.

[4] A.H. Chamseddine, A. Connes, Spectral action for Robertson-Walker metrics, J. High Energy Phys. (2012) no. 10, 101, 29 pages

[5] A.H. Chamseddine, A. Connes, The spectral action principle, Comm. Math. Phys. 186 (1997), no. $3,731-750$.

[6] A. Connes, Geometry from the spectral point of view, Lett. Math. Phys. 34 (1995), no. 3, 203238.

[7] A. Connes, M. Marcolli, Renormalization and motivic Galois theory, Int. Math. Res. Not. 2004, no. $76,4073-4091$.

[8] W. Fan, F. Fathizadeh, M. Marcolli, Spectral Action for Bianchi Type-IX Cosmological Models, J. High Energy Phys. 10 (2015) 085.

[9] W. Fan, F. Fathizadeh, M. Marcolli, Modular forms in the spectral action of Bianchi IX gravitational instantons, arXiv:1511.05321

[10] F. Fathizadeh, A. Ghorbanpour, M. Khalkhali, Rationality of spectral action for RobertsonWalker metrics, J. High Energy Phys. (2014) no. 12, 064, f21 pages

[11] J. Golden, A.B. Goncharov, M. Spradlin, C. Vergu, A. Volovich, Motivic Amplitudes and Cluster Coordinates, arXiv:1305.1617

[12] A.B. Goncharov, M. Spradlin, C. Vergu, A. Volovich, Classical Polylogarithms for Amplitudes and Wilson Loops, Phys. Rev. Lett. 105 (2010) no. 15, 151605, 4 pp. 
[13] J.M. Gracia-Bondia, J.C. Varilly, H. Figueroa, Elements of noncommutative geometry, Birkhäuser, 2001.

[14] M. Marcolli, Feynman motives, World Scientific, 2010.

[15] M. Marcolli, Noncommutative Cosmology, World Scientific, to appear.

[16] M. Rost, The motive of a Pfister form, Preprint (1998) www . physik. uni-regensburg. de/ rom03516/motive.html

[17] W. van Suijlekom, Noncommutative Geometry and Particle Physics, Springer, 2014.

[18] A. Vishik, Integral motives of quadrics, Max-Planck-Institut für Mathematik Bonn, Preprint MPI-1998-13, 1-82 (1998).

[19] A. Vishik, Motives of quadrics with applications to the theory of quadratic forms, in "Geometric methods in the algebraic theory of quadratic forms", pp. 25-101, Lecture Notes in Math., Vol.1835, Springer, 2004.

[20] V. Voevodsly, A. Suslin, E.M. Friedlander, Cyles, transfers, and motivic homology theories, Princeton University Press, 2000.

Division of Physics, Mathematics, and Astronomy, California Institute of TechNology, 1200 E California Blvd, Pasadena, CA 91125, USA

E-mail address: farzadf@caltech.edu

E-mail address: matilde@caltech.edu 